\title{
Photosensitivity and Photodarkening of UV-induced Phosphate Glass Fiber Bragg Gratings and Application in Short Fiber Lasers
}

\author{
By \\ Lingyun Xiong, B.Sc, M.Sc, M.Asc

\begin{abstract}
A thesis submitted to
The Faculty of Graduate Studies and Research

in partial fulfilment of the requirements for the degree of

Doctor of Philosophy
\end{abstract} \\ Ottawa-Carleton Institute for Electrical and Computer Engineering \\ Department of Electronics \\ Carleton University \\ Ottawa, Ontario, Canada
}

Copyright (C) Lingyun Xiong, 2014 


\begin{abstract}
\end{abstract}
Phosphate glass fiber is an ideal choice for building compact high gain fiber lasers, as the fiber can host high concentrations of rare earth ions. However, the low photosensitivity of phosphate glass fibers prevents the integration of UV-written intra-core fiber Bragg gratings (FBGs) into the fiber laser cavity. The research presented in this thesis is focused on the investigation of photosensitivity of UV-written Bragg gratings in phosphate glass fibers and their application in direct-written short monolithic fiber lasers.

A systematic investigation of photosensitivity of $\mathrm{Er} / \mathrm{Yb}$ doped and undoped phosphate glass fibers is carried out using irradiation of intense $193 \mathrm{~nm}$ ArF excimer laser light through a phase mask. Thermal annealing experiment results demonstrate that a thermal growth of grating reflectivity of an FBG in phosphate fiber can be obtained upon heating it at temperatures between $100-250{ }^{\circ} \mathrm{C}$. A recipe that can reliably obtain strong FBGs with final index modulation amplitudes between 5 and $10 \times 10^{-5}$ in phosphate fibers through the photo-thermal process is proposed.

An undesirable side effect of FBGs fabrication in a phosphate fiber is discovered that a photodarkening loss of about $1 \mathrm{~dB} / \mathrm{cm}$ at $\mathrm{C}$-band is also induced by $\mathrm{UV}$ irradiation. The photodarkening loss has been identified as the result of formation of a color center at visible band. Photo-bleaching and thermal-bleaching processes are found to be effective in fully erasing the UV-induced photodarkening loss under condition of not diminishing the grating strength.

The application of direct-written strong FBGs in building short monolithic phosphate fiber lasers is investigated. A 3.5 -cm-long single-wavelength singlelongitudinal-mode distributed feedback (DFB) fiber laser has been implemented in the 
$1 \%-2 \% \mathrm{Er} / \mathrm{Yb}$ doped phosphate glass fiber. A laser signal with an output power of 27.5 $\mathrm{mW}$ at a linewidth of $20 \mathrm{MHz}$ is achieved. In addition, a 5-cm-long monolithic dualwavelength single-longitudinal-mode distributed Bragg reflector (DBR) all-phosphate fiber laser is also demonstrated. By exploiting the polarization hole burning effect and the spatial hole burning effect, stable narrow-linewidth dual-wavelength lasing emission with $38 \mathrm{pm}$ wavelength spacing and a total emitted power of $2.8 \mathrm{~mW}$ is obtained from the DBR fiber laser. 


\section{Acknowledgements}

First, I would like to express my deepest gratitude to my supervisor, Professor Jacques Albert. He provided the initial inspiration for this work and gave critical guidance throughout the process. His patience and support made me to keep my courage to overcome the difficulties and accomplish this work.

Furthermore, I am very grateful to Professor Axel Schülzgen from University of Central Florida for supplying phosphate glass fiber samples. Thanks go to Peter Hofmann from Professor Schülzgen's group. His preparing fiber samples and sharing hands-on experiences of handling phosphate fiber are really helpful for me to finish the experimental work.

I would also like to thank my former and current colleagues Albane Laronche, Chengkun Chen, Liyang Shao, Alexander Anderyuk, Yanina Shevchenko, Aliaksandr Bialiayeu, Yang Zhang and Wenjun Zhou for their help and advice.

Finally, I would like to thank my parents and sister for their endless love and support all through my life. Their unconditional love, support and encouragement gave me the strength and determination to reach my academic goal. 
Dedication

To My Parents 


\section{Table of Contents}

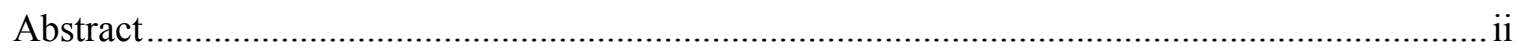

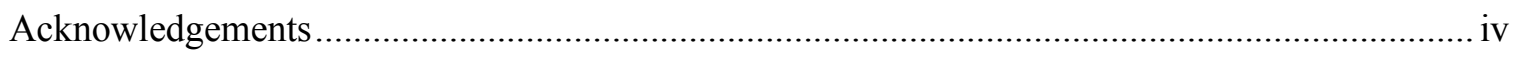

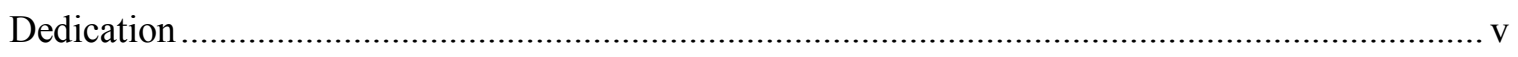

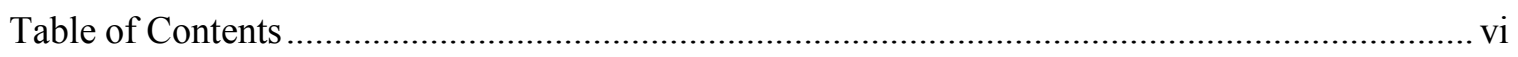

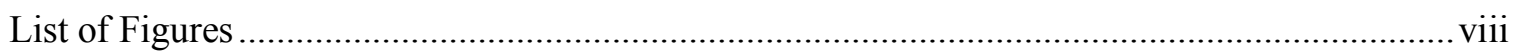

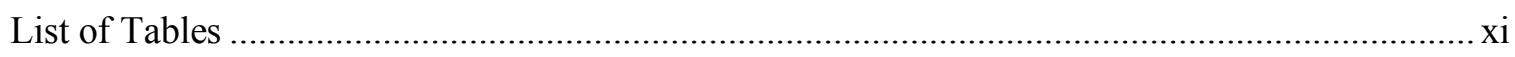

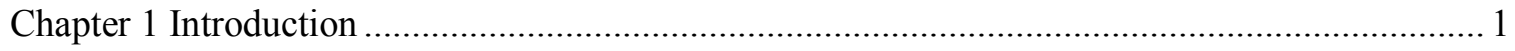

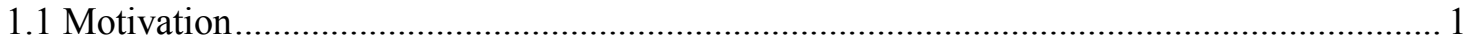

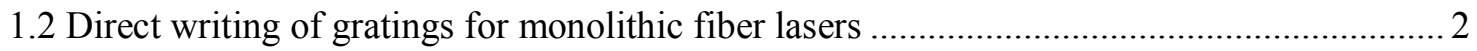

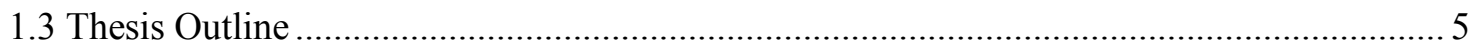

Chapter 2 Background - Theory and Photosensitivity of Fiber Bragg Gratings ........................... 7

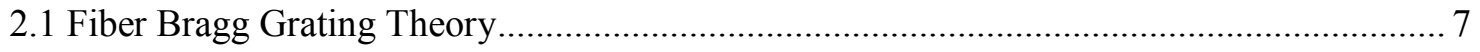

2.1.1 Fiber Bragg gratings spectral characteristics ............................................................ 7

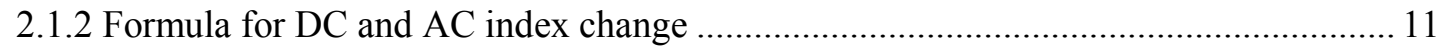

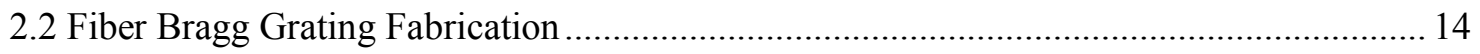

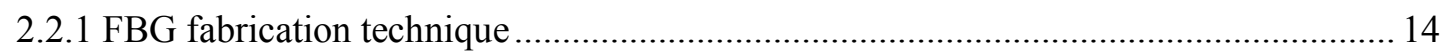

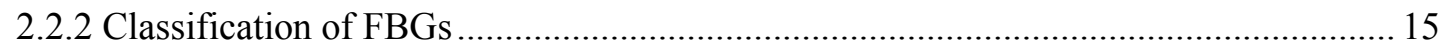

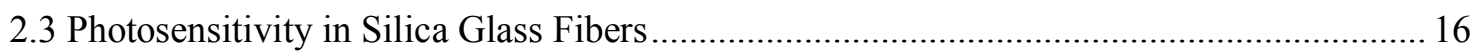

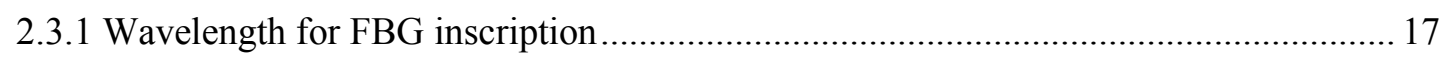

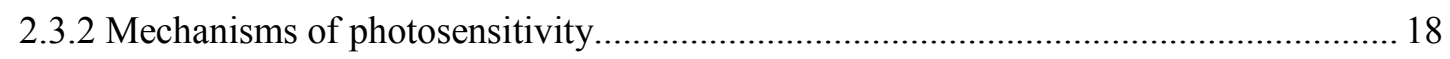

2.4 Photosensitivity in Phosphate Glass Fibers ................................................................... 19

2.4.1 Laser-induced refractive index change in phosphate glasses and fibers ....................... 19

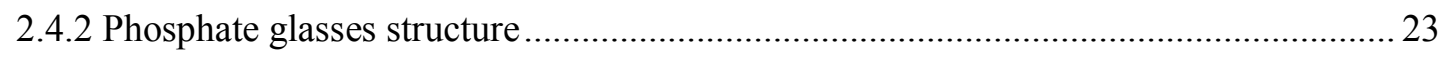

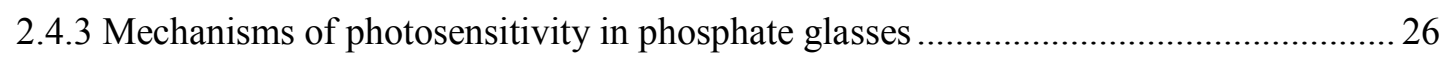

Chapter 3 Photosensitivity and Thermal Stability of FBGs in Phosphate Glass Fibers ................ 30

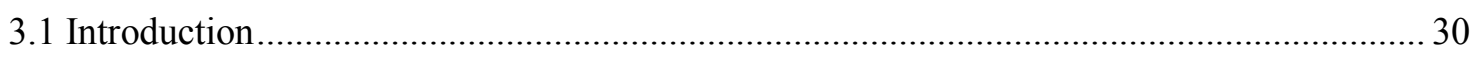

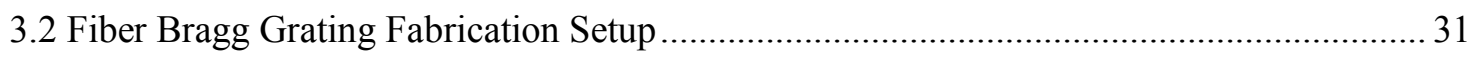

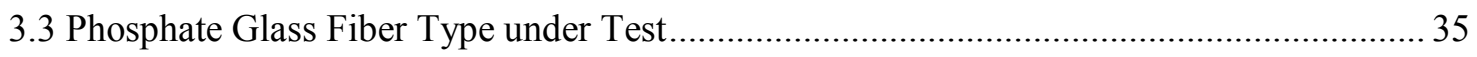

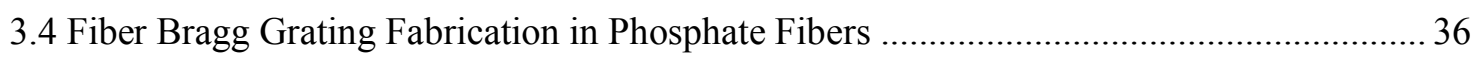

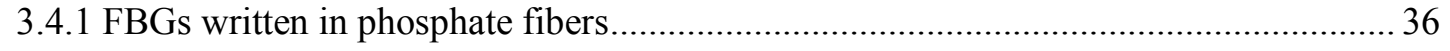

3.4.2 Side effect of FBG inscription: Photodarkening loss ................................................ 38

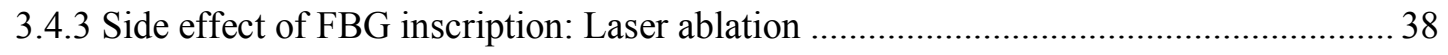


3.5 Photosensitivity and Thermal Stability of FBGs in Phosphate Fibers .............................. 40

3.6 Photosensitivity Mechanism of UV-induced FBGs in Phosphate Fibers ........................... 49

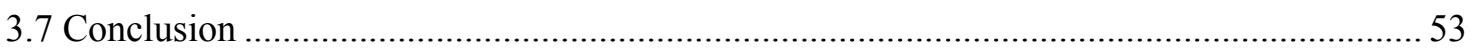

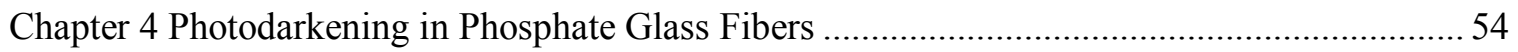

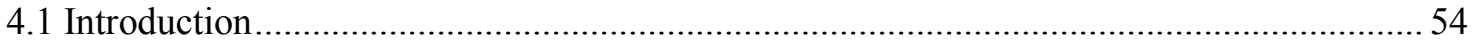

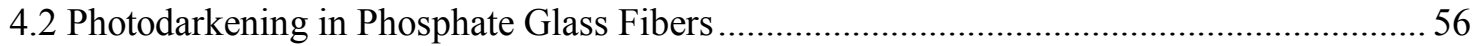

4.3 Photo- and Thermo-bleaching of PD Loss in Phosphate Glass Fibers ................................ 60

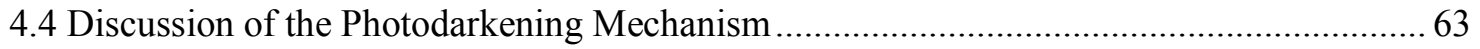

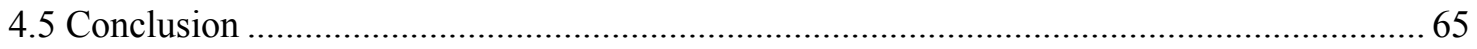

Chapter 5 Single-Wavelength Single-Longitudinal-Mode DFB Phosphate Fiber Laser ............... 66



5.2 Dependence of Laser Output Power on the DFB Grating Reflectivity............................... 73

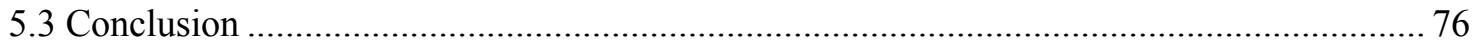

Chapter 6 Dual-wavelength Single-Longitudinal-Mode DBR Phosphate Fiber Laser .................. 77

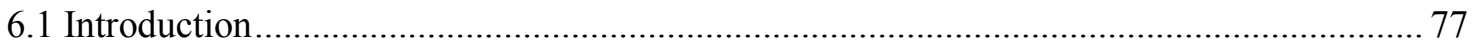

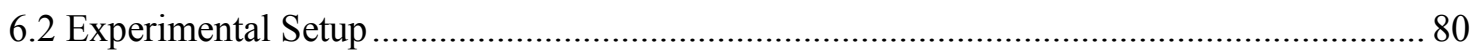

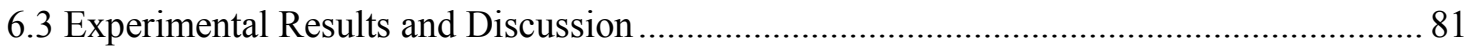

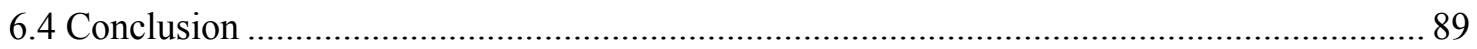

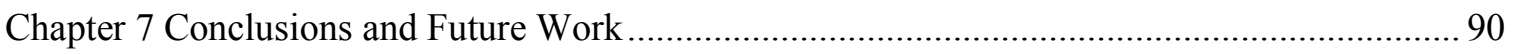

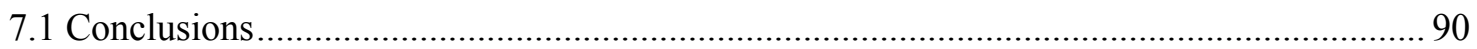

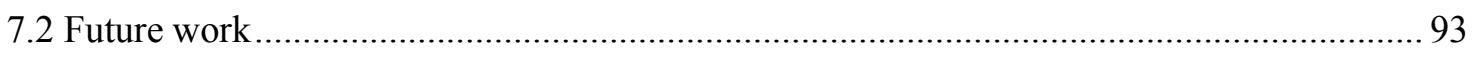

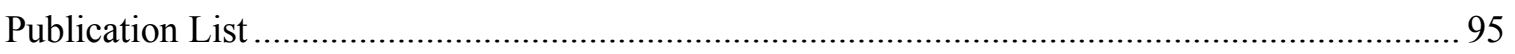

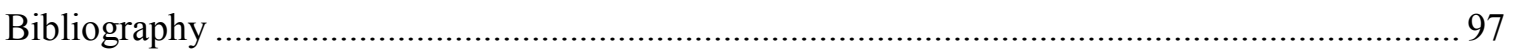




\section{List of Figures}

Figure 2.1 Schematic of a uniform Bragg grating with constant index of modulation amplitude

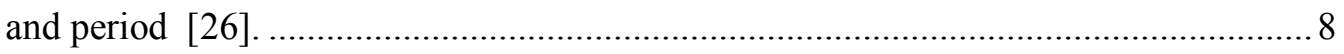

Figure 2.2 Simulated reflection spectrum of a 2-cm-long uniform fiber Bragg grating. ............... 10

Figure 2.3 The refractive index profile of a uniform fiber Bragg grating along the fiber longitudinal axis (a) in the fiber core; and (b) in terms of effective refractive index of

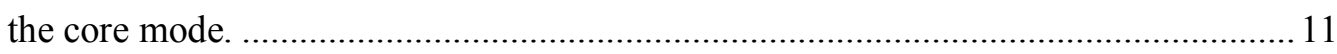

Figure 2.4 Schematic of the phase mask technique for inscribing fiber Bragg gratings............... 15

Figure 2.5 Scheme of photo excitation pathways for different FBG in standard telecommunication

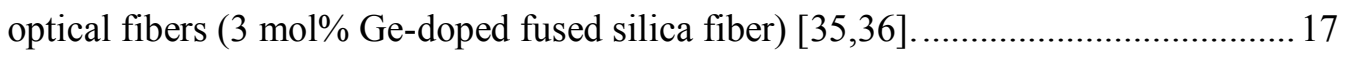

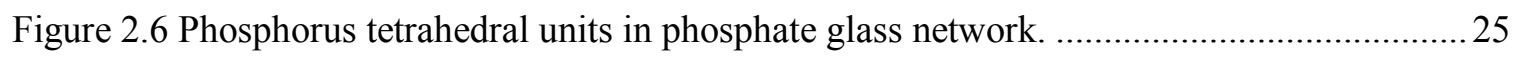

Figure 2.7 UV lamp irradiation-induced color centers in phosphate glass. The irradiation-induced absorption spectra (line) is decomposed into Gaussian peaks (dashed lines) of different

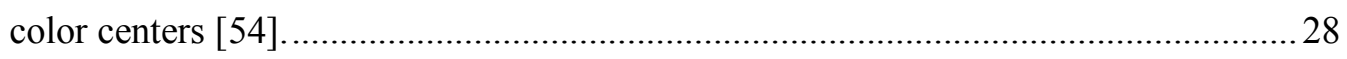

Figure 3.1 Schematic of $193 \mathrm{~nm}$ excimer laser fiber Bragg grating fabrication system................ 32

Figure 3.2 Experimental setup for in-situ measurement of transmission spectra of FBGs in

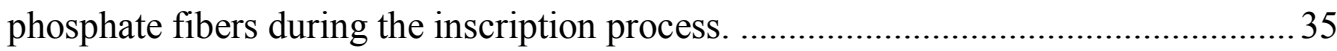

Figure 3.3 Transmission spectra of 2-cm-long UV-induced FBGs in passive and active phosphate fibers.

Figure 3.4 Microscope images of UV-induced damages on the phosphate fiber cladding surface for (a) one end and (b) a middle section of a sample FBG1 in Fiber A; (c) one end and (d) a middle section of a sample FBG2 in Fiber P; (e) cross-section of the cleaved FBG2.

Figure 3.5 Refractive index changes during fabrication of $2 \mathrm{~cm}$-long gratings in passive and active phosphate fibers: (a) $\Delta \mathrm{n}_{\text {avg }}$ in passive fibers (samples P1-P8), (b) $\Delta \mathrm{n}_{\text {avg }}$ in active fibers (samples A1-A4), (c) $\Delta \mathrm{n}_{\text {mod }}$ in passive fibers and (d) $\Delta \mathrm{n}_{\bmod }$ in active fibers. Green solid and dash lines are fitted stretched exponential curves and their respective $95 \%$ prediction bands.

Figure 3.6 Thermal evolution of the transmission notch depths for gratings in passive (P1, P6, P8) and active (A1, A4) phosphate fibers during step-wise annealing between $100-300$ ${ }^{\circ} \mathrm{C}$, in $50{ }^{\circ} \mathrm{C}$ steps for 9-11 days at each step and return to room temperature between steps. See text for further details. 
Figure 3.7 Evolution of transmission spectra of grating P1 measured at room temperature, starting from the UV induced seed and after each thermal annealing step.

Figure 3.8 Evolution of transmission spectra of grating A1 measured at room temperature, starting from the UV induced seed and after each thermal annealing step. .46

Figure 3.9 Thermally induced decay of the average refractive index change $\Delta \mathrm{n}_{\text {avg }}$ relative to its initial value for gratings in passive and active phosphate glass fibers during the annealing described in the caption of Figure 3.6. The initial absolute value of $\Delta \mathrm{n}_{\text {avg }}$ for each sample is included in the legend labels. .47

Figure 3.10 Thermally induced change of refractive index modulation $\Delta \mathrm{n}_{\bmod }$ at the end of the 250

${ }^{\circ} \mathrm{C}$ anneal step versus the UV irradiation time applied to produce the seed gratings...48

Figure 3.11 Phenomenological model of photo-induced index change (black line), thermal annealing (green arrows) and final index changes (red line) for (a) short UV irradiation and (b) long UV irradiation. See text for details.

Figure 4.1 Experimental setup for in-situ characterization of photodarkening loss in phosphate fibers

Figure 4.2 Transmission spectra of photodarkened phosphate fiber samples after $193 \mathrm{~nm}$ irradiation (Samples A-1, P-1, and AP-1 are measured with OSA1, and Samples A-2, P-2, and AP-2 are with OSA2). The inset shows the transmitted intensity spectra of a sample of Fiber A before and after UV irradiation. .58

Figure 4.3 Photodarkening loss spectrum of Fiber A upon 100 pulses exposure, and the Gaussian fitting.

Figure 4.4. Transmission spectrum of the photodarkened Fiber A before, during ( 9 min) and after 40 mins of photo-bleaching.

Figure 4.5 The evolution of photodarkening loss at $670 \mathrm{~nm}$ for phosphate fiber samples during the photodarkening and photo-bleaching processes.

Figure 4.6 The evolution of the average photodarkening loss across C-band of Fiber A during the thermal bleaching. The inset shows the VIS-NIR transmission spectra (normalized by the transmission of the prestine sample) of a fiber sample before and after thermal bleaching. The actual temperature profile is also shown. 63

Figure 5.1 Schematic of the single-wavelength DFB phosphate fiber laser.

Figure 5.2 Experimentally measured (solid line) and simulated (dashed line) transmission spectra of the DFB grating in the Er/Yb co-doped phosphate fiber. .68

Figure 5.3 Optical spectra of the single-wavelength DFB phosphate fiber laser pumped at different launched pump power: 132, 200 and $500 \mathrm{~mW}$. 
Figure 5.4 Output power as a function of launched pump power (bottom axis) and absorbed pump power (top axis) for the DFB fiber laser.

Figure 5.5 Fluctuations of both the lasing wavelength and peak power for the DFB fiber laser

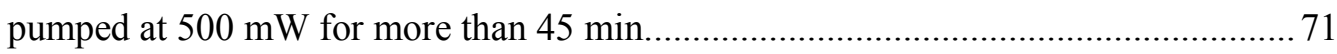

Figure 5.6 Heterodyne spectrum of the single-wavelength DFB fiber laser................................ 73

Figure 5.7 Transmission of the DFB gratings in the Er/Yb doped phosphate fiber: DFB1, DFB2 and DFB3. .75

Figure 5.8 Output power as a function of launched pump power for the single-wavelength DFB fiber lasers: DFB1, DFB2 and DFB3. .75

Figure 6.1 Schematic of the short dual-wavelength single-longitudinal mode DBR phosphate fiber laser. .80

Figure 6.2 Transmission spectra of the uniform FBG1 and the DBR grating structure in the $\mathrm{Er} / \mathrm{Yb}$ doped phpshate fiber. 82

Figure 6.3 Optical spectra of the dual-wavelength DBR phosphate fiber laser pumped at $350 \mathrm{~mW}$ and $500 \mathrm{~mW}$.

Figure 6.4 (a) Laser output spectra for 16 continuous sweeps taken at 1 min interval; fluctuation of (b) wavelengths and (c) peak output powers for dual- wavelength laser pumped at $500 \mathrm{~mW}$

Figure 6.5 Electrical spectrum of the microwave signal generated by the dual- wavelength DBR phosphate fiber laser.

Figure 6.6 Laser output spectra transmitted through a polarization analyzer at different angles. The inset shows the polarization states of two lasing lines $\left(\lambda_{1}\right.$ and $\lambda_{2}$ : short and long lasing wavelengths)

Figure 6.7 Microscope images of UV-induced damages on the phosphate fiber cladding surface for (a) FBG1 with rotation angle $\theta=0^{\circ}$; (b) FBG2 with rotation angle $\theta=70^{\circ}$; (c) cross-section of a cleaved FBG. 


\section{List of Tables}

Table 2.1 Summary of photosensitivity in phosphate glasses and fibers under different irradiation

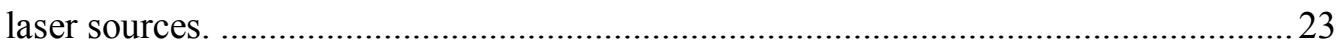

Table 3.1 Parameters for grating inscription in phosphate glass fibers. ....................................... 34

Table 3.2 Details of phosphate fibers used for grating inscription. ............................................. 36

Table 4.1 Details of phosphate fibers used for photodarkening test. ...........................................57 


\section{Chapter 1}

\section{Introduction}

\subsection{Motivation}

Fiber lasers are attractive optical sources for many applications among which are optical fiber communication systems, optical sensing, interferometers and medical instruments.

Silica fibers doped with rare earth ions (e.g. $\mathrm{Er}^{3+}$ and $\mathrm{Yt}^{3+}$ ) are routinely used as the gain medium of fiber lasers [1]. However, the low solubility of silica fiber as a host medium for rare earth ions places a limit on the potential doping concentration of these ions $(<0.1$ mol\%). Further doping of rare earth ions into the silica fiber will lead to cluster formation, which causes concentration quenching and is detrimental to laser performance. This low doping concentration in the silica fiber results in fiber lasers with a cavity of one to tens of meters long to achieve sufficient optical gain. In contrast, the phosphate glasses, which can accommodate higher doping level of rare earth ions (up to several wt\%), will allow one to build a fiber laser with a cavity as short as several centimeters [2-4]. Compared to long fiber lasers, short fiber lasers have the potential to operate at a single longitudinal mode because of the short fiber laser's enlarged longitudinal mode spacing. The single mode operation makes the laser suitable for those applications in which a highly coherent and low-noise light source is necessary. In addition, it will be easy to achieve a small size integrated device if the length of the fiber laser is short. 
The major challenge in developing phosphate fiber lasers is the difficulty of inscribing intracore Bragg gratings as laser resonator mirrors in the phosphate fiber. The phosphate glasses have been found to lack sufficient photosensitivity to inscribe a Bragg grating [5]. In the past, the approach used to create phosphate fiber lasers was to use external resonator mirrors, such as thin-film coating or Bragg gratings written in silica fibers spliced to phosphate fiber [4,6-11], but these kinds of hybrid structures introduce inherent mechanical and optical problems which interfere with laser performance. Different from the aforementioned approaches, the goal of this research is to address the challenge of direct-writing Bragg gratings in phosphate fibers, which should have sufficient reflectivity for developing compact monolithic $\mathrm{Er} / \mathrm{Yb}$ co-doped phosphate fiber lasers. Following our recent previous work $[12,13]$, this thesis will carry out a systematic investigation on the inscription of strong Bragg gratings in phosphate fibers using the standard $193 \mathrm{~nm}$ ultraviolet (UV) irradiation with the phase mask technique. By using this technique, the realization of narrow-linewidth fiber lasers based on distributed feedback (DFB) and distributed Bragg reflector (DBR) structures is achieved in the active phosphate fibers.

\subsection{Direct writing of gratings for monolithic fiber lasers}

Er-doped fiber lasers are one of the most popular rare earth doped fiber lasers because its operation wavelength of $1.5 \mu \mathrm{m}$ band coincides with the third telecommunication window. To increase the pump absorption of the Er-doped fiber, $\mathrm{Yb}$ ions are usually codoped within the fiber. The research interest of direct-writing intracore Bragg gratings into the $\mathrm{Er}$ or $\mathrm{Er} / \mathrm{Yb}$ doped fiber dates back to early 1990s. Short narrow-linewidth 
grating-based fiber lasers were first achieved in Er-doped silica fiber. Both DFB and DBR fiber lasers with cavity lengths of less than $10 \mathrm{~cm}$ were demonstrated [14-21]. Intracore Bragg gratings of several centimeters were inscribed into an active silica fiber by using UV laser irradiation through a phase mask. The short cavity lengths ensured the lasers being able to operate in robust single longitudinal mode without mode hopping. However, as the result of the low doping level of the erbium ions in the silica fiber, the pump absorption was low and the output powers of the fiber lasers were limited to $\mathrm{mW}$ levels. To further utilize the unabsorbed pump power, an additional length of doped fiber can be placed after the laser to amplify the laser signal. This configuration is known as a master oscillator power amplifier (MOPA). An output power of $60 \mathrm{~mW}$ was achieved by using a 19-m long Er-doped fiber [17]. However, the incorporation of the power amplifier generated a high power level of amplified spontaneous emission (ASE) noise, and deteriorated the signal-to-noise ratio. This prevents the MOPA configuration from being used for low-noise sources.

In contrast to silica glasses, phosphate glasses, or even phosphosilicate glasses, can accommodate higher doping levels of erbium and ytterbium ions without forming detrimental ion clusters. The active fibers made from phosphate or phosphosilicate glasses are capable of forming high-power lasers with lengths as short as several centimeters. However, the lack of photosensitivity of both phosphate and phosphosilicate glasses prohibits the direct writing of Bragg gratings in the fibers. A special double-clad fiber design was proposed to incorporate germanium inside the phosphosilicate fiber to improve its photosensitivity [22]. A B/Ge doped silica inner cladding was used to surround the active phosphosilicate fiber core. Strong gratings can be easily fabricated in 
the photosensitive inner cladding, and an output power of $18 \mathrm{~mW}$ has been reached with a cavity length of $3.5 \mathrm{~cm}$. Furthermore, by using a high-reflectivity thin film coating to form the laser cavity with a directly written single grating on the doped fiber, a $1.5-\mathrm{cm}-$ long DBR fiber laser has achieved $60 \mathrm{~mW}$ of output power [23].

The special fiber design to increase the photosensitivity complicates the fabrication process of the active phosphosilicate glass fibers. To circumvent the problem of the low UV photosensitivity, most phosphate fiber lasers resorted to using external resonator mirrors, such as the thin film coating or Bragg gratings fabricated in the silica fiber $[4,6-11]$. The active phosphate glass fibers had thin film coatings deposited on their cleaved ends, or were spliced to the silica fiber on which strong Bragg gratings were written. With two Bragg gratings written in the silica fiber fused section, a short DBR phosphate fiber laser achieved a single-frequency emission with an output power of more than $200 \mathrm{~mW}$ [4]. However, in the above hybrid phosphate-silicate glass devices, both optical losses and mechanical instabilities at the splicing points present inherent challenges due to large differences in thermal properties, such as melting temperature and thermal expansion coefficient, between the different glasses.

Recently, our group successfully demonstrated the direct writing of Bragg gratings in both $\mathrm{Er} / \mathrm{Yb}$ doped and undoped phosphate glass fibers using UV irradiation from an ArF excimer laser at $193 \mathrm{~nm}$ [12,13]. Strong gratings with more than $99 \%$ reflectivity were inscribed in passive phosphate glass fibers. Furthermore, it was found that the grating index modulation can be further strengthened by heating the gratings at temperatures between 100 and $250{ }^{\circ} \mathrm{C}$. This breakthrough on the grating fabrication enabled us to build compact and monolithic DFB phosphate glass fiber lasers [24,25]. By 
using a cladding pumping scheme to couple the high power multimode pump light, a 3.5cm-long DFB phosphate fiber laser achieved output power up to $160 \mathrm{~mW}$. The slope efficiency was only $1.3 \%$ because of the limited pump absorption of the cladding pumping scheme.

In this thesis, we will perform a systematic investigation of the UV-induced Bragg grating fabrication in both $\mathrm{Er} / \mathrm{Yb}$ doped and undoped phosphate glass fibers. The thermal stability of UV-induced gratings in phosphate fibers is studied at elevated temperatures up to $300{ }^{\circ} \mathrm{C}$. The recipe for reliably obtaining strong gratings with high refractive index modulation through the photo-thermal growth process is proposed. Both short DFB and DBR phosphate fiber lasers made with direct writing of Bragg gratings are studied. With a core pumping scheme, the slope efficiency of the fiber laser are improved.

\subsection{Thesis Outline}

The thesis is organized as follows:

Chapter 2 provides an introduction to fiber Bragg gratings (FBGs) in phosphate glass fibers. Fundamental background information regarding fiber Bragg gratings is included. A summary review on the photosensitivity of phosphate glasses and fibers to all different laser irradiations is given. The underlying photosensitivity mechanism of phosphate glasses is explained.

Chapter 3 presents a systematic investigation of FBGs inscription in phosphate glass fibers by using UV irradiation from a $193 \mathrm{~nm}$ excimer laser and the phase mask technique. Photosensitivity and thermal stability of Bragg gratings written in both $\mathrm{Er} / \mathrm{Yb}$ 
doped and undoped phosphate fibers are studied. A recipe that can reliably obtain strong Bragg gratings in phosphate fibers through the photo-thermal process is proposed. Side effects of UV irradiations for grating fabrication in phosphate fibers are also addressed.

Chapter 4 explores the photodarkening (PD) effect in phosphate glass fibers induced by UV irradiation, which is an undesired side effect of UV-induced FBGs fabrication in phosphate fibers. The origin of photodarkening effect in phosphate fibers is attributed to a type of phosphorus-related color center by measuring the PD-induced absorption spectra from the visible (VIS) to near-ultraviolet (NIR) band. Both photobleaching and thermal-bleaching processes are found effective in fully erasing the UVinduced PD loss.

Chapters 5 and 6 demonstrate the application of direct-writing FBGs in $\mathrm{Er} / \mathrm{Yb}$ doped phosphate fibers for constructing short monolithic fiber lasers. A $3.5-\mathrm{cm}-$ long single-wavelength narrow-linewidth DFB phosphate fiber laser is implemented in Chapter 5, while Chapter 6 presents a 5-cm-long dual-wavelength single-longitudinalmode DBR phosphate fiber laser.

Conclusions and possible future work are provided in Chapter 7. 


\section{Chapter 2}

\section{Background - Theory and Photosensitivity of}

\section{Fiber Bragg Gratings}

\subsection{Fiber Bragg Grating Theory}

\subsubsection{Fiber Bragg gratings spectral characteristics}

A fiber Bragg grating consists of a periodic modulation of the refractive index in the core of a single-mode optical fiber, where the phase fronts are perpendicular to the fiber's longitudinal axis. Due to the refractive index variation inside it, each periodic unit of a fiber Bragg grating could be considered as a mirror (optic thin film) to reflect light guided along the core of an optical fiber. Hence, a fiber Bragg grating, as an entity of numerous successive grating planes, is analogous to a volume hologram grating or a crystal lattice diffracting X-rays, in which Bragg diffraction was first discovered. If the wavelength of incident light satisfies the Bragg condition, the reflected light from each of the subsequent grating planes interferes constructively in the backward direction, and as a result the light of this wavelength is reflected by the fiber Bragg grating. Light at other wavelengths that do not satisfy the Bragg condition will get transmitted through the fiber since the reflected light from each grating plane becomes progressively out of phase and will eventually cancel out. 

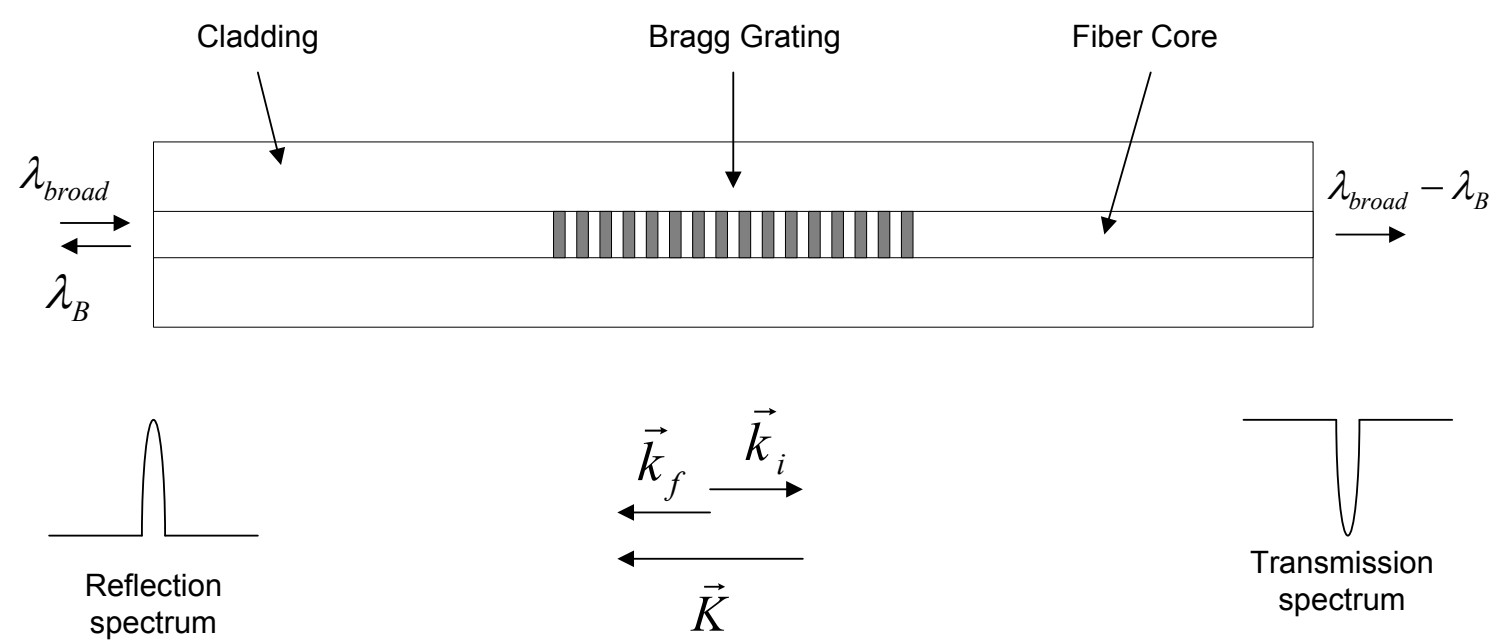

Figure 2.1 Schematic of a uniform Bragg grating with constant index of modulation amplitude and period [26].

Figure 2.1 illustrates a simplest uniform Bragg grating, which has a constant grating period as well as a constant modulation of the refractive index. A fiber Bragg grating functions as an optical filter, which will reflect the light at characteristic wavelength out of a broadband incident light. The characteristic wavelength, called Bragg wavelength, is determined by momentum conservation of the Bragg condition, namely that the summation of the incident wavevector $\overrightarrow{k_{i}}$ and the grating wavevector $\vec{K}$ is equal to the wavevector of the scattered radiation $\overrightarrow{k_{f}}$ :

$$
\overrightarrow{k_{i}}+\vec{K}=\overrightarrow{k_{f}}
$$

Since the incident and reflected light counter propagate inside the fiber, $\overrightarrow{k_{i}}$ and $\overrightarrow{k_{f}}$ should be the propagating constants of fiber modes for forward and backward fields, which have the same magnitude of $2 \pi n_{e f f} / \lambda_{B}$ but in opposite directions. For a first-order Bragg grating, the grating wavevector $\vec{K}$ has a magnitude of $2 \pi / \Lambda$ and a direction normal to the grating planes. With the above conditions, the Bragg condition is simplified as: 


$$
\lambda_{B}=2 n_{e f f} \Lambda
$$

where $\lambda_{B}$ is the Bragg wavelength, $n_{e f f}$ is the effective refractive index for the fiber mode at the Bragg wavelength, and $\Lambda$ is the grating pitch.

In addition to the grating period, the grating's refractive index change is an important parameter controlling the reflectivity and the spectral shape of the grating. Assuming that the refractive index change distributes uniformly across the fiber core and is nonexistent in the outside region, the refractive index profile of a uniform fiber Bragg grating has a sinusoidal modulation form as

$$
n(z)=n_{0}+\Delta n_{\text {mod }} \cos \left(\frac{2 \pi z}{\Lambda}\right)
$$

where $n(z)$ is the refractive index inside the fiber core of a grating, $n_{0}$ is the refractive index of the unperturbed fiber core, $\Delta n_{\bmod }$ is the refractive index modulation amplitude, and $z$ is the distance along the fiber longitudinal axis. According to the coupled mode theory, the reflection properties of a Bragg grating are regarded as light coupling between counter propagating optical fields guided inside the grating and the reflectivity of a uniform fiber Bragg grating is easily calculated from the coupling equations [27,28] as:

$$
R(l, \lambda)=\frac{\kappa^{2} \sinh ^{2}(s l)}{\Delta k^{2} \sinh ^{2}(s l)+s^{2} \cosh ^{2}(s l)}
$$

where $R$ is the power reflectivity of the grating, $l$ is the grating length, $\lambda$ is the reflected wavelength, $\kappa$ is the coupling coefficient, $\Delta k=k-\frac{\pi}{\Lambda}$ is the detuning wavevector (which is the difference between propagation constant at reflected wavelength and that at Bragg wavelength), and $s^{2}=\kappa^{2}-\Delta k^{2}$. Note that the propagation constant $\kappa$ is equal to $2 \pi n_{e f f} / \lambda$, where the effective index $n_{e f f}$ is not the average fiber core refractive index $n_{0}$ in the Eq. (2.3) but can be determined from $n_{0}$. The coupling coefficient $\kappa$ is given by 


$$
\kappa=\frac{\pi \Delta n_{\bmod }}{\lambda} \Gamma
$$

where the confinement factor $\Gamma$ is the fraction of the fiber mode power contained by the fiber core. $\Gamma$ is approximately 0.75 for the fundamental mode of a standard single mode fiber in the wavelength region around $1550 \mathrm{~nm}$. A simulated reflection spectrum of a 2cm-long uniform fiber Bragg grating with $\Delta n_{\text {mod }}$ of $8 \times 10^{-5}$ is shown in Figure 2.2. The maximum reflectivity occurs at the Bragg wavelength, which corresponds to detuning wavevector of zero. Besides the main reflectance peak of the grating, there are several small side lobes situated symmetrically on both sides of the Bragg resonance. For applications that place high demands on the spectral shape of the grating, a technology called apodization can be incorporated into the process of fiber Bragg grating inscription to suppress these undesirable side lobes.

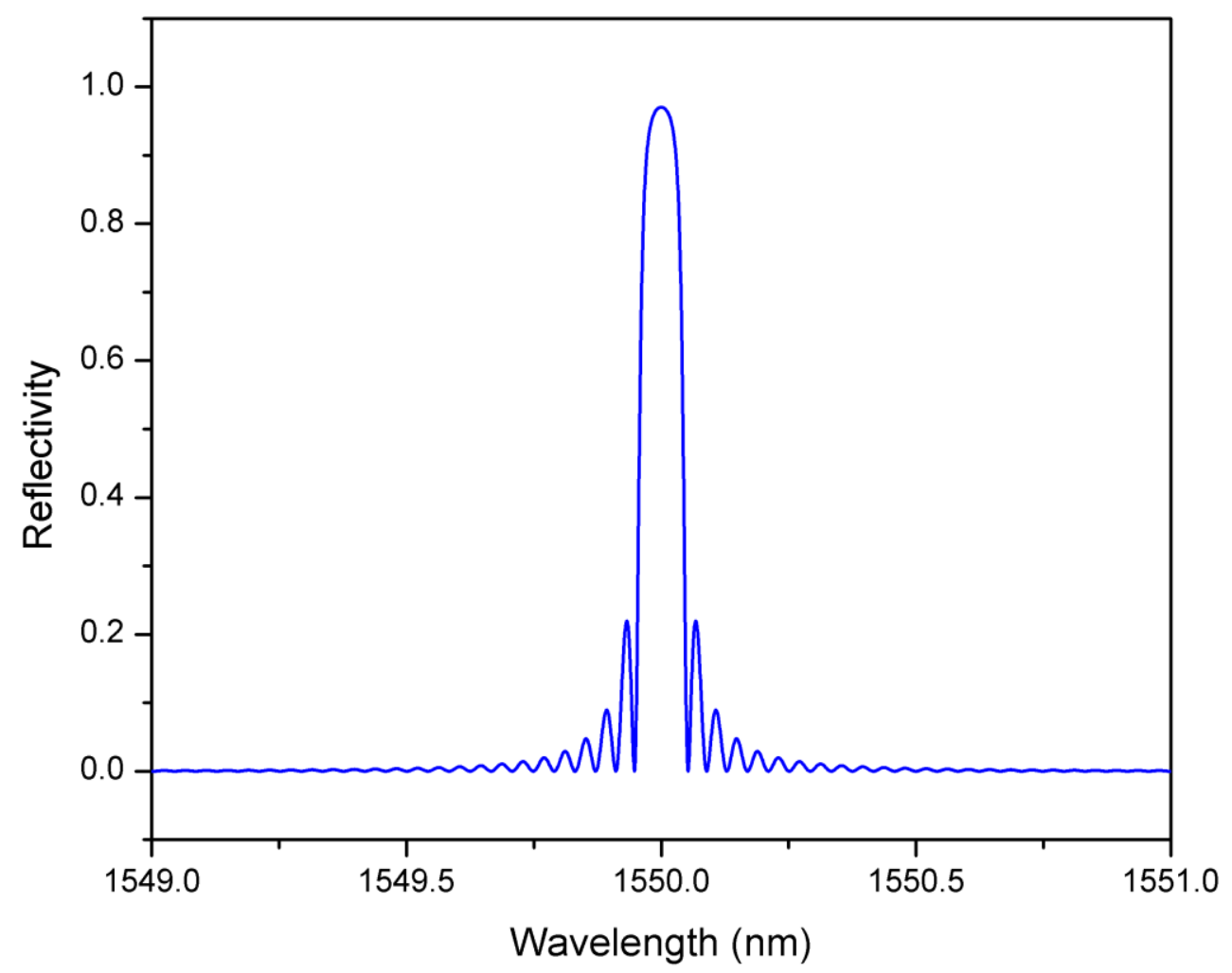

Figure 2.2 Simulated reflection spectrum of a 2-cm-long uniform fiber Bragg grating. 


\subsubsection{Formula for $D C$ and $A C$ index change}

In the above discussion, the index perturbation of a grating is assumed simply as a sinusoidal modulation without any average change imposed on the fiber, which has the form of Eq. (2.3). As a result, the Bragg wavelength $\lambda_{B}$ corresponding to the maximum reflectivity of fiber Bragg grating will be fixed at a single value of $2 n_{\text {eff }} \Lambda$ no matter how strong a grating is formed inside the fiber. However, in practice, it has been observed that the Bragg wavelength of a grating evolves as a function of the irradiation time during the grating fabrication. This shift of Bragg wavelength during the fabrication is caused by the change in the effective refractive index in the grating region, which means that a DC refractive index change is introduced into the grating in addition to an $\mathrm{AC}$ refractive index change.
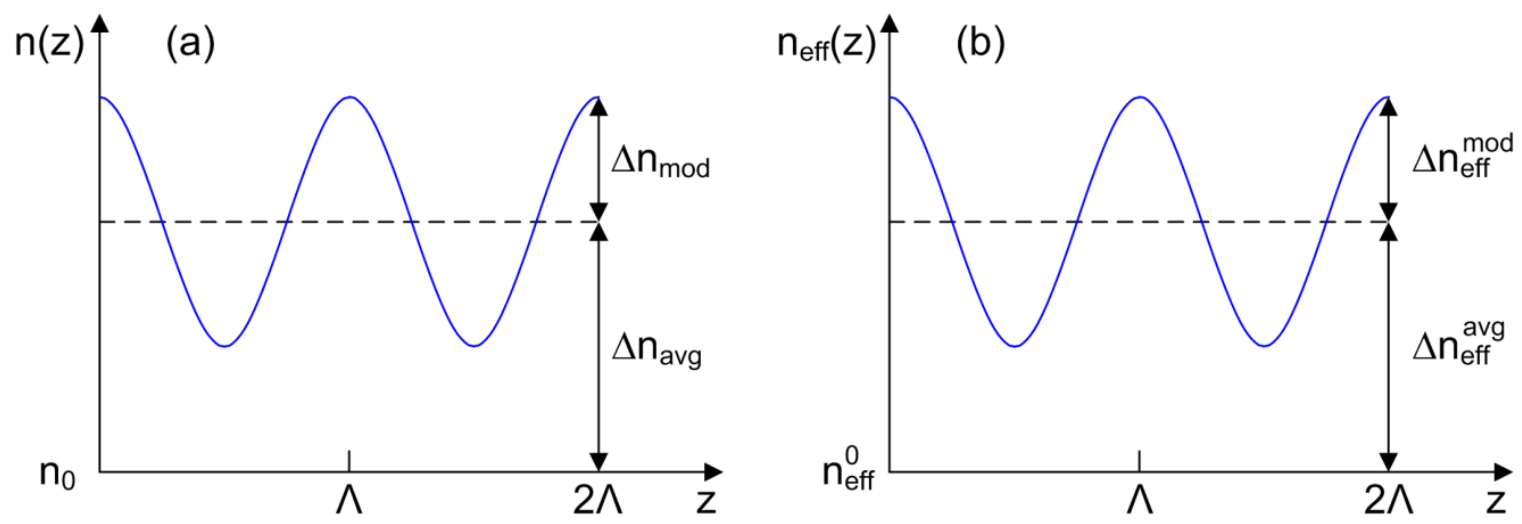

Figure 2.3 The refractive index profile of a uniform fiber Bragg grating along the fiber longitudinal axis (a) in the fiber core; and (b) in terms of effective refractive index of the core mode.

The refractive index profile of a uniform fiber Bragg grating along the fiber longitudinal axis (z-axis) is illustrated in Figure 2.3. In most cases, fiber Bragg gratings are inscribed in the fiber core and not the cladding, because only the fiber core has the photosensitivity to form laser-induced refractive index changes. As seen in Figure 2.3(a), 
the core index profile of a grating that has both DC and AC index change can be written in a more complex form:

$$
n(z)=n_{0}+\Delta n_{\text {avg }}+\Delta n_{\text {mod }} \cos \left(\frac{2 \pi z}{\Lambda}\right)
$$

where $\Delta n_{a v g}$ is the average refractive index change in the fiber core. Other variables in the above equation are the same as those in Eq. (2.3): $n_{0}$ is the refractive index of the unperturbed fiber core, $\Delta n_{m o d}$ is the refractive index modulation amplitude.

Accordingly, the refractive index perturbation of the fiber core results in a similar sinusoidal modulation of the effective index of the fiber guided mode, as seen in Figure 2.3(b). The corresponding profile of the effective index of the guided modes is given by:

$$
n_{e f f}(z)=n_{e f f}^{0}+\Delta n_{e f f}^{a v g}+\Delta n_{e f f}^{m o d} \cos \left(\frac{2 \pi z}{\Lambda}\right)
$$

where $n_{e f f}^{0}$ is the effective refractive index of the unperturbed fiber which has core index of $n_{0}$. Furthermore, $\Delta n_{e f f}^{a v g}$ and $\Delta n_{e f f}^{\text {mod }}$ are the DC and AC components of effective refractive index change respectively.

Assuming that the index perturbation of the grating is uniform across the fiber core and not in the cladding, which is often true due to the lack of photosensitivity of the cladding, the change in the effective refractive index will be approximately proportional to a change in the core index when the latter varies by a small amount (below $1 \times 10^{-3}$ ) [29]. Mathematically, the variables in the Eq. (2.6) and Eq. (2.7) follow the relationship as:

$$
\begin{gathered}
\Delta n_{e f f}^{a v g}=\Delta n_{a v g} \cdot \Gamma \\
\Delta n_{e f f}^{\text {mod }}=\Delta n_{\text {mod }} \cdot \Gamma
\end{gathered}
$$

where $\Gamma$ is the confinement factor of the guided mode. 
As previously mentioned, the shift of Bragg wavelength during the formation of a grating is related to the DC component of the refractive index perturbation. Let $\lambda_{B, 0}$ be the Bragg wavelength at the beginning of the fabrication of the grating written in the pristine fiber, and $\lambda_{B}$ be the Bragg wavelength of the formed grating. According to the Bragg condition, the Bragg wavelengths $\lambda_{B, 0}$ and $\lambda_{B}$ correspond to their own grating's average effective refractive index $n_{e f f}^{0}$ and $n_{e f f}^{0}+\Delta n_{e f f}^{a v g}$, respectively. Consequently, the DC effective index change of a grating $\Delta n_{e f f}^{a v g}$ is given by:

$$
\Delta n_{e f f}^{a v g}=\frac{\lambda_{B}-\lambda_{B, 0}}{2 \Lambda}=\frac{\Delta \lambda_{B}}{2 \Lambda}
$$

where $\Delta \lambda_{B}$ is the Bragg wavelength shift, and $\Lambda$ is the grating pitch. Based on the Eq. (2.8), the DC refractive index change of a grating can be expressed as:

$$
\Delta n_{\text {avg }}=\frac{\Delta \lambda_{B}}{2 \Lambda \Gamma}
$$

Generally, when referring to the index perturbation of a grating, it is more common to use the DC refractive index change in the fiber core rather than the DC effective refractive index change.

The AC component of refractive index change for a FBG can be evaluated from the reflectivity of the grating. The Eq. (2.4) shows that the reflectivity of the grating achieves its maximum at the Bragg wavelength, and the peak reflectivity is given by:

$$
R_{\max }=\tanh ^{2}(\kappa l)
$$

By substituting the expression of the coupling coefficient $\kappa(\mathrm{Eq}(2.5))$ into the above equation, the refractive index modulation amplitude in the fiber core is given by

$$
\Delta n_{\text {mod }}=\frac{\lambda_{B} \tanh ^{-1}\left(\sqrt{R_{\max }}\right)}{\pi \Gamma l}
$$


Its counterpart in terms of effective refractive index is easily expressed as:

$$
\Delta n_{e f f}^{\text {mod }}=\Delta n_{\text {mod }} \cdot \Gamma=\frac{\lambda_{B} \tanh ^{-1}\left(\sqrt{R_{\max }}\right)}{\pi l}
$$

\subsection{Fiber Bragg Grating Fabrication}

\subsubsection{FBG fabrication technique}

The fabrication of a FBG involves introducing a periodic modulation of the refractive index inside the fiber core. This can be achieved by side-exposing the photosensitive fiber to the UV light. The periodic modulation of grating pattern can be formed by using a spatially modulated UV beam, which is a two-beam interference pattern produced by employing either the interferometric technique or the phase mask technique [30-32]. Compared to the interferometric technique, the phase mask technique is a more effective method for FBG fabrication because of the advantages of reducing the complexity of optical setup and providing a robust and stable interference pattern with less sensitivity to mechanical vibration. It is widely used for volume production of FBGs. Therefore, the phase mask technique is selected for FBGs fabrication in this work.

A phase mask, which is a silica plate with periodic etched grooves on one surface, acts as a diffractive grating to diffract the incident light beam in several specific angular directions denoted as diffracted orders $\mathrm{m}(\mathrm{m}$ is an integer, $\mathrm{m}=0, \pm 1, \ldots)$. The interference fringes utilized to imprint the grating are produced by the plus and the minus first-order diffracted beams of the phase mask, which is typically designed to suppress the diffraction efficiency of the zeroth order diffracted beam to less than $3 \%$. A FBG can be manufactured by placing the optical fiber in near contact with the phase mask as shown in Figure 2.4. 
UV laser beam

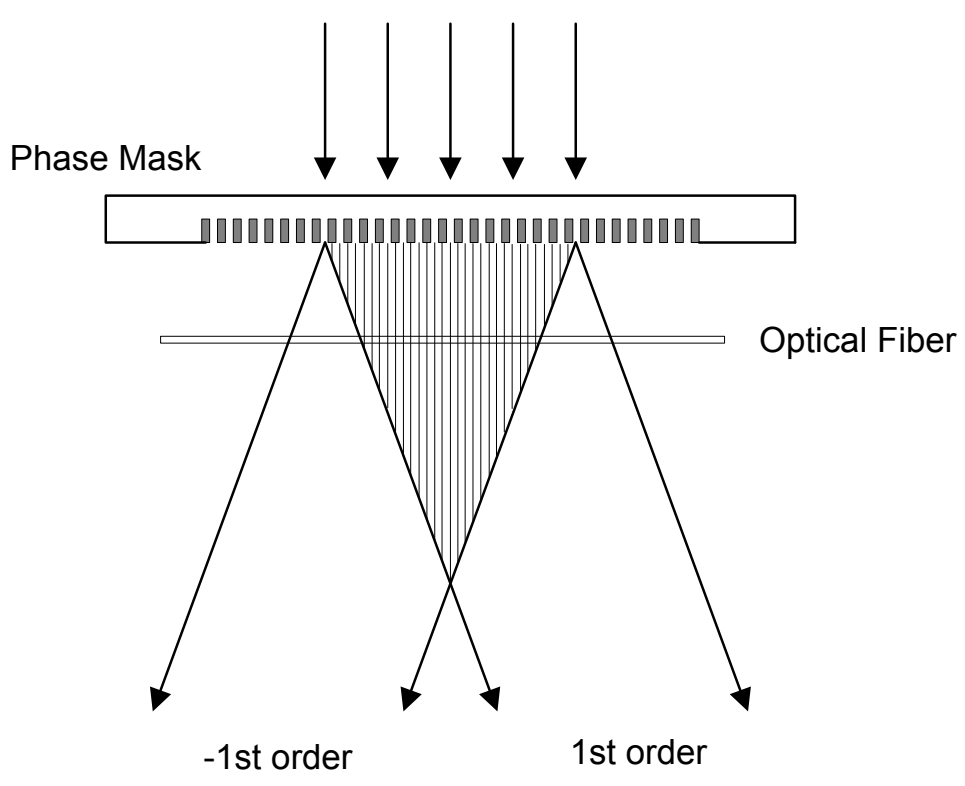

Figure 2.4 Schematic of the phase mask technique for inscribing fiber Bragg gratings.

\subsubsection{Classification of FBGs}

FBGs can be classified into three types according to their growth behavior during the inscription [26,33]. Different types of gratings are usually indicative of the different photosensitivity mechanisms involved in the grating formation.

Type I gratings are generally formed in the photosensitive fibers at modest intensity laser irradiation. A gradual and monotonic growth of positive refractive index change is usually observed. This type of grating is the most common, and it has theoretically expected transmission and reflection spectra.

Type IIA gratings are formed by longer laser irradiation than Type I gratings. Its growth is characterized as an initial growth of positive refractive index change as a Type I grating, which is then erased and subsequently develops as a second grating with negative refractive index change. This grating type is more thermally stable than Type I gratings. 
Type II gratings are formed by irradiation with a single pulse, the intensity of which reaches the damage threshold of the material. The physical damage generated by laser irradiation is localized at the core-cladding interface. As a result of the grating pattern extending to the cladding, strong cladding mode resonances are present in the grating transmission spectrum and leads to large losses on the short wavelength sides of the Bragg wavelength.

\subsection{Photosensitivity in Silica Glass Fibers}

Photosensitivity of an optical fiber refers to the permanent photo-induced refractive index change in the fiber core when exposed to the light irradiation. It is a fundamental property for the inscription of FBGs. Since the discovery of FBGs by Hill et al. in 1978 [34], there has been much effort made to understand the photosensitivity mechanism responsible for the FBGs formation. The mechanism of photo-induced refractive index change is found as a rather complicated process, which is dependent on many factors such as the glass composition, the light source used and the details of irradiation condition. Most of photosensitivity studies were focused on optical fibers made of silica glass, because of its material compatibility with the most widely used standard telecommunication fiber, which is the $3 \mathrm{~mol} \%$ Ge-doped pure silica fiber. A brief review of photosensitivity in silica glass fibers can be used as a good reference for understanding the mechanism in phosphate glass fibers. 


\subsubsection{Wavelength for FBG inscription}

Photosensitivity process of optical fibers requires the absorption of light irradiation by the glass material. Figure 2.5 illustrates photo excitation pathways of several typical laser wavelengths used for FBGs fabrication in silica glass fibers.

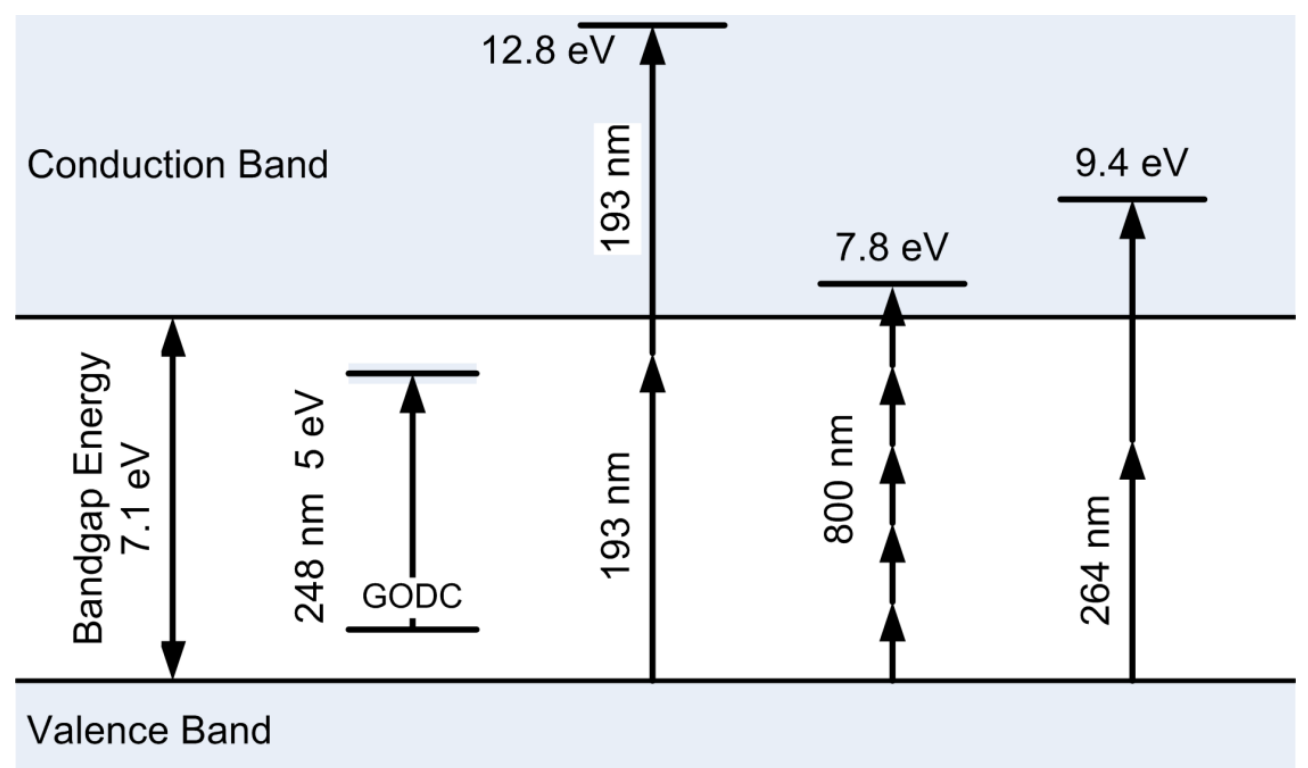

Figure 2.5 Scheme of photo excitation pathways for different FBG in standard telecommunication optical fibers (3 mol\% Ge-doped fused silica fiber) $[35,36]$.

$\mathrm{KrF}(248 \mathrm{~nm})$ and $\operatorname{ArF}(193 \mathrm{~nm})$ excimer lasers are the most commonly used UV irradiation sources for grating fabrication. For these sub-bandgap wavelengths (photon energy lower than $7.1 \mathrm{eV}$ - bandgap energy of silica glass), the pure silica glass is transparent to light at these wavelengths and exhibits no photosensitivity to them. Germanium doping is a conventional photosensitization technique for silica glasses. The $248 \mathrm{~nm}$ irradiation can be absorbed by the defect center - germanium oxygen deficiency center (GODC) through a single-photon absorption process. For the $193 \mathrm{~nm}$ irradiation, in addition to the single-photon absorption process, there is a two-photon absorption process corresponding to photoexcitation of electrons from valence to conduction band of 
silica glasses. The absorption coefficient of the latter process is dependent on the light intensity, and is lower than that of the former process.

The grating inscription with the above UV laser irradiation requires the use of the photosensitive fiber - germanium doped silica fiber. A new approach writing gratings in non-photosensitive fiber becomes possible because of the advent of high-intensity femtosecond lasers. $264 \mathrm{~nm}$ or $800 \mathrm{~nm}$ fs-pulsed laser irradiation can induce refractive index changes based on the non-linear multi-photon absorption process. However, the inscription of high intensity fs-laser induced gratings involves glass damage and makes them as Type II gratings, which have bad quality grating spectra and additional scattering loss.

\subsubsection{Mechanisms of photosensitivity}

Several models have been proposed to explain the photosensitivity effect, but no single model can fully explain the effect in all cases.

The color center model is proposed based on the observation of bleaching the absorption band of GODC and forming a new absorption band when the Ge-doped silica fiber is irradiated with a $248 \mathrm{~nm}$ laser [37]. The new absorption band is assigned to the formation of a color center, which is a photo-excited electron or hole trapped by a nearby defect center. The light-induced refractive index change is associated to the absorption change through the Kramers-Kronig relationship [38].

The densification model interprets the photosensitivity mechanism with UVinduced glass density change [39]. The bond breaking caused by laser irradiation alters the glass structural network, and leads to the densification as the result of the glass 
collapse. The increase in the glass density contributes to a positive refractive index change.

The Color center and densification models are two principal mechanisms for the photo-induced refractive index change. Other models were also found evident to be responsible for photosensitivity mechanism of optical fibers. Detailed information can be found in references $[26,33]$.

\subsection{Photosensitivity in Phosphate Glass Fibers}

\subsubsection{Laser-induced refractive index change in phosphate glasses and fibers}

The photosensitivity of phosphate glasses and fibers is of great interest because the phosphate glass is an ideal host medium for building compact high-gain amplifiers and lasers. The phosphate glasses have a high solubility of rare-earth ions without cluster formation. The cluster formation effect, which causes concentration quenching and degrades the gain, is detrimental to the laser efficiency and places a limit on the doping concentration of rare-earth ions in the host glasses. Compared to the maximum doping level of the silica glass $(<0.1 \mathrm{~mol} \%)$, phosphate glasses can incorporate doping concentrations of up to several wt. \% of Erbium and Ytterbium. However, the phosphate glasses were found to be lack of photosensitivity to conventional UV excimer lasers. As a result, few studies were engaged to investigate the photosensitivity of phosphate glasses and fibers. A summary is made here about these works attempting to induce refractive index change in phosphate glass or fiber samples with all possible irradiation laser sources. 
The photosensitivity of phosphate glass to the standard UV excimer laser irradiation was investigated by Pissadakis et al. [5]. Bragg gratings were written into both non ion-exchanged and ion-exchanged bulk glass samples by exposure with $248 \mathrm{~nm}$ nspulsed KrF laser through a phase mask. The phosphate glass exhibited poor photosensivity as a low refractive index modulation of only $\sim 10^{-5}$ was formed in the non ion-exchanged samples. Although the additional ion-exchange process aided to achieve a large refractive index modulation up to $2 \times 10^{-3}$, this change was confined in a shallow region with a depth of $\sim 3 \mu \mathrm{m}$. This physical size of the modified region is too small for forming good-quality waveguides or Bragg gratings.

Germanium co-doping, which is an effective method for photosensitization of silica glasses, demonstrates little success in phosphate glasses. Suzuki et al. have attempted to inscribe Bragg gratings into $30 \mathrm{wt} . \%$ Ge-doped phosphate glasses with 244 $\mathrm{nm} \mathrm{CW}$ light from a frequency-doubled $\mathrm{Ar}^{+}$ion laser [40]. Only weak gratings with refractive index changes of $3.5 \times 10^{-5}$ were obtained in glass samples with such high doping concentrations of germanium.

Our recent work demonstrated that strong Bragg gratings can be written in both $\mathrm{Er} / \mathrm{Yb}$ doped and undoped phosphate glasses and glass-based fibers by using an intense $193 \mathrm{~nm}$ ns-pulsed ArF excimer laser $[12,13,41]$. As for two conventional UV excimer lasers used for grating fabrication, $193 \mathrm{~nm}$ lasers can more readily induce refractive index change than $248 \mathrm{~nm}$ lasers because the light wavelength is closer to the deep UV absorption edge of the glasses. A refractive index modulation of $\sim 7 \times 10^{-5}$ was achieved for FBGs written in $\mathrm{Er} / \mathrm{Yb}$ undoped phosphate glass fibers. A promising advantage of these $193 \mathrm{~nm}$ irradiation-induced Bragg gratings in phosphate glass fibers is their thermal 
growth at high temperatures. The refractive index modulation of the grating was further increased to $\sim 1.4 \times 10^{-4}$ when subjected to a heating treatment at elevated temperatures of $\sim 170{ }^{\circ} \mathrm{C}$.

In addition to these conventional UV excimer lasers, high intensity ultrafast pulsed lasers have become prevalent in recent years for inducing the refractive index change in glass materials to fabricate waveguide or grating structures. The high pulse energy of femtosecond lasers facilitates nonlinear multi-photon absorption processes and produces large refractive index changes even in non-photosensitive materials. By using UV or IR fs-pulsed lasers and the phase mask technique, strong FBGs were successfully written in Er/Yb doped phosphate glass fibers in several studies [42-44]. Grobnic et al. achieved refractive index modulations of $1.5 \times 10^{-3}$ with a $800-\mathrm{nm}$ Ti:sapphire laser [43]. In a separate work, Hofmann et al. obtained FBGs with low level index modulations of $1.1 \times 10^{-4}$ with the 800 -nm Ti:sapphire laser [44]. The different photosensitivity response of these two works should be attributed to the difference in the laser irradiation condition and the chemical composition of the phosphate fibers.

One disadvantage of the aforementioned IR fs-laser induced FBGs is that only second- or third-order Bragg gratings can be formed for application at C-band wavelength. This arises from the fact that the wavelength of $800 \mathrm{~nm}$ light irradiation limits the spatial resolution of interference fringes used for imprinting gratings. With the use of a $248 \mathrm{~nm}$ fs-pulsed $\mathrm{KrF}$ laser, a standard first-order Bragg grating, the same as those fabricated by UV excimer lasers, was inscribed within the phosphate glass fibers by Sozzi et al. [42]. The laser-induced refractive index modulation of the grating was about $1.1 \times 10^{-4}$, while the average refractive index change was as large as $1 \times 10^{-3}$. 
In addition to fiber samples, Bragg gratings were also fabricated in bulk glass samples with the irradiation of fs-pulsed lasers [45]. In this work, Dekker et al. used point-by-point scanning method to inscribe first-order Bragg gratings with a $800-\mathrm{nm}$ Ti:sapphire laser, and an index modulation of $2.7 \times 10^{-4}$ was achieved in Yb-doped phosphate glasses.

More studies on modifying the refractive index of glasses with IR fs-pulsed lasers were focused on laser-written embedded waveguide structures in $\mathrm{Er} / \mathrm{Yb}$ doped phosphate bulk glass samples. Waveguides with large average refractive index changes $\left(>3 \times 10^{-3}\right)$ were successfully created in several different types of phosphate glasses [46-49]. However, differences in the refractive index change profiles exist among these studies. A modified region with negative index changes with similar amplitude was found along with that with positive index changes in [47], while only pure positive index changes were induced for waveguides in [48]. This suggests that the laser-material interaction between the fs-pulsed laser and the phosphate glass is a rather complicated process, which is highly dependent on the laser irradiation condition and glass composition.

All the above-mentioned studies on the photosensitivity of phosphate glasses and fibers are summarized in Table 2.1. For multicomponent glasses such as phosphate glasses, the difference in the chemical composition of the glasses used in different studies plays an important role in the photosensitivity of glasses. Compared to those FBGs written by fs-pulsed lasers, our $193 \mathrm{~nm}$ ArF excimer laser-induced FBGs have lower refractive index modulation $\left(\sim 1 \times 10^{-4}\right)$, but are still sufficient to produce practically useful reflection spectra. Another advantage of our standard UV-induced gratings is the excellent quality of spectral shapes of grating transmission and reflection. In contrast, the 
inscription of high intensity fs-laser induced gratings involves glass damage and makes them Type II gratings, which have poor quality grating spectra and have additional scattering loss.

\begin{tabular}{|c|c|c|c|c|}
\hline Laser Source & $\begin{array}{l}\text { CW or } \\
\text { Pulsed }\end{array}$ & $\begin{array}{l}\text { Glass composition } \\
\text { and sample }\end{array}$ & $\begin{array}{c}\text { Refractive index } \\
\text { change }\end{array}$ & Refs \\
\hline 193 nm, ArF laser & ns-pulsed & $\begin{array}{l}\mathrm{Er} / \mathrm{Yb} \text { doped and } \\
\text { undoped phosphate } \\
\text { glasses and fibers }\end{array}$ & $\begin{array}{l}\text { Bragg grating } \\
\Delta n_{\text {mod }} \sim 1 \times 10^{-4}\end{array}$ & $\begin{array}{c}\text { Our } \\
\text { work }[12,13,41]\end{array}$ \\
\hline $248 \mathrm{~nm}, \mathrm{KrF}$ laser & ns-pulsed & $\begin{array}{l}\text { Er doped } \\
\text { ion-exchanged } \\
\text { phosphate glass }\end{array}$ & $\begin{array}{c}\text { Bragg grating } \\
\Delta n_{\text {mod }} \sim 10^{-5} \text { (non } \\
\text { ion-exchanged) } \\
\Delta n_{\text {mod }}=2 \times 10^{-3} \\
\text { (ion-exchanged) }\end{array}$ & {$[5]$} \\
\hline $\begin{array}{c}244 \mathrm{~nm}, \\
\text { frequency-doubled } \\
\mathrm{Ar}^{+} \text {ion laser }\end{array}$ & $\mathrm{CW}$ & $\begin{array}{c}30 \text { wt. } \% \mathrm{GeO}_{2} \\
\text { phosphate glass }\end{array}$ & $\begin{array}{c}\text { Bragg grating } \\
\Delta n_{\text {mod }}=3.5 \times 10^{-5}\end{array}$ & [40] \\
\hline $248 \mathrm{~nm}, \mathrm{KrF}$ laser & fs-pulsed & $\begin{array}{c}\mathrm{Er} / \mathrm{Yb} \text { doped } \\
\text { phosphate fiber }\end{array}$ & $\begin{array}{c}\text { Bragg grating } \\
\Delta n_{\text {mod }}=1.1 \times 10^{-4} \\
\Delta n_{\text {avg }}=1 \times 10^{-3}\end{array}$ & [42] \\
\hline $\begin{array}{c}800 \mathrm{~nm}, \\
\text { Ti:sapphire laser }\end{array}$ & fs-pulsed & $\begin{array}{c}\mathrm{Er} / \mathrm{Yb} \text { doped } \\
\text { phosphate fiber }\end{array}$ & $\begin{array}{c}\text { Bragg grating } \\
\Delta n_{\text {mod }}=1.5 \times 10^{-3}\end{array}$ & {$[43]$} \\
\hline $\begin{array}{c}800 \mathrm{~nm}, \\
\text { Ti:sapphire laser }\end{array}$ & fs-pulsed & $\begin{array}{c}\mathrm{Er} / \mathrm{Yb} \text { doped } \\
\text { phosphate fiber }\end{array}$ & $\begin{array}{c}\text { Bragg grating } \\
\Delta n_{\text {mod }}=1.1 \times 10^{-4}\end{array}$ & {$[44]$} \\
\hline $\begin{array}{c}800 \mathrm{~nm}, \\
\text { Ti:sapphire laser }\end{array}$ & fs-pulsed & $\begin{array}{c}\text { Yb doped } \\
\text { phosphate glass }\end{array}$ & $\begin{array}{c}\text { Bragg grating } \\
\Delta n_{\text {mod }}=2.7 \times 10^{-4}\end{array}$ & {$[45]$} \\
\hline $\begin{array}{c}800 \mathrm{~nm}, \\
\text { Ti:sapphire laser }\end{array}$ & fs-pulsed & $\begin{array}{l}\text { Kigre Yb:QX, } \\
\text { Schott IOG-1, zinc } \\
\text { phosphate glass }\end{array}$ & $\begin{array}{c}\text { Waveguide } \\
\Delta n_{a v g}>3 \times 10^{-3} \\
\text { and } \Delta n_{\text {avg }}<- \\
\quad 3 \times 10^{-3}\end{array}$ & [46-49] \\
\hline
\end{tabular}

Table 2.1 Summary of photosensitivity in phosphate glasses and fibers under different irradiation laser sources.

\subsubsection{Phosphate glasses structure}

As discussed in the previous subsection, the photosensitivity of phosphate glasses is highly dependent on the chemical composition of the glasses. A brief introduction to 
phosphate glass composition and network structure made here is necessary for understanding the photosensitivity mechanisms of the glasses.

Phosphate glasses are mainly based on phosphorus pentoxide $\left(\mathrm{P}_{2} \mathrm{O}_{5}\right)$, usually with other chemical compounds. The phosphate fibers used in this work were drawn at the University of Arizona. The core and cladding preforms were based on the phosphate glass similar to that reported in reference [50], which has a base glass composition of 62 $\mathrm{mol} \% \mathrm{P}_{2} \mathrm{O}_{5}, 12 \mathrm{~mol} \% \mathrm{Al}_{2} \mathrm{O}_{3}$, and $26 \mathrm{~mol} \% \mathrm{Li}_{2} \mathrm{O}+\mathrm{Na}_{2} \mathrm{O}+\mathrm{BaO}+\mathrm{CaO}$. These oxides are generally classified into three categories for their roles in glass network formation: $\mathrm{P}_{2} \mathrm{O}_{5}$ as network formers, which form the highly cross-linked network through covalent P-O bonds; $\mathrm{Al}_{2} \mathrm{O}_{3}$ as network intermediates, which can act as network formers or modifiers because aluminum can form either covalent or ionic bonds with oxygen; $\mathrm{Li}_{2} \mathrm{O}+\mathrm{Na}_{2} \mathrm{O}+$ $\mathrm{BaO}+\mathrm{CaO}$ (oxides of alkali and alkaline earth metals) as network modifiers, which terminate the connections of network by ionic bonds between metals and oxygen atoms.

The basic structure of a phosphate glass matrix consists of a short-range continuous random network of phosphorus tetrahedral units. Each phosphorus tetrahedron, with one phosphorus atom surrounded by four oxygen atoms, is linked together with neighboring phosphorus tetrahedra through corner-shared oxygen atoms. Because of the pentavalence of phosphorus, the phosphorus atom has to form a double bond $(\mathrm{P}=\mathrm{O})$ with one oxygen atom, which is called non-bridging oxygen $(\mathrm{NBO})$ as it terminates the network cross-link. The remaining three oxygen atoms can link to neighboring phosphorus atoms through forming a P-O-P bond, and hence are called bridging oxygen (BO). The introduction of glass modifiers such as metal ions can break 
the P-O bond and form an ionic bond between metal and oxygen atoms, and convert oxygen atoms from $\mathrm{BO}$ to $\mathrm{NBO}$.

Phosphorus tetrahedral units can be classified using $\mathrm{Q}^{\mathrm{i}}$ terminology, where $\mathrm{i}$ represents the number of bridging oxygen atoms [47,48,51]. All 4 possible forms of phosphorus tetrahedron including $\mathrm{Q}^{3}$ (cross-linked), $\mathrm{Q}^{2}$ (intermediate), $\mathrm{Q}^{1}$ (terminal), $\mathrm{Q}^{0}$ (isolated) units are illustrated in Figure 2.6. The $\mathrm{O}$ atoms with one electron in $\mathrm{Q}^{\mathrm{i}}$ units are non-bridging oxygen atoms which form ionic bonds with nearby metal ions. The proportion of each type of $\mathrm{Q}^{\mathrm{i}}$ units is determined by the glass composition, specifically the ratio of oxygen atoms to phosphorus atoms $([\mathrm{O} / \mathrm{P}]$ ratio) $[51]$. With variation of the $[\mathrm{O} / \mathrm{P}]$ ratio from 2.5 to 4 , the main component forming the glass network varies from a cross-linked $\mathrm{Q}^{3}$ tetrahedral unit to an isolated $\mathrm{Q}^{0}$ tetrahedral unit. Correspondingly, the glass network is depolymerized to short chains as more modifiers are introduced.
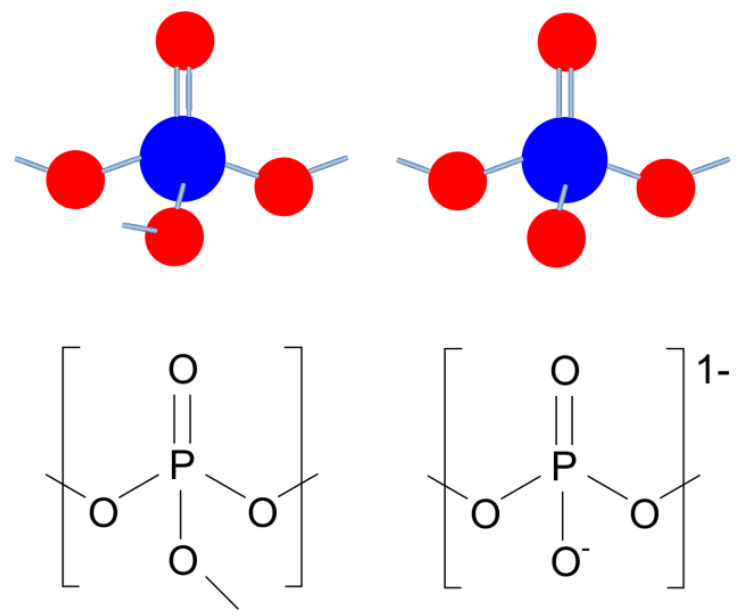

$Q^{3}$
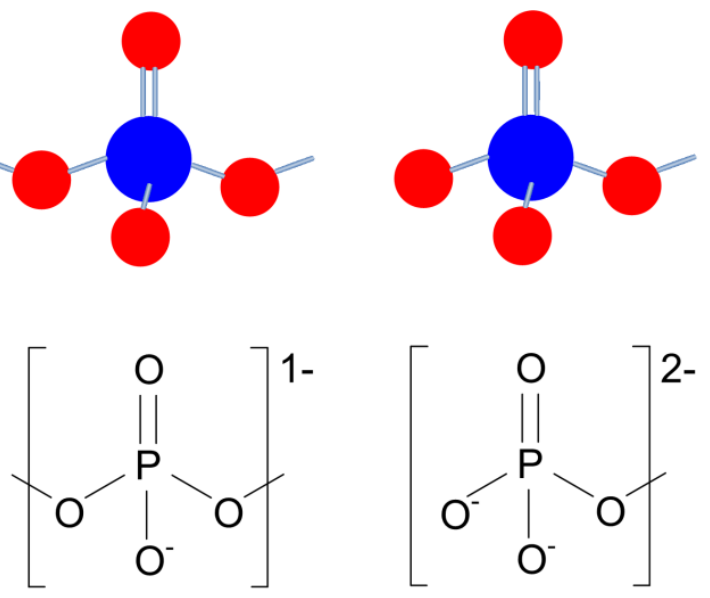

$Q^{2}$

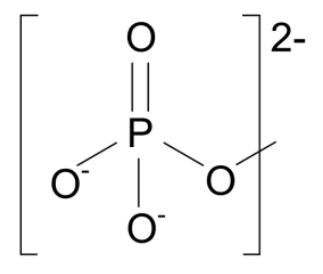

$Q^{1}$
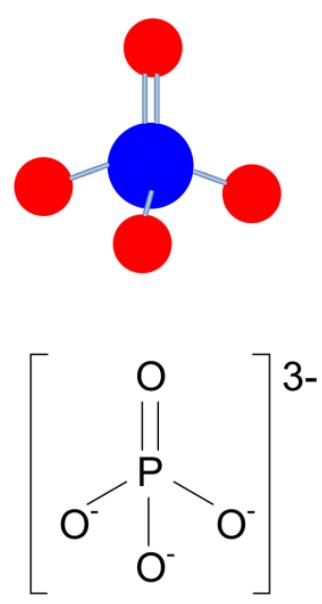

$Q^{0}$

Figure 2.6 Phosphorus tetrahedral units in phosphate glass network.

For the phosphate glass fiber investigated in this work, of which $[\mathrm{O}] /[\mathrm{P}]=3$ or slightly $<3$, the network of this phosphate glass is mainly formed by $\mathrm{Q}^{2}$ units of 
phosphorus [50]. In this type of glass, $\mathrm{Q}^{2}$ units are linked together to form long polymerlike chains terminated by $\mathrm{Q}^{1}$ units. Compared to silica glass whose network is highly cross-linked via all $4 \mathrm{O}$ atoms in a tetrahedral unit, the phosphate glass has a loose and open network so it can accommodate higher concentrations of rare-earth elements. On the other hand, this feature of the network makes the phosphate glasses soft glasses, which exhibit low physical strength, and low transient temperature $\left(\sim 448{ }^{\circ} \mathrm{C}\right)$ and softening temperature $\left(\sim 475^{\circ} \mathrm{C}\right)[50]$.

\subsubsection{Mechanisms of photosensitivity in phosphate glasses}

Similar to the case of silica glasses, the exact mechanisms of photosensitivity in phosphate glasses is still not well understood. The origin of photo-induced refractive index changes in phosphate glasses has been investigated with different diagnostic tools. Different models have been proposed to explain the photosensitivity of phosphate glasses. Most studies suggest that the photosensitivity of phosphate glasses is a rather complicated process which usually involves the interaction of multiple mechanisms [47,49]. The Color center model and the densification model are commonly accepted for explaining photo-induced refractive index changes in phosphate glasses.

\subsubsection{Color center model}

The formation of color centers is widely observed in phosphate glasses upon UV irradiation or high intensity femtosecond-laser irradiation [47,48,52-55]. This model proposes that the light irradiation causes the photoionization of the phosphate glass material through single- or multiple-photon absorption depending on irradiation conditions and glass composition. This photoionization process generates free electrons and holes, which can travel in the glass network and can be trapped at defect precursors 
to form the color centers. These color centers, including electron centers and hole centers, cause the strong absorption in the UV and the visible regions. The photo-induced refractive index change of glasses can be explained and associated with these absorption bands formed at short-wavelength regions through the Kramers-Kronig relationship.

The color centers formed in phosphate glasses include the phosphorus-oxygen hole center $(\mathrm{POHC})$ and three types of electron centers such as $\mathrm{PO}_{2}, \mathrm{PO}_{3}$ and $\mathrm{PO}_{4}$ color centers. The POHC has broad absorption bands at visible regions, which has characteristic wavelengths of $330 \mathrm{~nm}, 430 \mathrm{~nm}$ and $540 \mathrm{~nm}$. Other color centers $\mathrm{PO}_{2}, \mathrm{PO}_{3}$ and $\mathrm{PO}_{4}$ have absorption bands at $270 \mathrm{~nm}, 210 \mathrm{~nm}$ and $240 \mathrm{~nm}$ respectively [54,56]. Figure 2.7 shows one example of UV irradiation-induced absorption spectra of different color centers in phosphate glasses. Although the irradiation source used for this measurement was a UV lamp [54], the observation of the same color center species was also found using other irradiation sources such as UV excimer lasers and fslasers $[47,48,52-55]$. As seen in the figure, the absorption bands of different color centers may overlap with each other and are then difficult to distinguish. The identification and characterization of color center species is usually performed with supplemental diagnostics tools. For paramagnetic color centers such as these P-related color centers, which are not neutrally charged because of additional trapped electron or hole, electron spin resonance (ESR) can be used to detect the non-zero electron spin angular momentum of the unpaired electron [56]. 


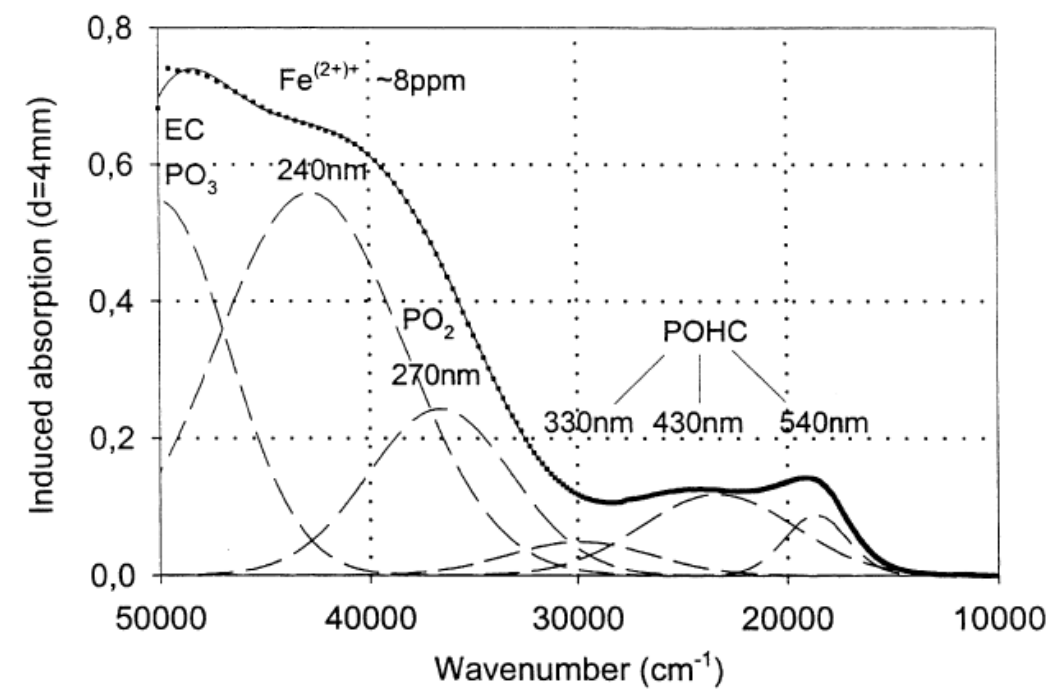

Figure 2.7 UV lamp irradiation-induced color centers in phosphate glass. The irradiationinduced absorption spectra (line) is decomposed into Gaussian peaks (dashed lines) of different color centers [54].

Among all these P-related color center species, POHC is more commonly observed after light irradiation $[47,48,55]$. It is formed by a free hole trapped at the nonbridging oxygen of the $\mathrm{Q}^{2}$ phosphorus tetrahedron. Detailed information relating to the chemical structure of all P-related color center species can be found in references $[54,56]$. As explained in the previous subsection, this $\mathrm{Q}^{2}$ phosphorus tetrahedron is the major structural network unit in the metaphosphate glass studied in this work. It is expected that the formation of the POHC will be also present in the case of our fiber samples, which is verified by our work in Chapter 4 .

\subsubsection{Densification model}

In addition to the color center formation, many studies found that the photoinduced refractive index change of phosphate glasses is also attributed to density change resulting from glass network modification $[41,42,47,57,58]$. The laser irradiation can cause bond breaking of phosphate glass network. As described in the subsection 2.4.2, the phosphate glass network consists of long polymer-like chains of linked $\mathrm{Q}^{2}$ tetrahedra. 
The change observed in the Raman spectra of glasses upon irradiation indicates that the P-O-P bond linkage between the tetrahedra has been broken under laser irradiation [41]. As a result, the long polymer-like chain structure of glass network is shorten and collapses, and glass densification is formed. As a result, a positive refractive index change was observed in phosphate glass after $193 \mathrm{~nm}$ UV irradiation.

However, as multicomponent glasses, the photosensitivity of phosphate glasses is a process more complicated than that of silica glasses. Some other studies suggest that the network modification caused by laser irradiation leads to an opposite behavior in terms of glass density [42,47]. By measuring the Knoop hardness or the Raman spectra of irradiated glass samples, these studies also observed the network modification of phosphate glasses. But, an expansion of the modified glass network was proposed in order to explain the negative refractive index change observed in the irradiation region. This different behavior in the compaction or expansion of glasses is the result of differences in the glass composition and the detailed laser irradiation conditions.

In summary, the photosensitivity of phosphate glasses is usually explained by the color center model and densification model. Most studies found that the photo-induced refractive index change is the result of contributions from both models. 


\section{Chapter 3}

\section{Photosensitivity and Thermal Stability of FBGs in}

\section{Phosphate Glass Fibers}

This chapter presents a systematic investigation of photosensitivity and thermal stability of UV-induced FBGs in phosphate glass fibers. Thermal growth of reflectivity of seed gratings in phosphate fibers is observed upon annealing at temperatures up to $250{ }^{\circ} \mathrm{C}$. A recipe for reliably obtaining strong gratings with index modulations of $\sim 1 \times 10^{-4}$ is proposed. The major work in this chapter has been previously reported in two papers $[59,60]$.

\subsection{Introduction}

Rare-earth-doped phosphate glass (without silica) is an important medium for high power amplifiers and compact fiber lasers because of the higher solubility of rare-earth elements relative to silica glass [2]. However, the lack of conventional photosensitivity in phosphate glass fibers hindered the integration of UV-written intra-fiber gratings into the laser cavity. One alternative way to inscribe gratings in phosphate glass is to resort to high energy femtosecond lasers. With the use of femtosecond lasers at wavelengths of $248 \mathrm{~nm}$ or $800 \mathrm{~nm}$, laser-inscribed waveguide structures in bulk glasses and even strong Bragg gratings in phosphate fibers have been fabricated [42-44,47]. However, it is still of fundamental interest to investigate the photosensitivity of phosphate fibers to UV excimer lasers. In recent previous work, it was demonstrated that the direct writing of 
gratings in both undoped and $\mathrm{Er} / \mathrm{Yb}$ doped (hereafter referred to as "passive" and "active") phosphate glass fibers was possible using an ArF excimer laser [12,13], and further, it was shown that the grating index modulation in phosphate glass fibers can be strengthened by heating the gratings to temperatures between 100 and $250{ }^{\circ} \mathrm{C}$ [60]. This kind of photo-thermal process allows the formation of gratings with reflectivity above $90 \%$ and the fabrication of compact distributed feedback (DFB) lasers in active phosphate glass fibers $[24,25]$.

In this chapter, we systematically investigate the grating fabrication to determine under which conditions thermal growth is achievable by thermal annealing of seed Bragg gratings in passive and active phosphate glass fibers. In particular, we show that the UV irradiation time is the critical parameter in achieving strong photo-thermal growth, regardless of the index modulation amplitude or contrast of the seed grating, and regardless of the core dopants. It is also shown that for the first part of the process (the UV induced index change), the photosensitivity of the passive fibers is about two times higher than that of the active fibers.

\subsection{Fiber Bragg Grating Fabrication Setup}

The experiment setup for fabricating fiber Bragg gratings with phase mask technology is schematically illustrated in Figure 3.1. The fiber Bragg grating writing system consists of the laser source, the beam-shaping optics, the phase mask and the fiber mounting stage. 


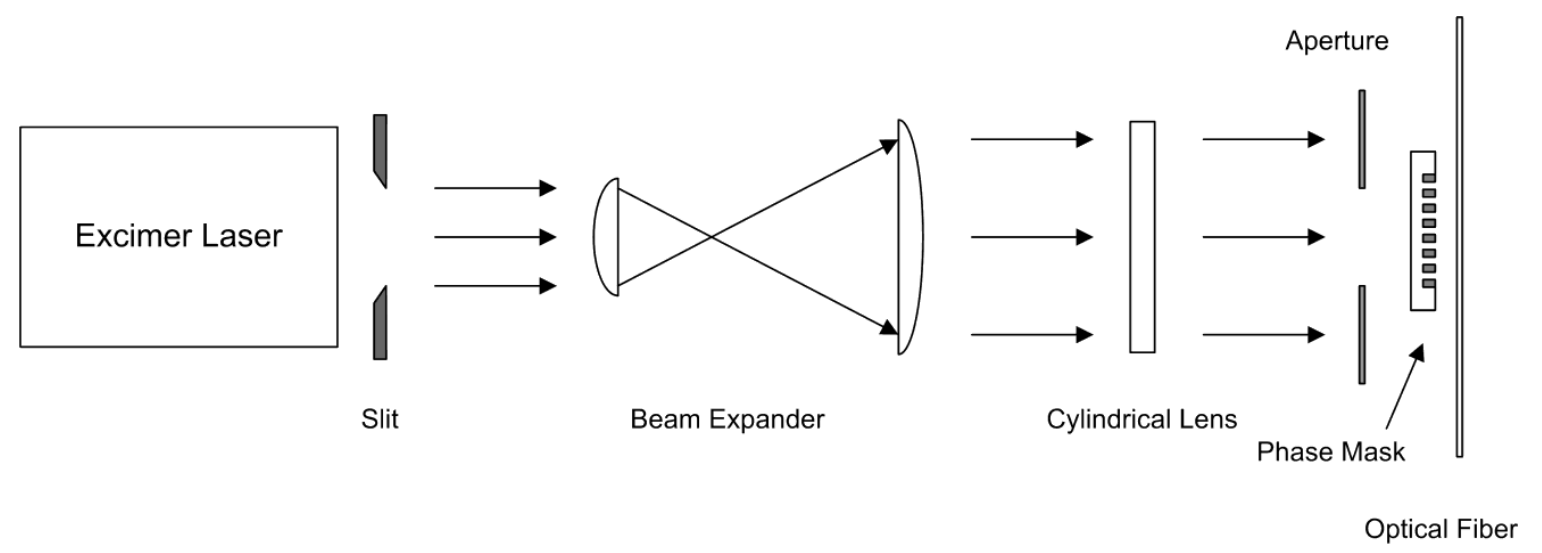

Figure 3.1 Schematic of $193 \mathrm{~nm}$ excimer laser fiber Bragg grating fabrication system.

A switchable excimer laser (PulseMaster 840 series from GSI Lumonics), which can operate at the wavelength $248 \mathrm{~nm}(\mathrm{KrF})$ or $193 \mathrm{~nm}(\mathrm{ArF})$, provides intense UV light for the inscription of the fiber Bragg gratings. For grating fabrication in phosphate glass fibers, a $193 \mathrm{~nm}$ wavelength model is used. The pulse energy can achieve up to $100 \mathrm{~mJ}$ with pulse duration of around $20 \mathrm{~ns}$, and the repetition rate can be varied up to $100 \mathrm{~Hz}$.

A slit is placed in front of the laser outlet in order to select the central uniform region of the laser beam. The slit dimensions are precisely adjusted to $15.55 \mathrm{~mm}$ by 1.07 $\mathrm{mm}$, which is only a small portion of the laser beam size. The slit blocks a large surrounding part of the UV light, which can not be used to illuminate the fiber even with the presence of a focus lens. This arrangement also makes the alignment of the following optics elements easier.

The laser beam is then enlarged by a beam expander, which consists of two cylindrical lenses with focal lengths of $50 \mathrm{~mm}$ and $150 \mathrm{~mm}$ respectively. Passing through this component, the beam size will be magnified 3 times in the horizontal direction and unchanged in the vertical direction. This component makes the beam intensity 
distribution more uniform, which is necessary for fabricating better quality uniform FBGs. It also acts as beam shaper to adjust the output of the laser, which is slightly divergent, to a beam of parallel light.

After the beam expander, another perpendicular cylindrical lens is used to focus the interference pattern along the fiber. Its focus length is $100 \mathrm{~mm}$. The incorporation of this focus lens is intended to make more efficient use of the laser beam, and typically in our setup the pulse fluence illuminating the fiber is improved about 20 times. The focused beam at fiber position becomes a very thin narrow beam with size of $46.65 \mathrm{~mm}$ by $50.4 \mu \mathrm{m}$, and the fluence per pulse is approximately $45 \mathrm{~mJ} / \mathrm{cm}^{2}$.

As a key part of fabrication system, the phase mask is mounted on a motion stage. The alignment of the phase mask is extremely important for inscribing a high quality fiber Bragg grating. All three rotational directions of the phase mask are provided by the motion stage in order to allow precise alignment of the phase mask perpendicular to the incident beam propagating axis. The period of the phase mask used to write the FBGs is $988.4 \mathrm{~nm}$. With the zeroth-order diffracted beam suppressed by optimizing the design of phase mask, the plus and the minus first-order diffracted beams will form an interference fringe pattern with a period of $494.2 \mathrm{~nm}$. The interference fringes will photo-imprint a periodic pattern in the phosphate fiber and a FBG with a grating period of $494.2 \mathrm{~nm}$ will be formed. An aperture is placed right in front of the phase mask in order to adjust the width of the UV laser beam and hence the grating length of the formed FBG.

Phosphate fibers are prepared as 5-cm-long samples, and are spliced to standard telecommunication (CORNING SMF28) fiber pigtails. The fiber chain is clamped between the two fiber holders of the fiber mounting stage. While the fiber is mounted, a 
tension of $20 \mathrm{~g}$ is applied to the fiber in order to keep it straight and therefore form a precise fringe pattern on the fiber. With the aid of the mounting stage, the fiber is aligned in all directions such that it is placed along the focused interference fringe. Moreover, the optical fiber is placed in close proximity to the phase mask, with the distance between the two typically around $100 \mu \mathrm{m}$. The parameters of the grating fabrication setup are summarized in Table 3.1.

\begin{tabular}{|c|c|}
\hline Laser Source & $193 \mathrm{~nm} \mathrm{ArF}$ \\
\hline Beam Size* & $46.65 \mathrm{~mm} \times 50.4 \mu \mathrm{m}$ \\
\hline Pulse Duration (ns) & $\sim 20$ \\
\hline Pulse Fluence (mJ/cm $\left.{ }^{\mathbf{2}}\right)$ & $\sim 45$ \\
\hline Repetition Rate (Hz) & 50 or 100 \\
\hline Phase Mask Period (nm) & 988.4 or 982.0 \\
\hline
\end{tabular}

* Beam size focused at fiber position; the beam length can be adjusted by an aperture.

Table 3.1 Parameters for grating inscription in phosphate glass fibers.

In the course of inscribing fiber Bragg gratings, the grating transmission spectra are monitored and recorded continually by launching light from a Broadband Source (from JDSU) in the fiber and measuring the transmitted light with an optical spectrum analyzer (OSA, Yokogawa AQ 6317B). A LabVIEW program has been compiled on a computer to control the OSA and record the measured data. The experimental setup of insitu characterization of FBGs transmission spectra during grating inscription is shown in Figure 3.2.

The growth evolution of the average refractive index change and the refractive index modulation of the FBG can be calculated from the measured position and 
amplitude of the reflection resonance in the transmission spectra. For a uniform FBG, the average refractive index change $\Delta \mathrm{n}_{\text {avg }}$ and refractive index modulation $\Delta \mathrm{n}_{\text {mod }}$ are determined by Eq. (2.11) and (2.13). A confinement factor $\Gamma$ of 0.75 for phosphate fiber core mode is used in the calculation.

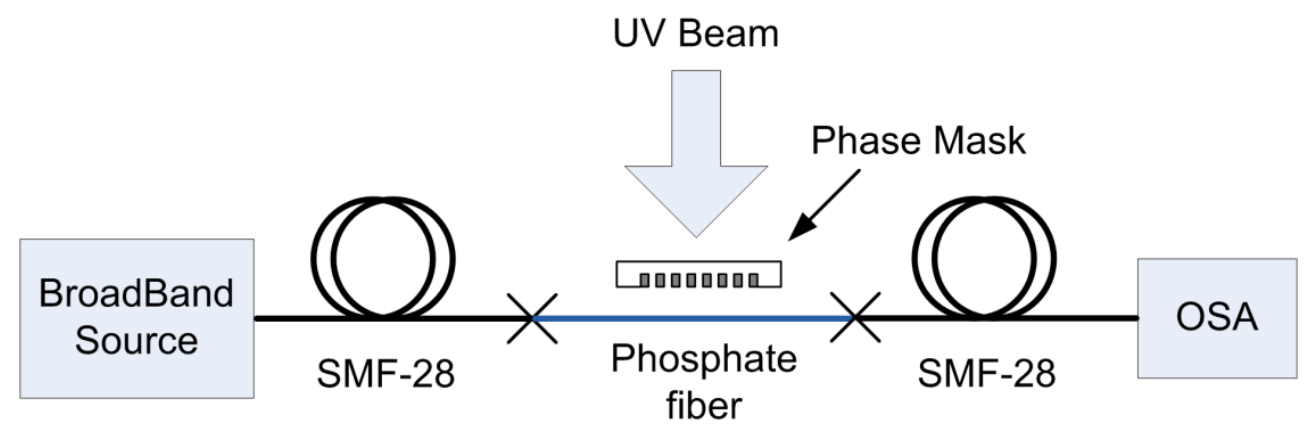

Figure 3.2 Experimental setup for in-situ measurement of transmission spectra of FBGs in phosphate fibers during the inscription process.

\subsection{Phosphate Glass Fiber Type under Test}

Grating fabrication experiments were performed on two types of fiber samples: phosphate fiber with Er/Yb doped core (Fiber A, A for active) and undoped phosphate fiber (Fiber P, $\mathrm{P}$ for passive). The $\mathrm{Er} / \mathrm{Yb}$ doped fiber has a highly doped core with $1 \mathrm{wt} \%$ $\mathrm{Er}_{2} \mathrm{O}_{3}$ and $8 \mathrm{wt} \% \mathrm{Yb}_{2} \mathrm{O}_{3}$ (corresponding to doping concentrations of $1.1 \times 10^{26}$ and $8.6 \times 10^{26}$ ions $/ \mathrm{m}^{3}$ respectively), while the undoped fiber is free of $\mathrm{Er} / \mathrm{Yb}$ dopants in the core. The details of the fibers are summarized in Table 3.2. These fibers are designed as single mode fibers at $1550 \mathrm{~nm}$ band, whereas there is still a modal field diameter mismatch between phosphate fibers and standard telecommunication (CORNING SMF28) fiber. 


\begin{tabular}{|c|c|c|c|c|c|}
\hline \multirow{2}{*}{$\begin{array}{c}\text { Fiber } \\
\text { Type }\end{array}$} & $\begin{array}{c}|c| \\
\mathrm{Er}^{3+} \\
\left(\mathrm{ions} / \mathrm{m}^{3}\right)\end{array}$ & $\begin{array}{c}\text { Core Dopants } \\
\left(\mathrm{ions} / \mathrm{m}^{3}\right)\end{array}$ & $\begin{array}{c}\text { Core } \\
\text { Diameter } \\
(\mu \mathrm{m})\end{array}$ & $\begin{array}{c}\text { Cladding } \\
\text { Diameter } \\
(\mu \mathrm{m})\end{array}$ & $\begin{array}{c}\mathrm{n}_{\text {eff }} \text { at } \\
1550 \mathrm{~nm}\end{array}$ \\
\hline $\mathrm{A}$ & $1.1 \times 10^{26}$ & $8.6 \times 10^{26}$ & 11 & 125 & 1.562 \\
\hline $\mathrm{P}$ & None & None & 7.6 & 125 & 1.566 \\
\hline
\end{tabular}

Table 3.2 Details of phosphate fibers used for grating inscription.

Phosphate fibers were prepared as 5-cm-long samples, and were spliced to standard telecommunication (CORNING SMF28) fiber pigtails. Bragg gratings were written in both types of phosphate glass fibers using the grating fabrication setup as described in Section 3.2. A phase mask with a period of $988.4 \mathrm{~nm}$ was used to inscribe gratings with length of $2 \mathrm{~cm}$. The ArF excimer laser was set to operate at a repetition rate of $50 \mathrm{~Hz}$. During the grating inscription, the grating transmission spectrum was recorded continually, and the growth evolution of the average refractive index change $\Delta \mathrm{n}_{\text {avg }}$ and refractive index modulation $\Delta \mathrm{n}_{\text {mod }}$ the FBG would be calculated accordingly.

\subsection{Fiber Bragg Grating Fabrication in Phosphate Fibers}

\subsubsection{FBGs written in phosphate fibers}

Figure 3.3 illustrates the transmission spectra of 2-cm-long UV-induced FBGs in both passive and active phosphate glass fibers. The transmission spectra are normalized to the transmission of pristine fiber samples before UV irradiation. As can be seen in the figure, an excess loss of fiber samples is induced by UV irradiation, in addition to the formation of FBGs. This point will be addressed in the following subsection. 


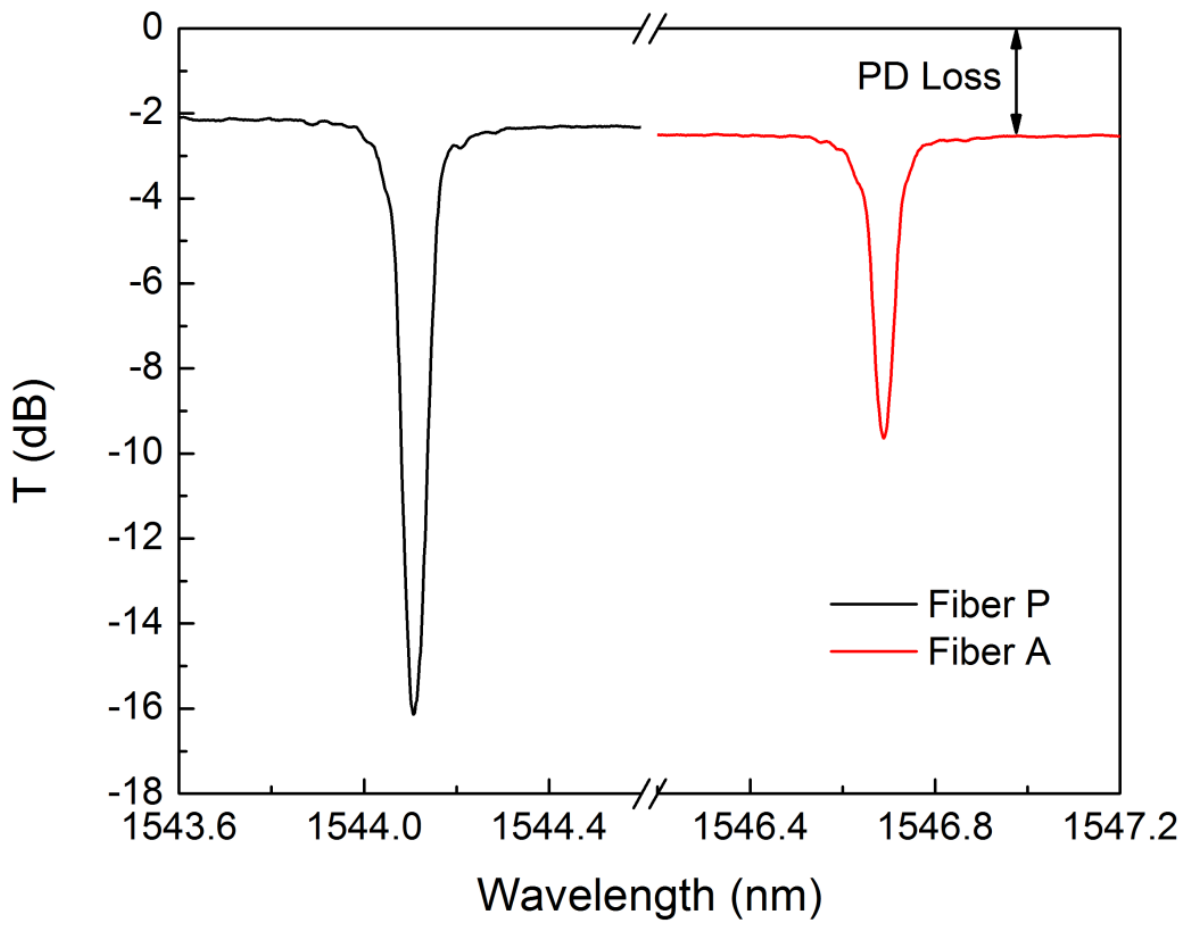

Figure 3.3 Transmission spectra of 2-cm-long UV-induced FBGs in passive and active phosphate fibers.

For the passive phosphate fiber, a grating with transmission notch depth of 14.3 $\mathrm{dB}$ (corresponding to reflectivity of $96.3 \%$ ) is inscribed by UV irradiation for 1083 seconds. The $3 \mathrm{~dB}$ bandwidth of the FBG is about $0.086 \mathrm{~nm}$. In contrast, the active phosphate fiber exhibits a lower photosensitivity than the passive fiber. After UV irradiation for 900 seconds, the grating written in Fiber A has a transmission notch depth of $7.2 \mathrm{~dB}$ (corresponding to reflectivity of $80.9 \%$ ), and a $3 \mathrm{~dB}$ bandwidth of $0.054 \mathrm{~nm}$. The Bragg wavelengths are $1544.108 \mathrm{~nm}$ and $1546.690 \mathrm{~nm}$ for gratings in Fiber P and A respectively. The difference in Bragg wavelength is the result of the different effective refractive indices $n_{\text {eff }}$ of two types of fibers as show in Table 3.2. A more detailed investigation on UV photosensitivity of both types of phosphate glass fibers will be presented in the next Section. 


\subsubsection{Side effect of FBG inscription: Photodarkening loss}

As shown in Figure 3.3, a remarkable UV-induced excess loss is observed during the grating inscription with the $193 \mathrm{~nm}$ laser irradiation. For both passive and active fiber samples, the excess loss shows a similar level of about $2 \mathrm{~dB}$, which corresponds to an attenuation coefficient of $1 \mathrm{~dB} / \mathrm{cm}$ by considering the irradiation beam length of $2 \mathrm{~cm}$. Furthermore, this UV-induced excess loss is not limited in wavelength ranges close to Bragg wavelength. A measurement of grating transmission spectra over the C-band demonstrates that it is a flat and broadband excess loss across the whole C-band (1525 $1565 \mathrm{~nm})$.

This UV-induced excess loss is a novel observation for grating inscription with UV excimer laser irradiation. Typically, the gratings fabricated in the silica glass fibers by UV irradiation are free of UV-induced absorption losses at the C-band. It is therefore a unique feature of phosphate glass fibers. However, this side effect of UV irradiation for FBGs inscription in phosphate fibers is undesired, because it introduces an additional internal cavity loss for a cavity built with UV-induced FBGs. This excess loss will be further investigated in Chapter 4. The origin of this loss is identified as photodarkening (PD) effect in phosphate fibers, and more details will be presented in that chapter.

\subsubsection{Side effect of FBG inscription: Laser ablation}

Another side effect of grating inscription in phosphate fibers is the laser ablation. Examination of the irradiated phosphate FBG samples with an optical microscope reveals that a thin and long surface damage is caused by $193 \mathrm{~nm}$ laser irradiation. Figure 3.4 shows microscope images of UV-induced damage on the phosphate fiber cladding surface for FBG samples in active and passive fibers. Figure 3.4(a) illustrates the 
microscope image around one end of FBG1 in Fiber A. A clear difference on fiber surface can be seen between the non-irradiated region (on the left) and the irradiated region (on the right). The spatial distribution of damage along the fiber axis is not uniform as shown in Figure 3.4(b), and is also different from that in Figure 3.4(a). This
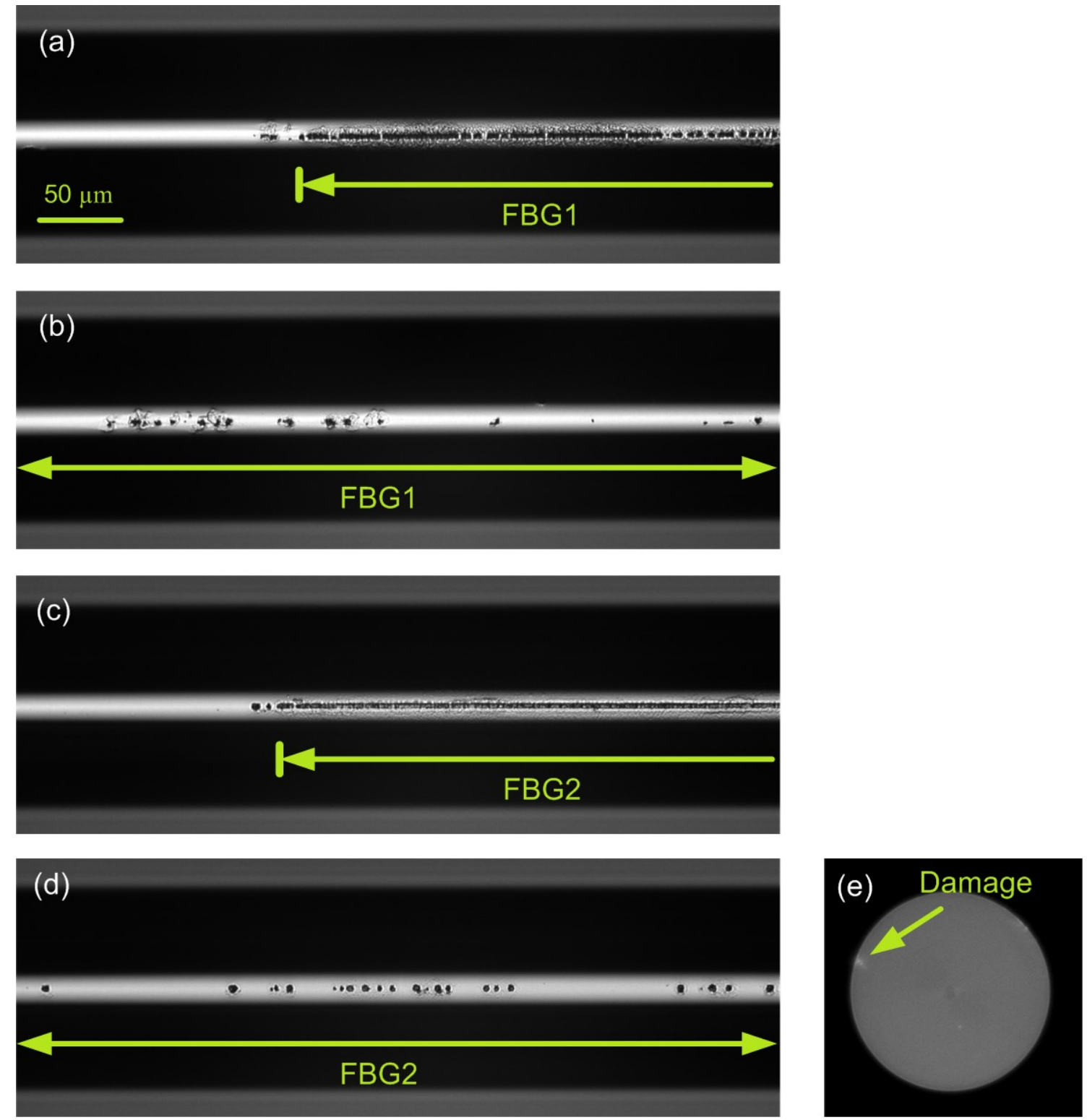

Figure 3.4 Microscope images of UV-induced damages on the phosphate fiber cladding surface for (a) one end and (b) a middle section of a sample FBG1 in Fiber A; (c) one end and (d) a middle section of a sample FBG2 in Fiber P; (e) cross-section of the cleaved FBG2. 
difference can be the result of several factors such as nonuniform intensity distribution of UV irradiation and imperfect homogeneity of these laboratory-made phosphate fiber samples. Similar behavior is also observed in Fiber P as shown in Figure 3.4(c) and (d). The UV-induced damage has a thickness of $\sim 4.5 \mu \mathrm{m}$, but it is just a shallow surface damage with depth of $4.6 \mu \mathrm{m}$ as seen in Figure 3.4(d). Consequently, this side effect is of little significance in affecting the grating fabrication in phosphate fibers.

This UV-induced damage is attributed to the laser ablation, which results in the removal of material from the surface by vaporization. The laser ablation was barely observed in UV-induced FBGs in silica fibers. For a fused silica fiber preform, the threshold pulse fluence of ablation with ArF excimer laser was found to be at the order of $\mathrm{J} / \mathrm{cm}^{2}$ [61]. In contrast, the phosphate glass, as a soft glass, requires lower threshold pulse fluence for laser ablation [62]. As a result, laser ablation readily occurs here for the pulse fluence $\left(\sim 47 \mathrm{~mJ} / \mathrm{cm}^{2}\right)$ used for grating fabrication.

\subsection{Photosensitivity and Thermal Stability of FBGs in Phosphate Fibers}

Figure 3.5 illustrates the growth of the average refractive index change $\Delta \mathrm{n}_{\text {avg }}$ and refractive index modulation $\Delta \mathrm{n}_{\text {mod }}$ during grating fabrication on both passive and active phosphate fibers. For the sake of easy comparison, the $\Delta \mathrm{n}_{\text {avg }}$ of passive and active fiber samples is plotted side by side with the same scale. The $\Delta \mathrm{n}_{\text {mod }}$ for both fibers is also presented with an enlarged scale compared to $\Delta \mathrm{n}_{\text {avg }}$, in order to show the details of growth pattern. Gratings P1-P4 and A1-A4 were fabricated with the same grating fabrication setup, aligned to generate strong grating index modulations. Gratings P5-P8 
were fabricated with an earlier setup that led to premature saturation of the fringe contrast and hence of the $\Delta \mathrm{n}_{\bmod }$ as reported earlier [60].

Except for $\Delta \mathrm{n}_{\text {mod }}$ of samples P5 and P6, the growth curves of all the samples in each graph follow the same stretched exponential function (calculated from samples P1P3 and A1-A3 only), within the $+/-95 \%$ prediction band limits. It is believed that remaining small differences in the grating growth patterns within each fiber type can be attributed to irradiation conditions from the excimer laser, such as pulse energy variations, UV beam spatial stability, and imperfect homogeneity of these laboratorymade phosphate fiber samples.

The refractive index changes follow nearly monotonic (again, apart from P5-P6) growth curves without showing signs of saturation for long UV irradiation times (up to $\sim 1100 \mathrm{~s}$ ). Small oscillations in almost all the growth curves are likely due to a small multimode interference (MMI) effect arising from the excitation of some lower order cladding modes within the short length of phosphate fibers that are spliced between the SMF pigtails. The MMI effect not only introduces small quasi-periodic oscillations in the spectral baseline of the measured FBG transmission spectra, but also the amount of light that recombines in the core at the downstream splice at the wavelength of the grating. These two effects combine to change periodically both the amplitude and position of the Bragg resonance, but in a way that does not obscure the main trends of the UV-induced refractive index changes. The sudden drop of $\Delta \mathrm{n}_{\text {avg }}$ near the end of the curves on Figure 3.5 indicates the point where the UV irradiation is switched off. This is commonly observed in FBGs fabrication because the fiber temperature increases slightly (by about $6^{\circ} \mathrm{C}$ here, based on a measured $\mathrm{d} \lambda / \mathrm{dT}$ of $11 \mathrm{pm} /{ }^{\circ} \mathrm{C}$ ) during irradiation with high average 
power UV sources. In all sections below, the value of $\Delta \mathrm{n}_{\text {avg }}$ that are reported are the "cold" ones, as they reflect the actual values at room temperature. Unfortunately, there is no rigorous way to remove the temperature contribution from the $\Delta \mathrm{n}_{\text {avg }}$ growth curves obtained in real time but since it affects all fibers the same way, we felt it was still interesting to plot the comparative growth dynamics in Figure 3.5.
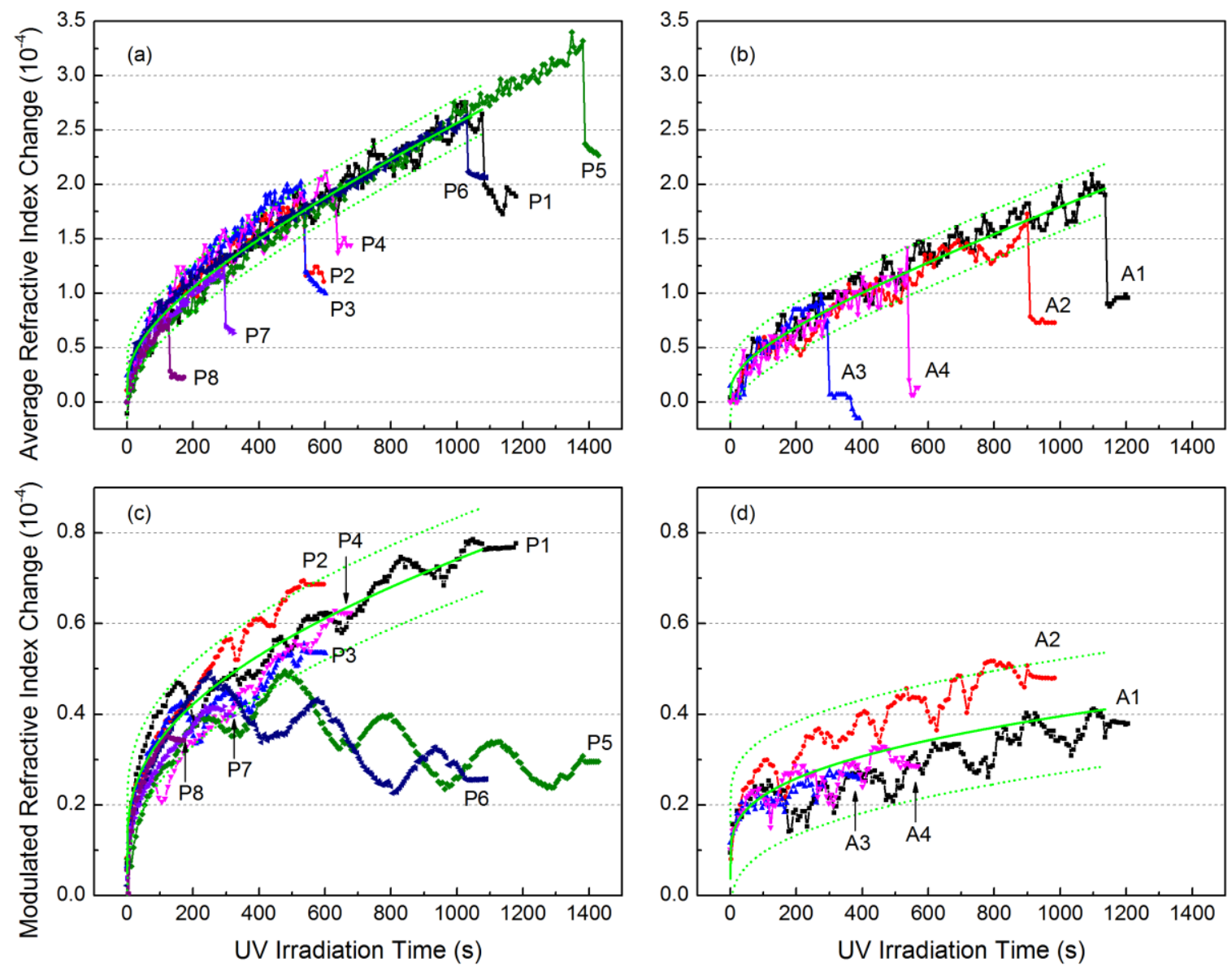

Figure 3.5 Refractive index changes during fabrication of $2 \mathrm{~cm}$-long gratings in passive and active phosphate fibers: (a) $\Delta \mathrm{n}_{\text {avg }}$ in passive fibers (samples P1-P8), (b) $\Delta \mathrm{n}_{\text {avg }}$ in active fibers (samples A1-A4), (c) $\Delta \mathrm{n}_{\bmod }$ in passive fibers and (d) $\Delta \mathrm{n}_{\bmod }$ in active fibers. Green solid and dash lines are fitted stretched exponential curves and their respective $95 \%$ prediction bands. 
The first clear observation from Figure 3.5 is that the passive phosphate fiber samples exhibit larger UV photosensitivity than the active fiber samples, evidenced by higher refractive index change in terms of both $\Delta \mathrm{n}_{\text {avg }}$ and $\Delta \mathrm{n}_{\text {mod: }}$ the passive fibers reach $\Delta \mathrm{n}_{\text {avg }}$ of $\sim 2.0 \times 10^{-4}$, while the active fibers show lower $\Delta \mathrm{n}_{\text {avg }}$ of $\sim 9.6 \times 10^{-5}$. For the grating refractive index modulation, the passive fibers reach $\Delta \mathrm{n}_{\text {mod }}$ of $\sim 7.6 \times 10^{-5}$ (corresponding to grating transmission notch depth of $\sim 14.3 \mathrm{~dB}$ ), and the active fibers only achieve a maximum $\Delta \mathrm{n}_{\text {mod }}$ of $\sim 4.8 \times 10^{-5}$ (corresponding to grating transmission notch depth of $\sim 7.2$ $\mathrm{dB})$. The lower effective photosensitivity of active fibers compared to passive ones may be due to the strong absorption of $\mathrm{Er}$ and $\mathrm{Yb}$ ions in the core at $193 \mathrm{~nm}$ [41]. We note that the grating fringe contrast ratios $\left(\Delta \mathrm{n}_{\bmod } / \Delta \mathrm{n}_{\text {avg }}\right)$ are only 0.38 and 0.40 for passive and active phosphate fibers respectively, compared to 0.67 for silica fibers (SMF28) with low-Ge doped cores that were exposed in the same setup. The difference could be due to different mechanisms for the UV-induced $\Delta \mathrm{n}_{\text {avg }}$ and $\Delta \mathrm{n}_{\text {mod. }}$.

Figure 3.5 also shows that the $\Delta \mathrm{n}_{\text {mod }}$ of gratings P5-P6 stops growing between 300 and 500 seconds of irradiation, while the $\Delta \mathrm{n}_{\text {avg }}$ follows the general trend. This indicates loss of contrast, which may be attributed to instabilities in the beam or in the mechanical set-up. Surprisingly, it will be shown below that this loss of contrast during irradiation had little effect on the subsequent thermal growth of $\Delta \mathrm{n}_{\text {mod. }}$.

To investigate the thermally induced growth dynamics and the thermal stability of UV-induced FBGs in phosphate fibers, both passive and active FBG samples were subjected to a step-isothermal annealing treatment at temperatures from $100^{\circ} \mathrm{C}$ to $300{ }^{\circ} \mathrm{C}$ in steps of $50{ }^{\circ} \mathrm{C}$. For each step of isothermal annealing, the gratings were held at the same temperature for a duration of about 9 days, after which gratings were cooled down 
to room temperature before the next annealing cycle. Figure 3.6 illustrates the thermal evolutions of the transmission notch depths of several representative gratings in both passive and active phosphate fibers. All gratings except sample P8 exhibit a growth of the transmission notch depth (i.e., $\Delta \mathrm{n}_{\text {mod }}$ increases) upon thermal annealing at temperatures of $100-250^{\circ} \mathrm{C}$. However, beyond that a thermal decay of the grating strengths begins when the annealing temperature is further increased to $300{ }^{\circ} \mathrm{C}$. The discontinuities in the curves correspond partly to a reversible grating strength change that occurs when the fibers are returned to room temperature between heating steps [63], and partly to a change in the interference pattern of the MMI due to the change in temperature. In order to minimize the impact of this artifact, an average between the measured notch depth values at the



Figure 3.6 Thermal evolution of the transmission notch depths for gratings in passive (P1, P6, P8) and active (A1, A4) phosphate fibers during step-wise annealing between 100 $300{ }^{\circ} \mathrm{C}$, in $50{ }^{\circ} \mathrm{C}$ steps for $9-11$ days at each step and return to room temperature between steps. See text for further details. 
annealing temperature and at room temperature was used to calculate the final $\Delta \mathrm{n}_{\text {mod }}$ values (labeled in Figure 3.6) at the end of the $250^{\circ} \mathrm{C}$ step.

Figure 3.7 shows the evolution of transmission spectra of one example grating P1 before and after each thermal annealing step. All spectra were measured at room temperature between heating steps. Clearly, UV induced gratings in phosphate glass fibers maintain excellent spectral quality from the initial seed grating and throughout the thermal growth process, accompanied by a blue shift due to the decay of $\Delta \mathrm{n}_{\text {avg. }}$. The linewidth of this 2-cm-long FBG increased from $0.09 \mathrm{~nm}$ to $0.19 \mathrm{~nm}$ as expected from the increase in grating strength. A similar behavior is also observed for the case of the active fiber as shown in Figure 3.8.

Before exploring further the impact of thermal annealing on $\Delta \mathrm{n}_{\text {mod }}$, its effect on $\Delta \mathrm{n}_{\mathrm{avg}}$ is illustrated in Figure 3.9. Those values are extracted from the wavelength shifts of the grating resonances measured at room temperature between each heating step. The thermally induced $\Delta \mathrm{n}_{\mathrm{avg}}$ of all the samples is plotted relative to their pre-annealing (but post-irradiation) values. In order to facilitate comparisons between gratings, the aswritten value of $\Delta \mathrm{n}_{\text {avg }}$ for each sample is included beside its label name. In contrast to the thermal behavior of $\Delta \mathrm{n}_{\text {mod }}, \Delta \mathrm{n}_{\text {avg }}$ exhibits a thermal decay for all annealing cycles and fibers, even at the relatively low heating temperature of $100{ }^{\circ} \mathrm{C}$. Furthermore, the thermal decay of $\Delta \mathrm{n}_{\mathrm{avg}}$ is almost completely independent of its initial value (and hence the UV irradiation time), as all samples fabricated in the same type of fiber experience the same decay amount. 


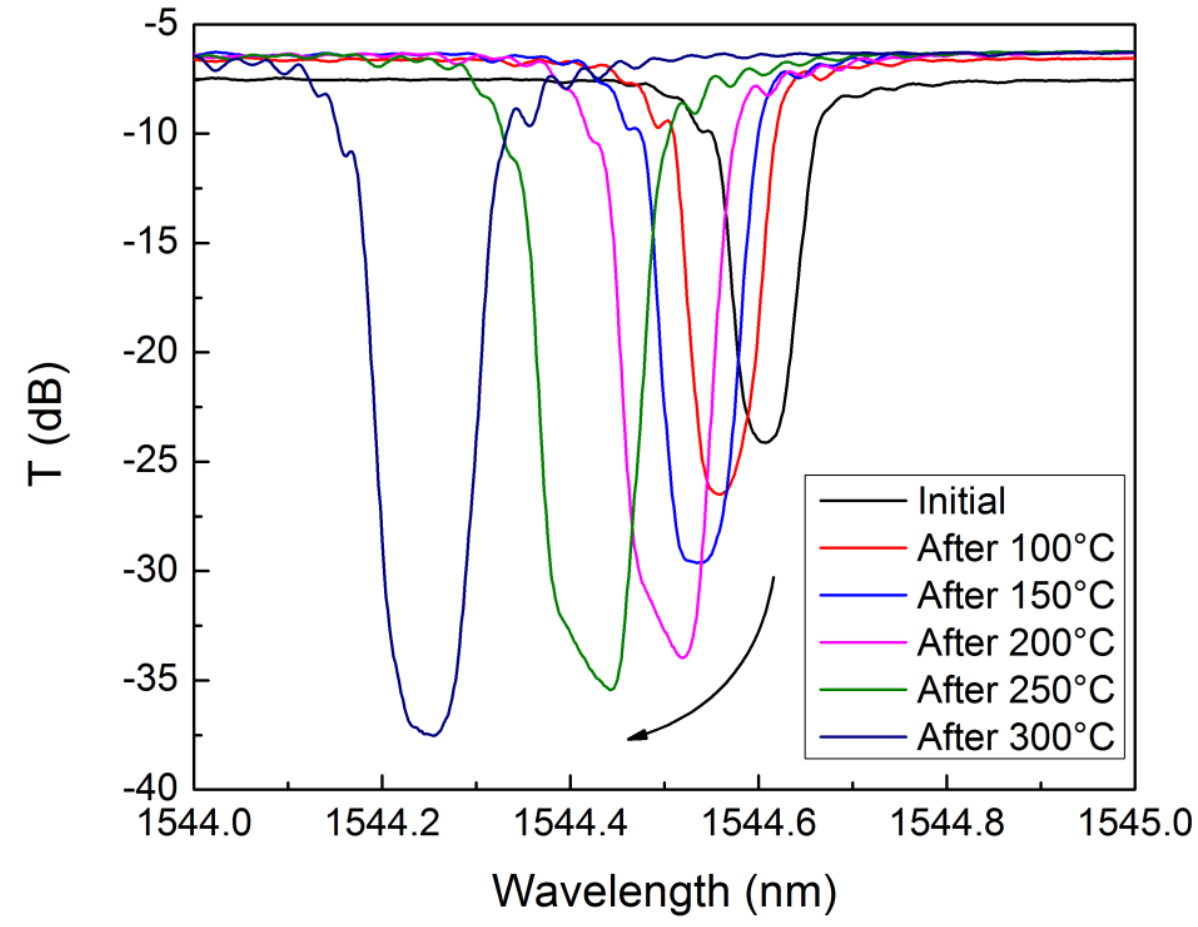

Figure 3.7 Evolution of transmission spectra of grating P1 measured at room temperature, starting from the UV induced seed and after each thermal annealing step.

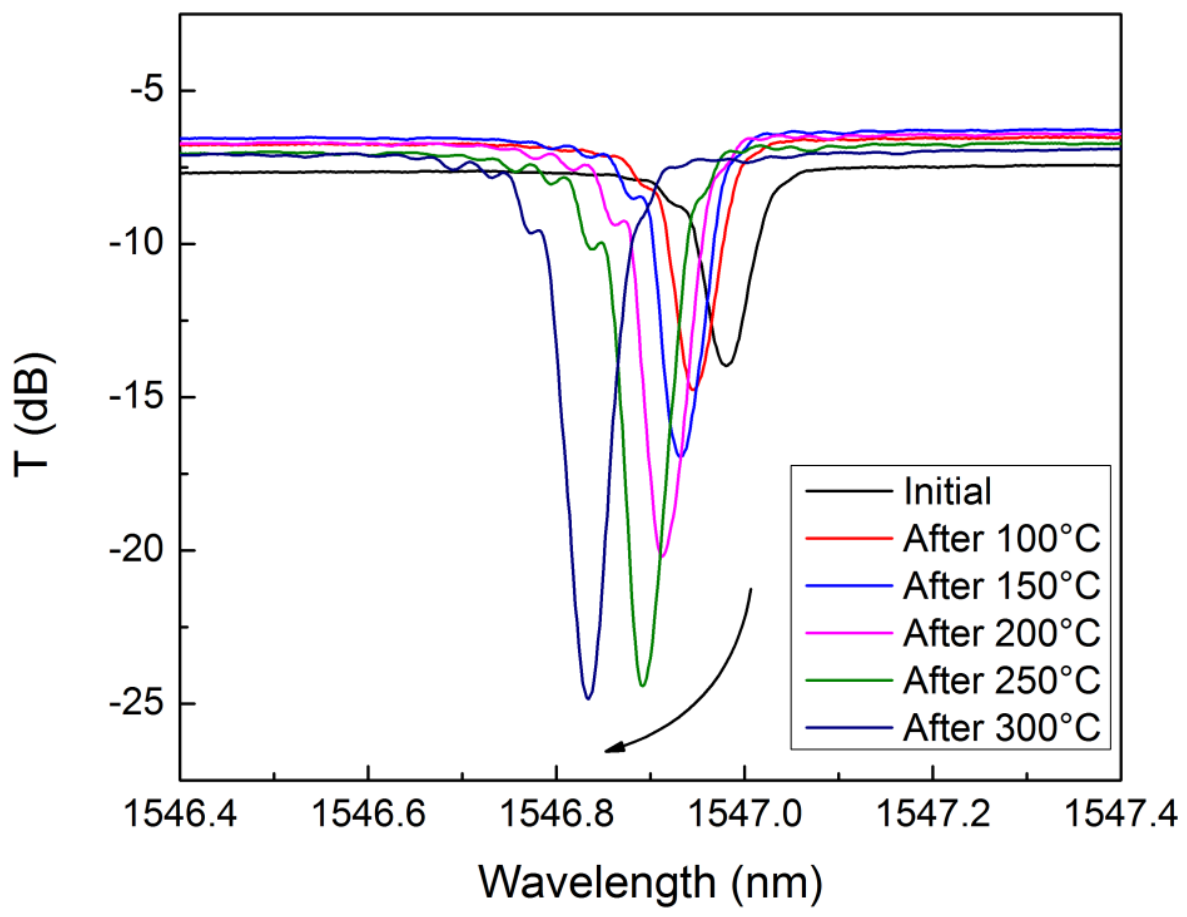

Figure 3.8 Evolution of transmission spectra of grating A1 measured at room temperature, starting from the UV induced seed and after each thermal annealing step. 


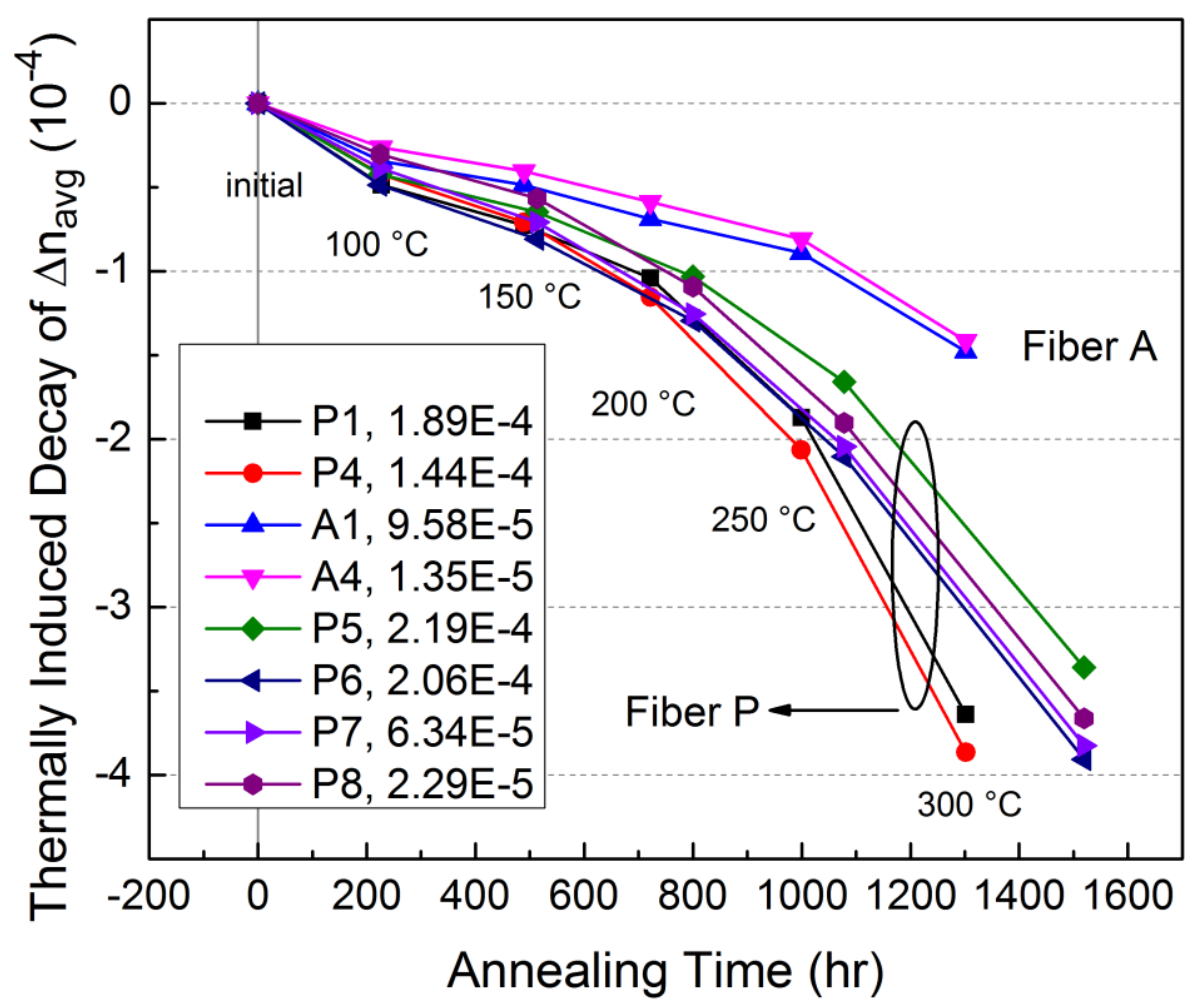

Figure 3.9 Thermally induced decay of the average refractive index change $\Delta \mathrm{n}_{\text {avg }}$ relative to its initial value for gratings in passive and active phosphate glass fibers during the annealing described in the caption of Figure 3.6. The initial absolute value of $\Delta \mathrm{n}_{\text {avg }}$ for each sample is included in the legend labels.

However, the thermal decay in the active fibers (with $\mathrm{Er} / \mathrm{Yb}$ dopants) is smaller (by about $50 \%$ ) than in passive fibers (this is the same ratio that was observed for the UV-induced growth in Figure 3.5). It is interesting to note that the thermally induced decay of $\Delta \mathrm{n}_{\mathrm{avg}}$ can be even larger than the $\Delta \mathrm{n}_{\mathrm{avg}}$ increase induced during UV illumination. Therefore, the average core refractive indices of both types of phosphate fibers can be lower than their as-drawn values following irradiation and thermal processing. The total average decay of $\Delta \mathrm{n}_{\text {avg }}$ reached after thermal processing amounts to $\sim 3.6 \times 10^{-4}$ and $\sim 1.4 \times 10^{-4}$ in passive and active fibers, respectively. Separate experiments on similar fibers have been made where the annealing treatment was performed prior to the UV 
irradiation and have shown that the core index (as measured by the starting Bragg wavelength at low fluence) is unchanged from that of un-annealed fibers.

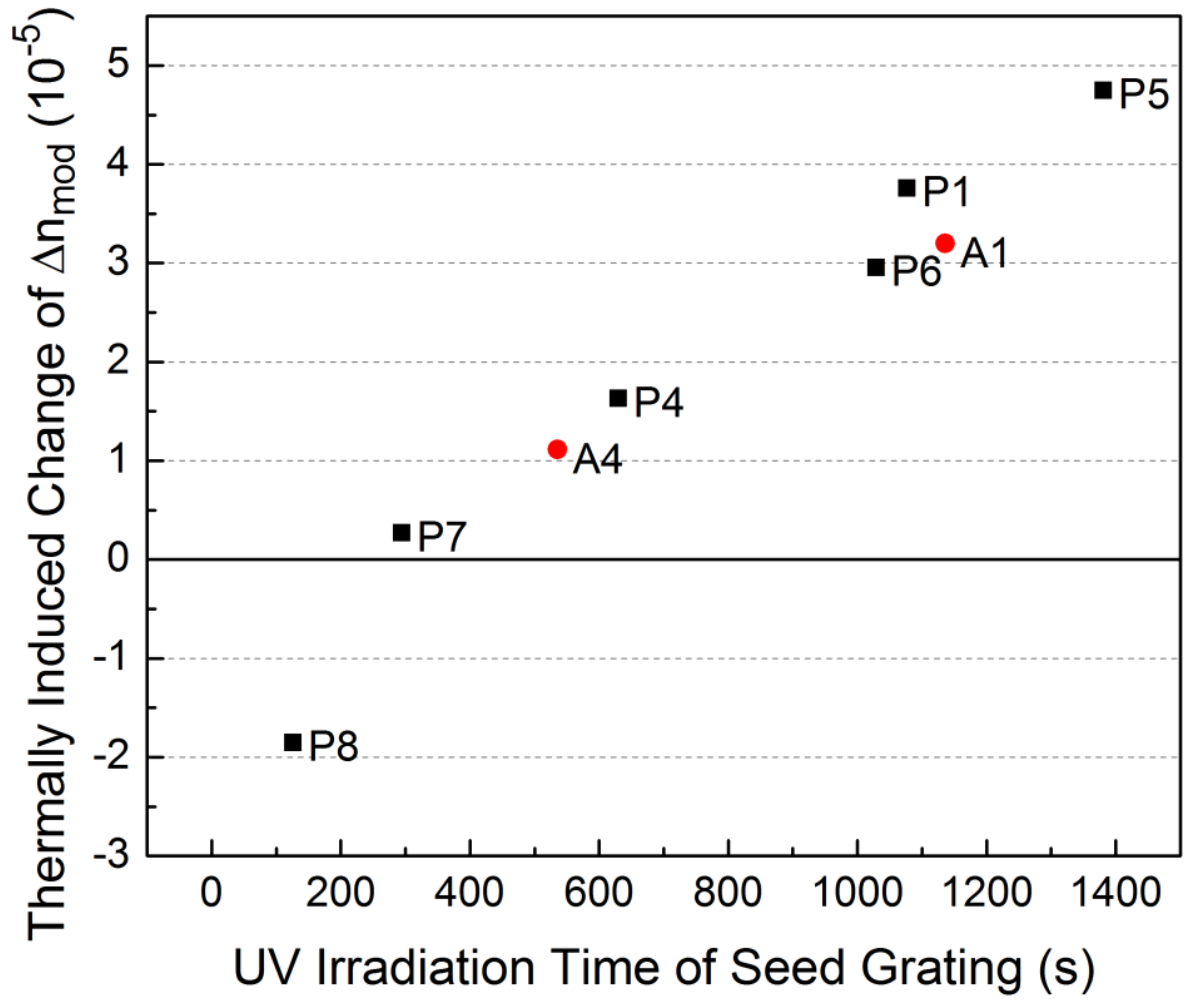

Figure 3.10 Thermally induced change of refractive index modulation $\Delta \mathrm{n}_{\text {mod }}$ at the end of the $250{ }^{\circ} \mathrm{C}$ anneal step versus the UV irradiation time applied to produce the seed gratings.

Finally, the thermally induced change of $\Delta \mathrm{n}_{\bmod }$ is plotted versus the UV irradiation time applied to inscribe the seed gratings in Figure 3.10. The thermally induced changes of $\Delta \mathrm{n}_{\text {mod }}$ presented here are the differences between $\Delta \mathrm{n}_{\text {mod }}$ after annealing at $250{ }^{\circ} \mathrm{C}$ and their respective values obtained just after UV-irradiation. The thermal decay observed at $300{ }^{\circ} \mathrm{C}$ is not considered here, as our investigation aims to find a recipe to maximize the thermal growth of $\Delta \mathrm{n}_{\text {mod. }}$. As shown in Figure 3.10, there is a clear correlation between the thermally induced change of $\Delta \mathrm{n}_{\bmod }$ and the UV irradiation time that produced the seed gratings, despite of the differences in fiber types and grating 
fabrication setup alignments. The longer the fibers were exposed to UV irradiation, the larger the thermal growth that can be achieved to maximize $\Delta \mathrm{n}_{\text {mod. }}$. However, this occurs regardless of the starting value of $\Delta \mathrm{n}_{\text {mod, }}$, as evidenced by fibers P1, P5, and P6. It is also notable that this is the only result in which there is no observable difference between passive and active fibers. Also note, for irradiation times less than about $300 \mathrm{~s}$, there is a decay of $\Delta \mathrm{n}_{\text {mod }}$ upon thermal annealing instead of a growth.

\subsection{Photosensitivity Mechanism of UV-induced FBGs in Phosphate}

\section{Fibers}

While the UV photosensitivity observed here follows well established trends (stretched exponential monotonic growth and lesser growth when some additional absorption is present, as is the case of the active fibers), the thermal behavior of the gratings is completely anomalous. Normally, the reflectivity of UV-induced gratings (i.e. $\Delta \mathrm{n}_{\text {mod }}$ ) decays with increasing temperature, in a range well below the glass transition temperature $\left(\mathrm{T}_{\mathrm{g}}\right)$ (i.e. for annealing in the $100-300{ }^{\circ} \mathrm{C}$ range for silica fibers with a $\mathrm{T}_{\mathrm{g}}$ above $1000{ }^{\circ} \mathrm{C}$ ). Here, the opposite occurs at temperatures, up to $250{ }^{\circ} \mathrm{C}$, that are quite close to the glass transition temperature of these glasses $\left(\sim 448{ }^{\circ} \mathrm{C}\right)[50]$. Furthermore, while the decay of $\Delta \mathrm{n}_{\text {avg }}$ is expected, in the current case this decay is larger than the increase that was obtained during UV-irradiation.

These observations are also different from the behavior of regenerated gratings in hydrogenated silica glass fibers [64], in which $\Delta \mathrm{n}_{\text {mod }}$ shows a complete erasure followed by a regeneration upon annealing at $\sim 900{ }^{\circ} \mathrm{C}$. Here, $\Delta \mathrm{n}_{\text {mod }}$ exhibits monotonic growth without any erasure during heat treatment. The low $\mathrm{T}_{\mathrm{g}}$ of phosphate glass, along with the 
very strong correlation between the total UV irradiation, the total amount of thermal decay of the average index, and the amount of observed thermal growth of $\Delta \mathrm{n}_{\text {mod }}$ indicate a complex photo-thermal process that may involve several index change mechanisms.

As mentioned earlier, there are few prior studies of the optical and structural properties of UV-irradiated phosphate glasses. However, several groups have used ultrafast laser techniques to permanently modify the refractive index of phosphate glasses, both for making gratings and to write waveguides in bulk samples [42-44,4749]. These references also indicate that the photosensitivity of phosphate glass is indeed a complicated process which can even generate either positive or negative index changes depending on the glass composition and laser irradiation conditions. As in the case of silica glasses [33], the photosensitivity of P-glass is thought to arise from two contributions: 1) a glass structural modification due to UV (or multiphoton) breaking of bonds in the glass network, and 2) the formation of color centers. Based on these findings, and the fact that ultrafast laser irradiation of glasses has similarities with lower intensity UV-irradiation as it relies on multiphoton effects to disrupt the electronic structure of glasses, the following phenomenological model is proposed to explain the results presented here. In this model (shown schematically in Figure 3.11), and based on generally accepted theories for the photosensitivity of glasses, high-intensity UV irradiation in the bright fringes of the interference pattern used to fabricate the grating breaks some glass network bonds (P-O bonds in this case) and induces de-polymerization of the phosphate glass network $[41,42,47]$. In our case, the result is a glass densification which occurs from the collapse of the long chain structure into a shorter-range structure. Color centers are also created in the bright fringes, with a strong absorption at $467 \mathrm{~nm}$ 
that has been assigned to phosphorus oxygen hole center as well as shorter wavelength bands assigned to other $\mathrm{P}$ centers $[54,65]$. Both of these effects increase the refractive index in the bright fringes but while the color center contribution to the index increase occurs faster, it saturates at a low level, and is thermally unstable [66]. Some index increase also occurs between the bright fringes because the interference pattern resulting from the proximity phase mask technique is not perfect and does not have $100 \%$ modulation depth. It is also well established that structural effects tend to become more thermally stable during long irradiations as the glass network increasingly deforms from its initial state.
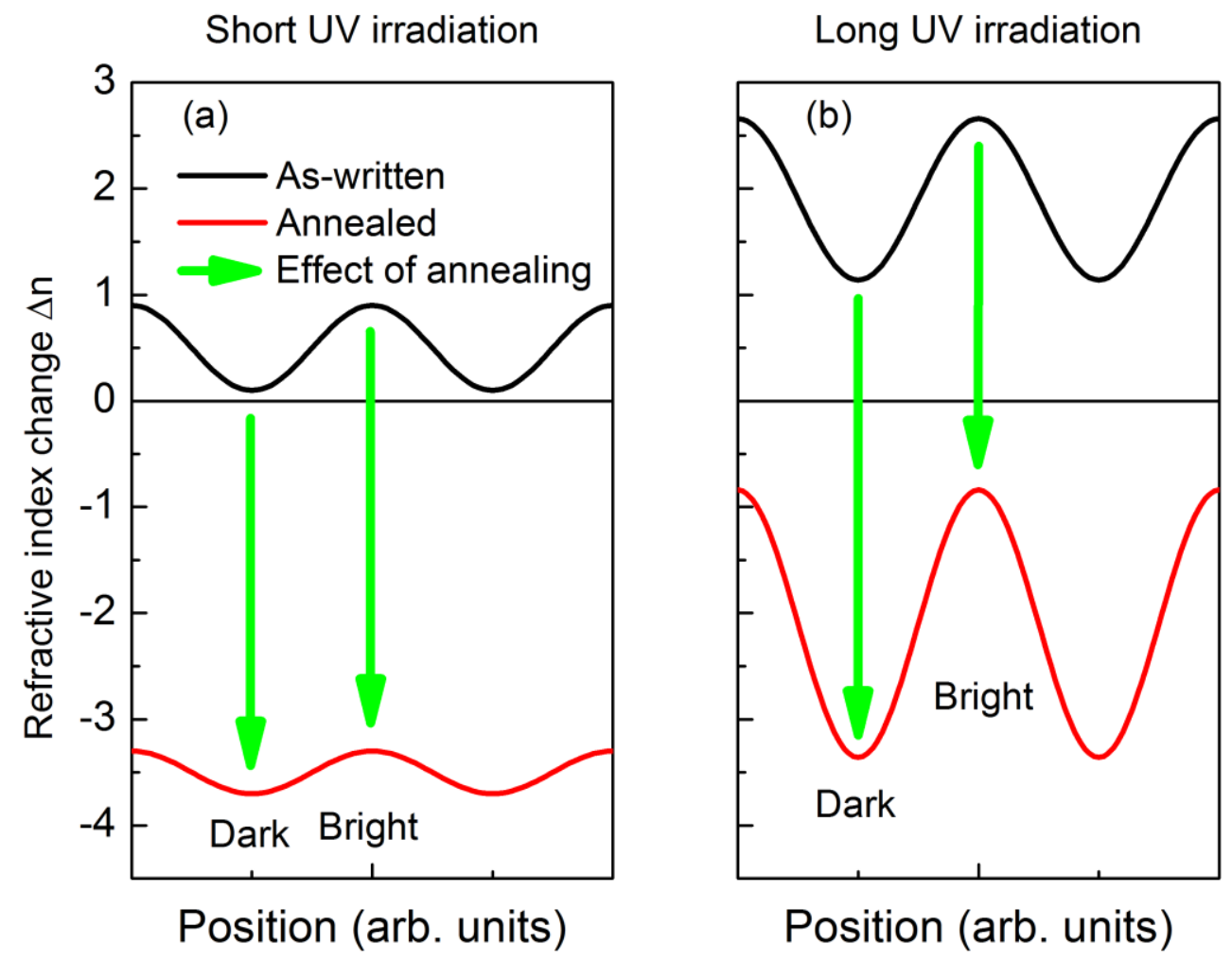

Figure 3.11 Phenomenological model of photo-induced index change (black line), thermal annealing (green arrows) and final index changes (red line) for (a) short UV irradiation and (b) long UV irradiation. See text for details. 
Based on these general considerations, our results can be explained in the following manner. UV irradiation increases the refractive index of the glass everywhere, but more strongly in the bright fringes. The result is an increase in the average index and in the index modulation, as observed in Figure 3.5. Thermal processing erases quickly the color center contribution and the more thermally unstable part of the structural changes, i.e. the index decreases more quickly in the dark fringes than in the bright fringes, resulting in an increase in the index modulation, and the difference in the thermal decay rate is larger for gratings written for longer times. The only exception to the latter process is for the case of very short irradiations, where the index increase in the bright fringes is small and mostly due to color centers and relatively unstable structural contributions, with little index change in the dark fringes. This leads to decay of the index modulation as well as of the average index (fiber P8). The main peculiarity of the P-glass system then becomes the fact that the negative shift of the average index during annealing is relatively independent of the irradiation time and appears to be larger than the amount of increase observed during the UV irradiation. We believe this to be due to the fact that the whole fiber (core and cladding) is somewhat photosensitive and therefore that the structural damage suffered during the irradiation allows the fiber to relax thermally towards a less dense state with lower average index during annealing. Micro-analysis of the physical state of the glass at various stages of processing will be required to confirm these hypotheses.

As a final comment, it is worth mentioning that it is quite a bit easier to achieve large refractive index modifications in many kinds of glasses and other materials with ultrafast laser irradiation [49]. However the current approach to achieve practically useful 
refractive index modulations in phosphate glasses from UV irradiation still has merit as it allows the writing of strong and thermally stable gratings with high spectral quality (Figure 3.7) from conventional fiber and waveguide grating fabrication tools (excimer laser and phase mask).

\subsection{Conclusion}

In conclusion, a systematic investigation of the UV photosensitivity and thermal stability of gratings written by intense $193 \mathrm{~nm}$ irradiation in both undoped and $\mathrm{Er} / \mathrm{Yb}$ doped phosphate glass fibers was performed. The conditions required to form a high reflectivity FBG in phosphate fibers through a combined photo-thermal process were determined: it basically consist of a prolonged UV irradiation (we used 20 minutes at 50 pulses per second and $46 \mathrm{~mJ} / \mathrm{cm}^{2}$ pulse fluence at the fiber) with some modulation of the UV light pattern to form a seed grating, followed by gradual heating up to $250{ }^{\circ} \mathrm{C}$. Under these conditions, which are the same for undoped and doped fibers, were shown to lead to strong refractive index modulation with $\Delta \mathrm{n}_{\text {mod }} \sim 1.3 \times 10^{-4}$ in undoped fibers and $8.4 \times 10^{-5}$ in doped fibers. Further annealing at $300{ }^{\circ} \mathrm{C}$ reverses the process and induces the beginning of a decay in $\Delta \mathrm{n}_{\text {mod. }}$. Over the whole annealing process, a significant decay of the average core refractive index change $\Delta \mathrm{n}_{\text {avg }}$ is observed in all fibers at all annealing temperatures, leading to a net negative $\Delta \mathrm{n}_{\text {avg }}$ (up to $-1 \times 10^{-4}$ ) relative to the value of as drawn core refractive indices. With regards to the long term thermal stability of these gratings, the fact that heating to $250^{\circ} \mathrm{C}$ for as long as 9 days does not lead to $\Delta \mathrm{n}_{\text {mod }}$ decay is a very encouraging feature. 


\section{Chapter 4}

\section{Photodarkening in Phosphate Glass Fibers}

As discovered in Chapter 3, the inscription of UV-induced fiber Bragg gratings in phosphate glass fibers is accompanied by an additional light-induced excess loss as high as $\sim 1 \mathrm{~dB} / \mathrm{cm}$ across $\mathrm{C}$-band. Because it is the operational wavelength of $\mathrm{Er} / \mathrm{Yb}$ doped phosphate fiber lasers, this remarkable loss will deteriorate the lasing performance of the UV-induced gratings-based laser cavity. In this chapter, a detailed investigation of this deep UV-induced photodarkening (PD) effect in phosphate fibers is performed in both active and passive fiber samples. The broadband absorption spectra from VIS to NIR of darkening samples are characterized with the use of a supercontinuum source. The origin of the photodarkening effect is found to be related to the formation of a phosphorusrelated color center with an absorption band at $467 \mathrm{~nm}$. Photo-bleaching and thermobleaching processes are demonstrated as both being effective for the complete erasure of PD loss while not diminishing the reflectivity of UV-induced FBGs. The work in this chapter has been published in one paper [65].

\subsection{Introduction}

Rare-earth-doped phosphate glass (without silica) is a popular medium for high power amplifiers and compact fiber lasers because of the higher solubility of rare earth elements relative to silica glass [2,4]. An additional benefit of this material is the absence of pump-induced photodarkening (PD) at near infrared (NIR) wavelengths [67]. In this 
chapter, detailed results are presented about a significant NIR PD caused by short wavelength ultraviolet irradiation at $193 \mathrm{~nm}$ used to form Bragg grating laser mirrors in phosphate fibers and about ways to eliminate this PD.

The PD effect, referring to light irradiation induced absorption, has been studied in many kinds of rare-earth doped optical fibers $[68,69]$, mostly in Yb-doped silica and phospho-silica optical fibers, because the NIR darkening loss induced by high-power pumping severely degrades the output power and long-term reliability of high-power fiber lasers [70-73]. In addition to PD induced by pump light at $980 \mathrm{~nm}$, PD was also observed to occur under irradiation at 193 and $488 \mathrm{~nm}[74,75]$. Although the exact mechanism of the PD in $\mathrm{Yb}$ doped silica fiber is still under debate, the light induced loss is commonly attributed to the formation of color centers by photo-ionization via either single- or multi-photon absorption processes depending on the irradiation wavelength [70,75-78]. The peak absorption wavelength observed in different glasses depends on the species of color centers generated and ranges from the visible (VIS) to the ultraviolet (UV). Invariably, all color center absorption bands show a long tail extending into the near-infrared (NIR), and hence a noticeable loss is generated at the pump and signal wavelengths of $\mathrm{Yb}$-doped fiber lasers. In all these silica glasses, however, the absorption decreases gradually further in the NIR and becomes negligible in the $1550 \mathrm{~nm}$ telecommunication window. Hence, the PD effect has not been considered detrimental to Er doped fiber lasers operating around $1550 \mathrm{~nm}$ even with $\mathrm{Yb}$ co-dopants in the fiber.

For higher power, shorter length fiber lasers made from phosphate glass with integrated Bragg grating mirrors, however, the situation is different. Since phosphate fibers lack photosensitivity in the $244 \mathrm{~nm}$ spectral range commonly used to form fiber 
Bragg gratings, irradiation with intense $193 \mathrm{~nm}$ light from excimer lasers is required [12].

In this chapter, it is shown that such irradiation, even over short lengths of a few $\mathrm{cm}$, causes broadband absorption on the order of $1 \mathrm{~dB} / \mathrm{cm}$ across the whole NIR spectrum up to the C-band. This amount of loss is sufficient to be detrimental to grating based

phosphate fiber laser performance at these wavelengths [24]. Hence the PD induced by $193 \mathrm{~nm}$ light is an important problem to address. The only reported work on the PD effect in phosphate glass fibers is that of Y.W. Lee et al. [67]. They found that, even with higher doping concentrations of $\mathrm{Yb}$, the phosphate fiber showed greatly reduced PD loss compared to silica fiber when pumped at $980 \mathrm{~nm}$. Here however, a significant PD in both Er/Yb-doped and undoped phosphate glass fibers under intense $193 \mathrm{~nm}$ irradiation is reported. It is further reported how this PD can be removed completely through both photo- and thermal- bleaching processes.

\subsection{Photodarkening in Phosphate Glass Fibers}

The PD experiments were performed on three types of fiber samples made from the same base glass composition (a silica-free phosphate glass similar to the one described in [50], see detailed composition provided in Section 2.4.2 of this work): Er/Yb co-doped phosphate fiber, undoped phosphate fiber, and pre-annealed doped phosphate fiber. The parameters of our fiber samples are summarized in Table 4.1. The pre-annealed fiber is the same fiber as $\mathrm{Er} / \mathrm{Yb}$ co-doped fiber, but has been annealed at $250{ }^{\circ} \mathrm{C}$ for 36 hours prior to the PD experiments. This process is aimed at finding out whether the UV induced $\mathrm{PD}$ requires precursors that can be thermally erased in advance. One point worthy of note 
is that Fiber $\mathrm{A}$ refers to one different type of active fibers (1\%-2\% $\mathrm{Er} / \mathrm{Yb}$ doped fiber) instead of the active fiber ( $1 \%-8 \% \mathrm{Er} / \mathrm{Yb}$ doped fiber) used in Chapter 3. From now on, this active fiber will be used because it allows core-pumped fiber lasers over multicentimeter length.

\begin{tabular}{|c|c|c|c|}
\hline \multirow{2}{*}{ Fiber Type } & \multicolumn{2}{|c|}{ Core Dopants } & \multirow{2}{*}{} \\
\cline { 2 - 3 } & $\begin{array}{c}\mathrm{Er}^{3+} \\
\left(\mathrm{ions} / \mathrm{m}^{3}\right)\end{array}$ & $\begin{array}{c}\mathrm{Yb}^{3+} \\
\left(\text { ions } / \mathrm{m}^{3}\right)\end{array}$ & Pre-annealed \\
\hline $\mathrm{A}$ & $1.1 \times 10^{26}$ & $2.15 \times 10^{26}$ & No \\
\hline $\mathrm{P}$ & None & None & No \\
\hline AP & $1.1 \times 10^{26}$ & $2.15 \times 10^{26}$ & Yes \\
\hline
\end{tabular}

Table 4.1 Details of phosphate fibers used for photodarkening test.

Samples of 5-cm-long, phosphate glass fibers are spliced to standard single mode (CORNING SMF 28) silica fibers and side exposed to a high intensity UV beam from a pulsed $193 \mathrm{~nm}$ ArF excimer laser (GSI Lumonics PulseMaster 840 series) through a phase mask in order to have the same irradiation conditions as for writing Bragg gratings. The laser delivered pulses with duration of $\sim 20 \mathrm{~ns}$ at a repetition rate of $100 \mathrm{~Hz}$, and the fluence per pulse at the fiber position is approximately $45 \mathrm{~mJ} / \mathrm{cm}^{2}$. Irradiation lengths of $2 \mathrm{~cm}$ are used on the samples, and the resulting UV-induced PD loss over the VIS - NIR spectral bands is measured by using a supercontinuum source with an emission from 500 $\mathrm{nm}$ to $2400 \mathrm{~nm}$ (NKT Photonics SuperK Compact) and 2 Optical Spectrum Analyzers (OSA1: Yokogawa AQ 6317B, wavelength range: $600-1750 \mathrm{~nm}, \mathrm{OSA}$ : Yokogawa AQ 6373, wavelength range: $350-1200 \mathrm{~nm}$ ). The experimental setup for in-situ measuring the photodarkening loss spectra of phosphate fiber samples during UV irradiation is illustrated in Figure 4.1. 


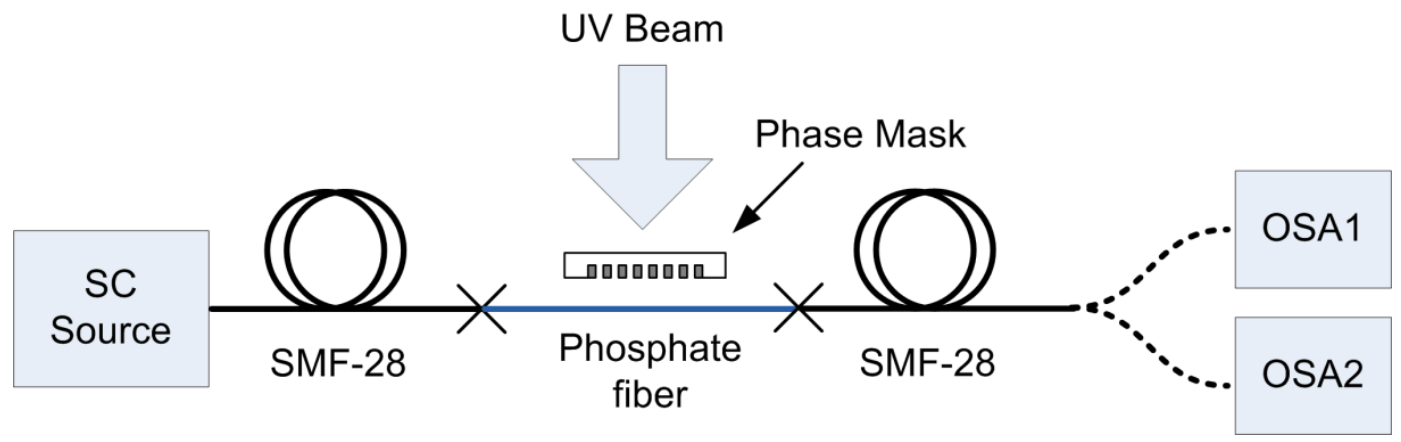

Figure 4.1 Experimental setup for in-situ characterization of photodarkening loss in phosphate fibers.

The UV-induced PD spectra of six different phosphate fibers after 500 seconds of exposure are demonstrated in Figure 4.2. The transmission spectra are normalized to the transmission of pristine fiber samples before exposure. The spectra from OSA1 and OSA2 overlap in the common spectral range $600-920 \mathrm{~nm}$, and hence are used together to show spectra over full range from VIS to NIR.

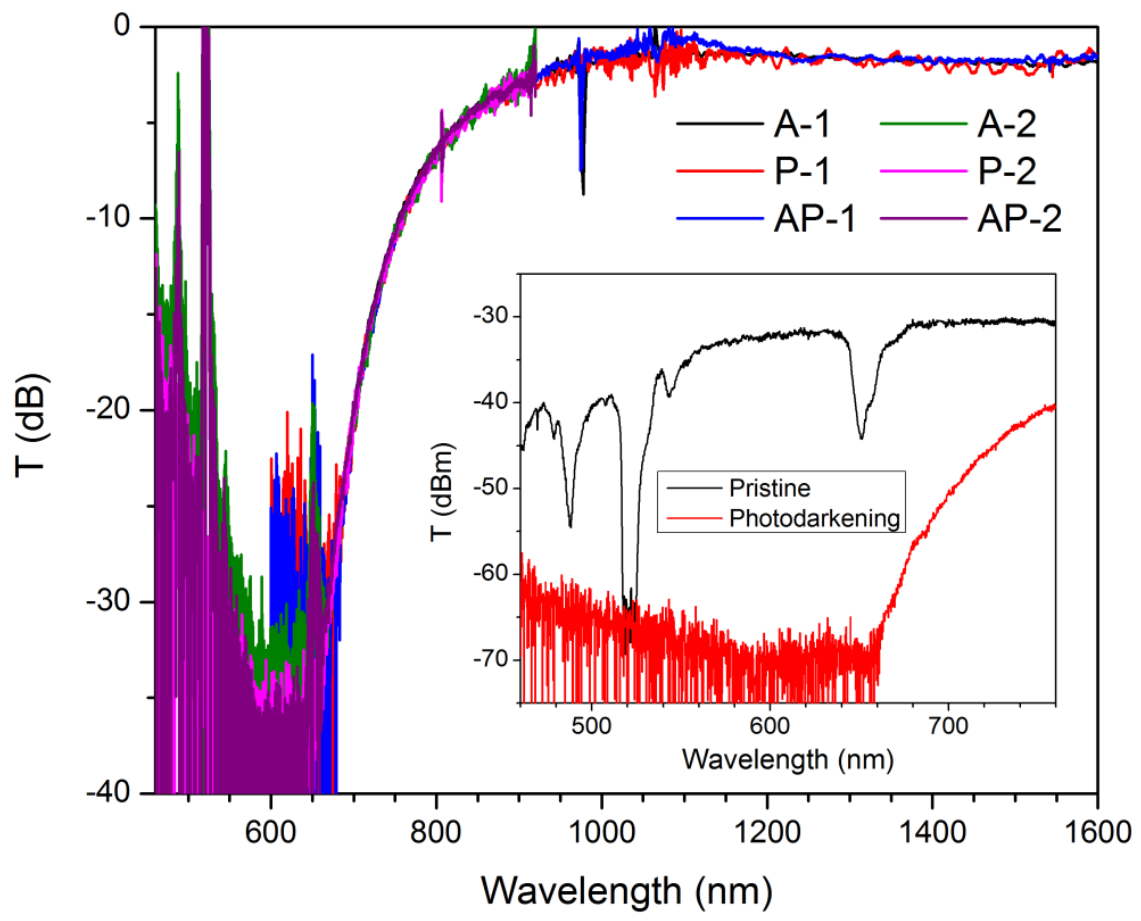

Figure 4.2 Transmission spectra of photodarkened phosphate fiber samples after $193 \mathrm{~nm}$ irradiation (Samples A-1, P-1, and AP-1 are measured with OSA1, and Samples A-2, P-2, and AP-2 are with OSA2). The inset shows the transmitted intensity spectra of a sample of Fiber A before and after UV irradiation. 
As seen in the Figure 4.2, both doped and undoped phosphate fibers, as well as the pre-annealed doped fibers all exhibit almost identical PD responses to UV irradiation. This suggests that the UV-induced PD in phosphate fibers is not due to the presence of the Er and $\mathrm{Yb}$ dopants. Also, the pre-annealing process has no impact on the PD, which is therefore not due to pre-existing, thermally erasable defects in phosphate glass (the high energy of $193 \mathrm{~nm}$ photons is sufficient to photo-ionize electrons in such glass). The absorption is much higher than that reported previously for longer wavelength irradiation in various kinds of rare-earth doped fibers [70,75]. The absorption reaches $\sim 30 \mathrm{~dB}$ (i.e. $\sim 15 \mathrm{~dB}$ per $\mathrm{cm}$ of UV-exposed fiber) at $670 \mathrm{~nm}$, and the broadband NIR loss including the C-band is $\sim 2 \mathrm{~dB}$ (i.e. $\sim 1 \mathrm{~dB} / \mathrm{cm}$ ). This significant C-band loss has not been observed in PD experiments with VIS or NIR irradiation but clearly appears under $193 \mathrm{~nm}$ [74]. The fact that the transmission at wavelengths shorter than $670 \mathrm{~nm}$ falls into the noise is truly due to darkening (and not to insufficient signal of the measurement system) as indicated by the pre- and post-irradiation measurement of a sample of fiber A shown in the inset of Figure 4.2. It should be noted that the three deep transmission dips in the spectrum of the pristine active sample correspond to the ground state absorption bands of Er ions.

In order to visualize the spectral shape of the darkening loss further in the VIS, the attenuation spectrum of the active fiber sample exposed to only 100 pulses is shown in Figure 4.3. The UV-induced absorption band fits perfectly with a single Gaussian function centered at $467 \mathrm{~nm}$. 


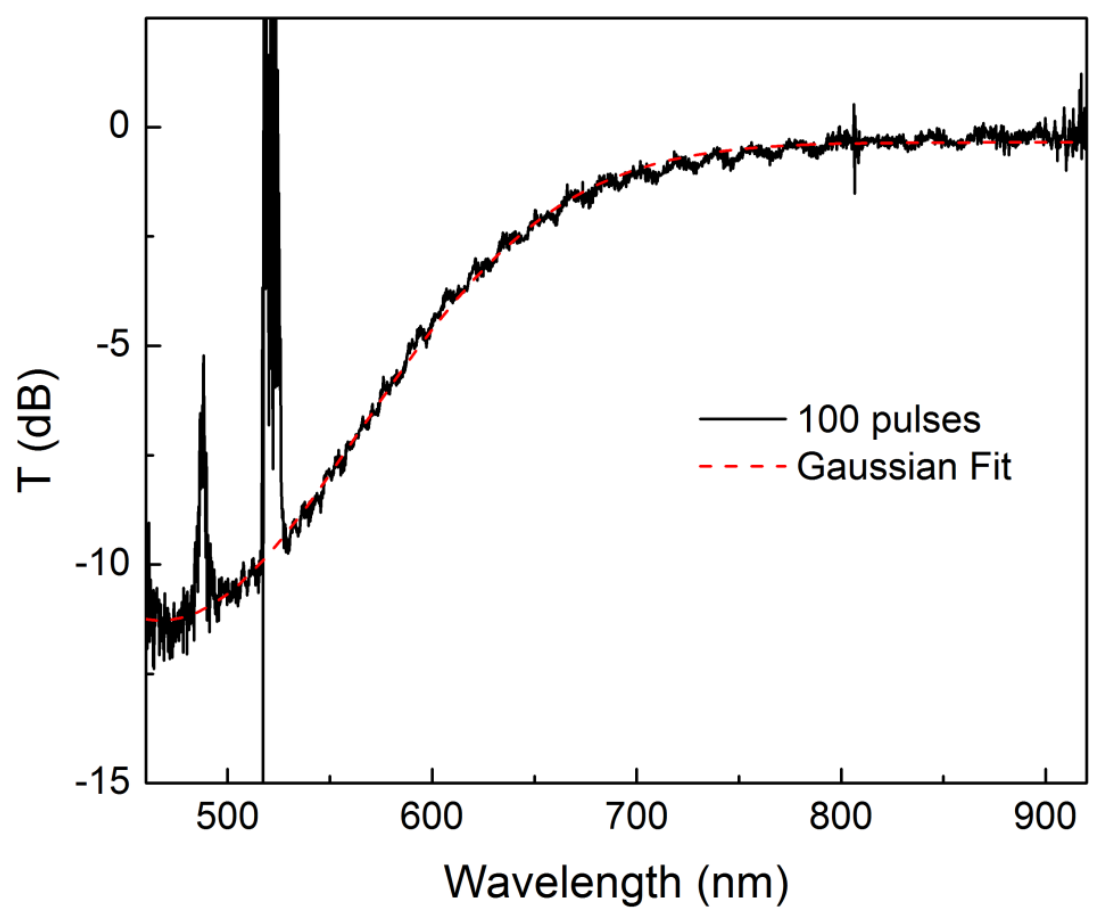

Figure 4.3 Photodarkening loss spectrum of Fiber A upon 100 pulses exposure, and the Gaussian fitting.

\subsection{Photo- and Thermo-bleaching of PD Loss in Phosphate Glass Fibers}

Right after UV irradiation, the three kinds of photodarkened fiber samples are subjected to a photo-bleaching process by leaving them connected to the white light source used for the transmission measurement. The average spectral power density launched in the fiber samples is $\sim 4.5 \mu \mathrm{W} / \mathrm{nm}$. The transmission spectra of the fiber samples are monitored continuously during the process and behave similarly. Figure 4.4 shows three representative spectra (for Fiber A): before, after 9 min, and after 40 minutes of photobleaching by the supercontinuum light. After $40 \mathrm{~min}$, the transmission of the fiber is completely restored at wavelengths longer than $800 \mathrm{~nm}$. In order to highlight the process dynamics, Figure 4.5 shows how typical samples of the three kinds of fiber darken and 


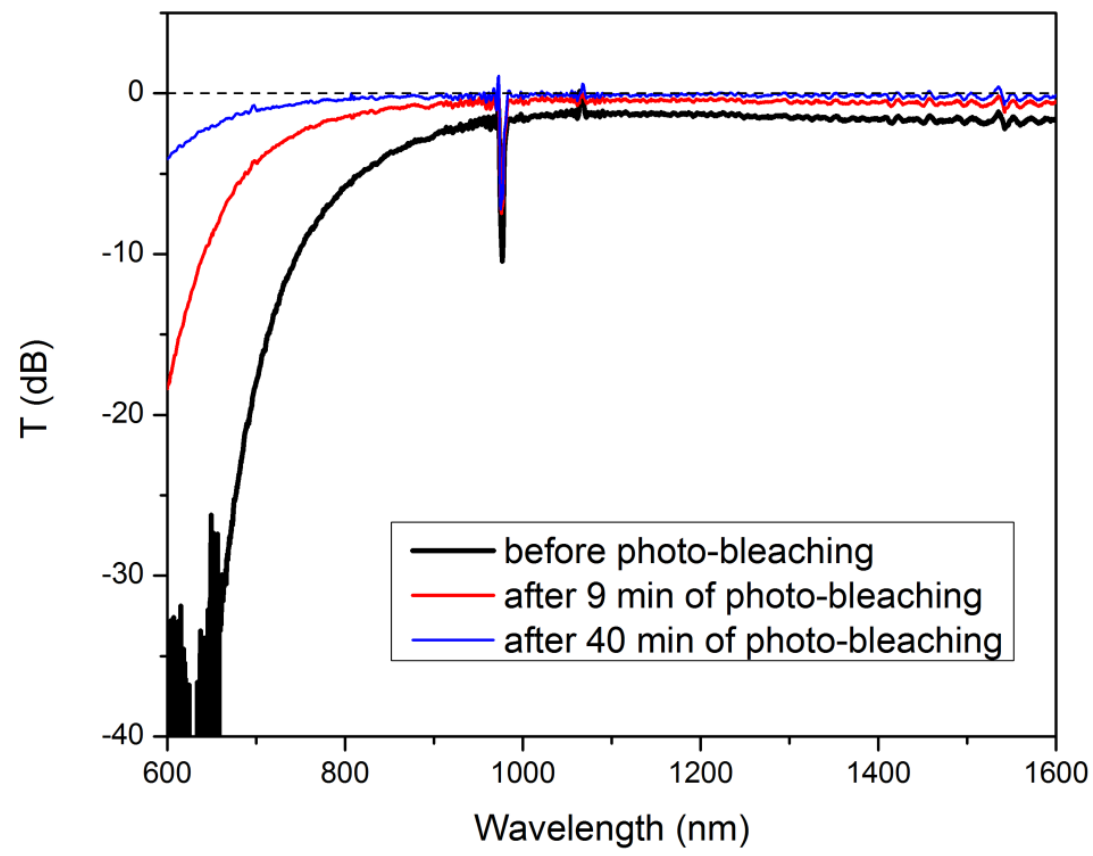

Figure 4.4. Transmission spectrum of the photodarkened Fiber A before, during (9 min) and after 40 mins of photo-bleaching.

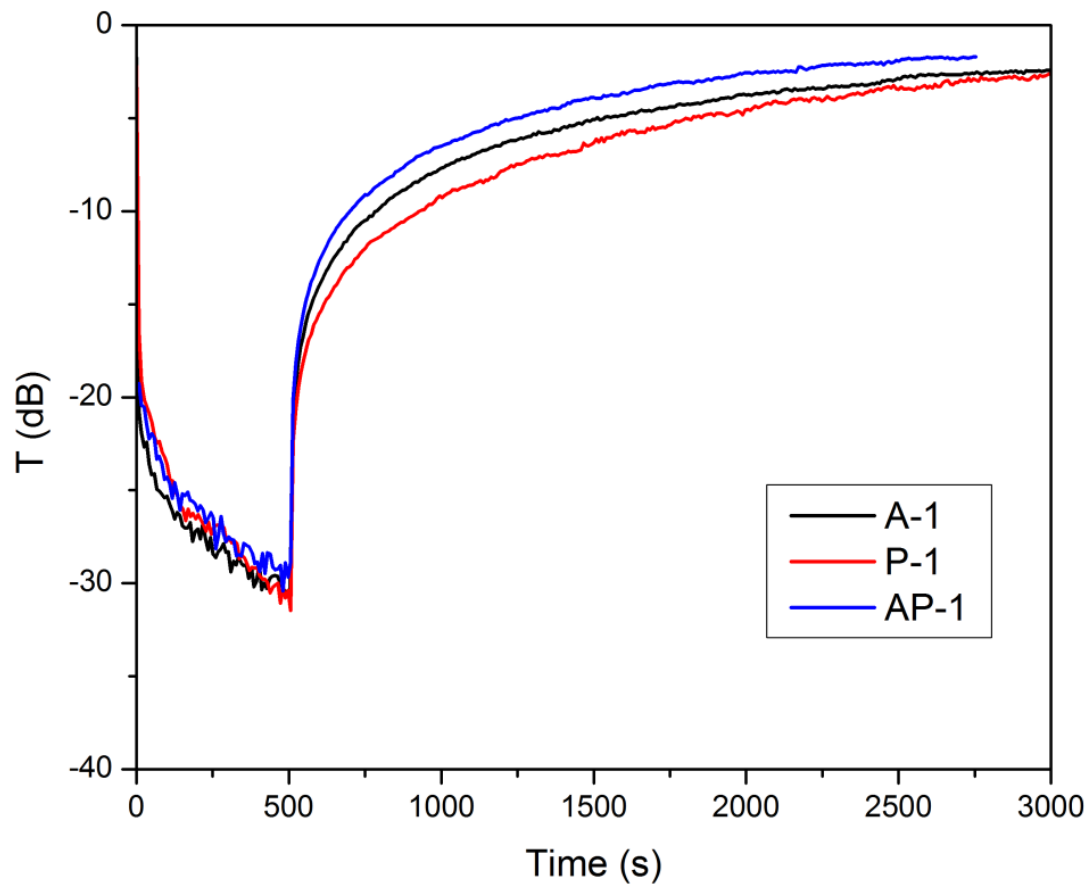

Figure 4.5 The evolution of photodarkening loss at $670 \mathrm{~nm}$ for phosphate fiber samples during the photodarkening and photo-bleaching processes. 
recover under constant UV irradiation followed by constant supercontinuum bleaching. The small difference in the bleaching rates of the samples is caused by changes in the coupled bleaching power into the fibers. We observed that the darkening losses at different wavelengths remain proportional at all times, which points to a PD that would be due to a single species of color center for this particular band. It is important to note that without photo-bleaching we believe that the PD is permanent at room temperature. We measured the PD loss over several days at room temperature, and found no decrease apart from some bleaching that occurs every time a spectrum is taken with the supercontinuum source. This photo-bleaching is only due to the VIS part of the supercontinuum: separate experiments with the VIS light filtered out by NKT Photonics SuperK SPLIT show that no bleaching occurs when the darkened sample is irradiated only by the NIR light from 1000 to $2400 \mathrm{~nm}$ (which includes a strong residual pump light at $1064 \mathrm{~nm})$.

Another investigation concerns thermal bleaching of the PD loss, which is performed on a hot plate. The plate temperature was ramped from room temperature to $200{ }^{\circ} \mathrm{C}$ over 1 hour (3600 s) after which the heat was turned off to allow the fiber to cool down. In order to avoid corrupting the thermal bleaching by photo-bleaching, only the NIR loss was monitored, using a C-band ASE source and OSA1. As we verified over a period of 4 days of continuous measurement with the ASE source, its power level is too low, and its wavelength too long, to induce photo-bleaching. The evolution of the NIR darkening losses during the thermal treatment was similar for the three kinds of fibers and a representative result for Fiber A is plotted in Figure 4.6. The annealing temperature profile is plotted schematically at the bottom of the figure. The loss in that figure is 
obtained by averaging the nearly flat spectral response over the whole C-band. The loss completely recovers during the first hour, over which the temperature reached $192{ }^{\circ} \mathrm{C}$. Separate experiments with the supercontinuum source further confirmed that the whole color center absorption band recovers during the process (inset of Figure 4.6).

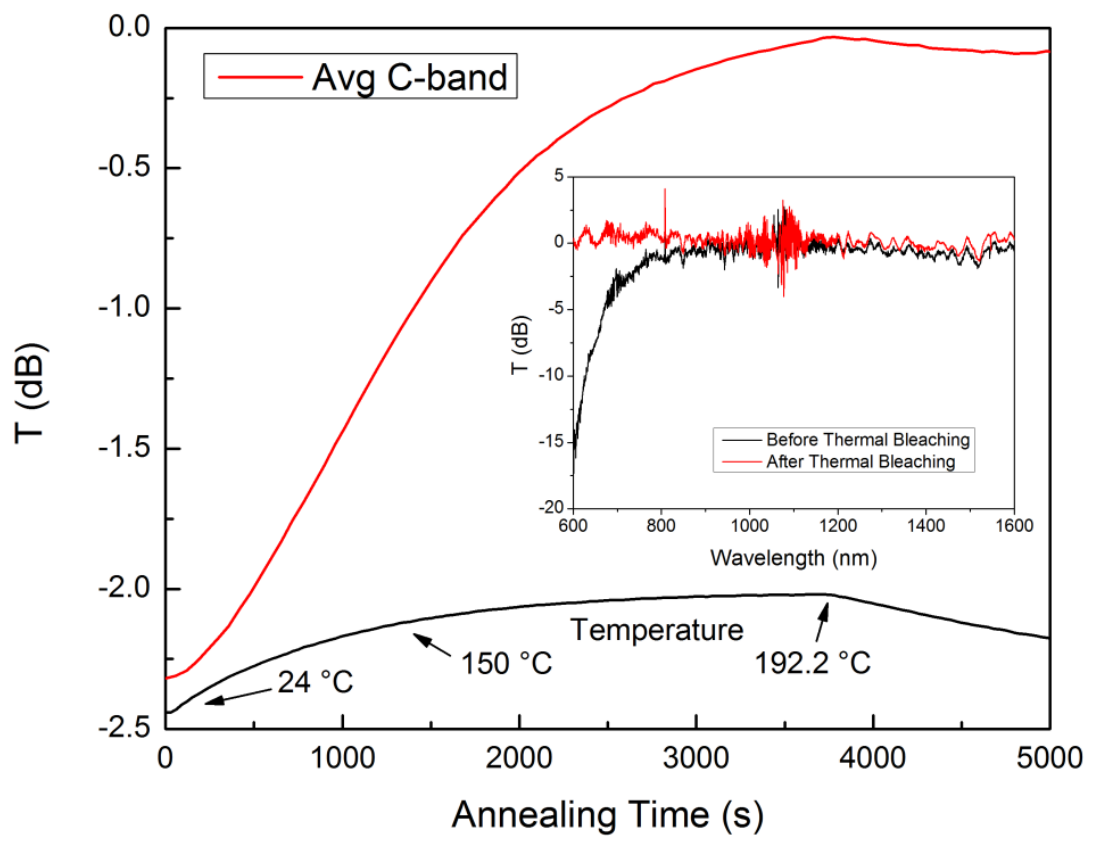

Figure 4.6 The evolution of the average photodarkening loss across C-band of Fiber A during the thermal bleaching. The inset shows the VIS-NIR transmission spectra (normalized by the transmission of the prestine sample) of a fiber sample before and after thermal bleaching. The actual temperature profile is also shown.

\subsection{Discussion of the Photodarkening Mechanism}

It is clear from our results that $\mathrm{Er}$ and $\mathrm{Yb}$ dopants are not responsible for PD under 193 $\mathrm{nm}$ irradiation in these phosphate glasses. Over the wavelength range we can measure, a single band of nearly Gaussian shape and peak wavelength $(467 \mathrm{~nm})$ was observed, in close agreement to the only previous report of PD in bulk $\mathrm{Yb}$-doped phosphate glasses induced by $266 \mathrm{~nm}$ irradiation (peak darkening at $490 \mathrm{~nm}$ ) [67]. The choice of a Gaussian to fit the observed PD was just made for convenience (and good fit), allowing 
us to pinpoint the band center but without implication on the physical origin of the defect band. Because of the similarity of the position of the absorption band, we tentatively conclude that the mechanism proposed in Ref. [67] is the same here, namely that the color centers arise from UV photons that ionize existing precursors in the fibre host material and generate free holes and electrons, which are trapped at defects in the glass matrix. This mechanism is also supported by the fact that shorter wavelength photons induce a much stronger defect band. Without further corroborative evidence from other diagnostic techniques, we cannot assign a particular defect state to the observed induced optical absorption band. However, the position of this absorption band is reminiscent of a phosphorus oxygen hole center (POHC) associated group of bands that are often observed in other kinds of glasses containing large amounts of phosphorus [54,79], as described in Section 2.4.3.

Fortunately, this significant PD loss is shown to be fully erased by photo- or thermal- bleaching processes. What is particularly useful from a practical point of view is that for the thermal bleaching process temperatures and durations used here, the reflectivity of FBGs written in these fibers using the exact same irradiation conditions actually gets stronger as demonstrated previously [12]. This clearly indicates that the photodarkening and the majority of the photo-induced refractive index change have different physical origins and that removing photodarkening does not simultaneously diminish the reflectivity of FBGs. While PD and its bleaching must be accompanied by refractive index changes (through the Kramers-Kronig relations), these changes appear negligible relative to the index change (a sinusoidal refractive index variation with amplitude of 0.0001) associated with the Bragg mirrors under similar irradiation 
conditions, according to our observation. It is also interesting to note that somewhat similar results (thermal annealing completely erases the UV induced PD but not affecting the reflectivity of UV-induced FBGs) were obtained recently in the alumino-silicate glass system, although for different irradiation and thermal regimes [80]. It is also worth pointing out that the presence of supercontinuum light in the fibers during the UV irradiation is not thought to have a significant increasing or decreasing impact on the PD itself, because earlier Bragg grating writing experiments had similar PD without the presence of monitoring light in the fiber.

\subsection{Conclusion}

In conclusion, a significant photodarkening effect has been observed in $\mathrm{Er} / \mathrm{Yb}$ doped and undoped phosphate fibers after $193 \mathrm{~nm}$ irradiation. A color center absorption band was measured with a peak near $467 \mathrm{~nm}$ and a long tail into the NIR that creates loss of the order of $1 \mathrm{~dB} / \mathrm{cm}$ across the $\mathrm{C}$-band. Fortunately the photodarkening is quite unstable and both photo- and thermal-bleaching processes have been demonstrated to be effective in erasing the darkening loss under conditions where the grating strength of the UV-induced FBG is not diminished. 


\section{Chapter 5}

\section{Single-Wavelength Single-Longitudinal-Mode}

\section{DFB Phosphate Fiber Laser}

The success of direct-writing strong gratings with ArF laser makes it feasible to build short monolithic all-phosphate fiber lasers. This chapter demonstrates the application of direct-written Bragg gratings in building a distributed feedback (DFB) fiber laser. Short DFB grating structures are inscribed into the $\mathrm{Er} / \mathrm{Yb}$ co-doped phosphate fiber, and low noise single-wavelength narrow-linewidth laser emission at C-band is achieved.

\subsection{Single-wavelength DFB phosphate fiber laser}

A 3.5-cm-long phase-shift phase mask is used to inscribe a laser cavity into the $\mathrm{Er} / \mathrm{Yb}$ codoped phosphate glass fibers. The grating pitch of the phase mask is $982.0 \mathrm{~nm}$. The phase mask is a uniform mask with a $50 \mu \mathrm{m}$ gap near the middle but off the center, which splits the mask into $2 \mathrm{~cm}$ and $1.5 \mathrm{~cm}$ long uniform sections. According to the grating reflectivity formula given by Eq. (2.4), the 2-cm-long FBG has a higher reflectivity than the 1.5-cm-long FBG under the condition of both having the same refractive index modulation. Consequently, this design ensures that the output power is predominantly emitted from one end of the DFB laser. The DFB grating structure is replicated into the active phosphate fibers during UV irradiation.

Figure 5.1 illustrates the schematic of the single-wavelength DFB phosphate fiber laser. A 5-cm-long Er/Yb co-doped (Er: 1wt\% and $\mathrm{Yb}: 2 \mathrm{wt} \%$ ) phosphate fiber is used as 
the gain medium and is spliced to standard telecommunication fiber pigtails. The doped phosphate fiber is core-pumped by a $980 \mathrm{~nm}$ laser diode through a WDM coupler. The 3.5-cm-long DFB grating structure is fabricated close to the pump launching end of the 5cm-long doped phosphate fiber, and the rear part of the doped fiber will absorb the remaining pump light and amplify the laser signal. The $2-\mathrm{cm}$ and $1.5-\mathrm{cm}$ uniform FBG1 and FBG2 of the DFB structure are the high reflectivity (HR) and low reflectivity (LR) mirrors of the laser resonator, and the DFB laser emits from the low-reflectivity FBG2 in the same direction as the pump light propagation.

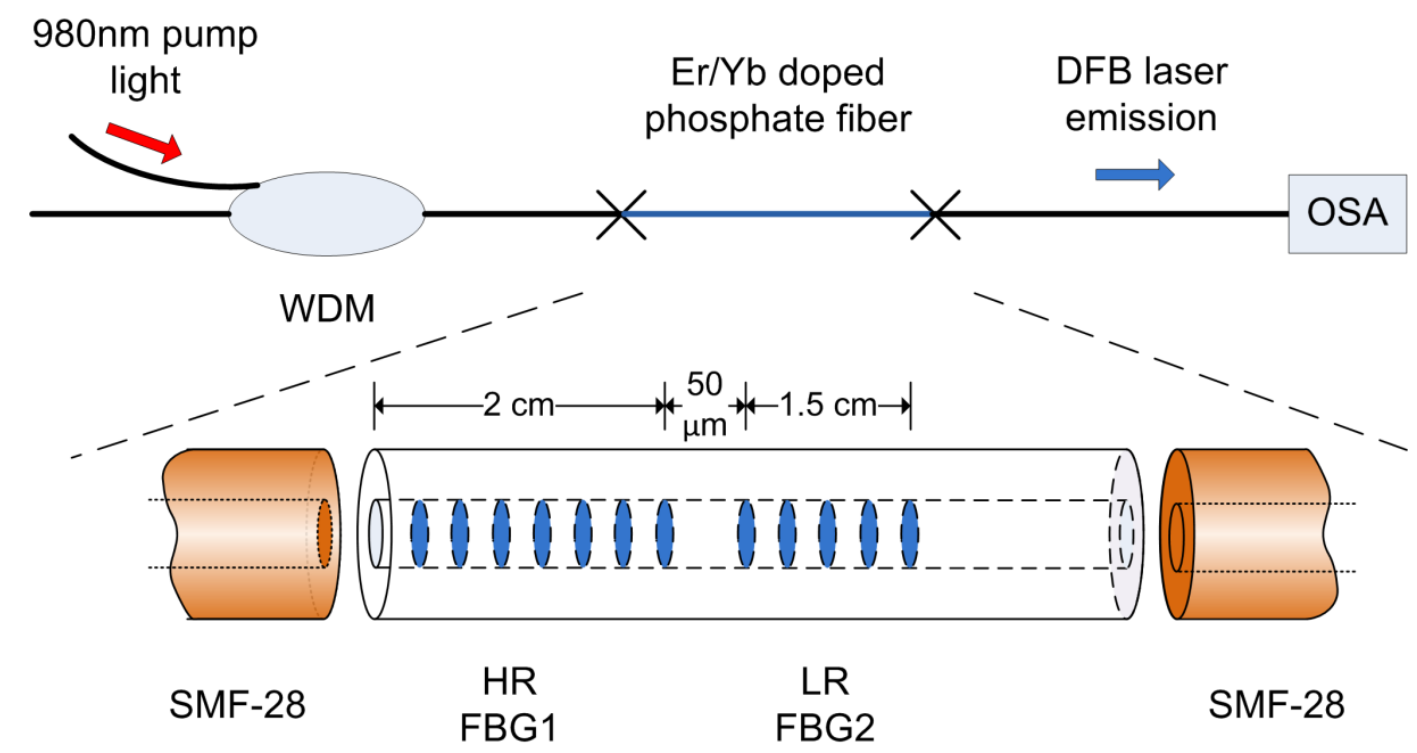

Figure 5.1 Schematic of the single-wavelength DFB phosphate fiber laser.

The experimental transmission spectrum of the DFB grating structure in the doped phosphate fiber along with the simulated spectrum is shown in Figure 5.2. In the measured spectra, the transmission dip of the DFB grating structure reaches $-16.85 \mathrm{~dB}$ at the peak wavelength of $1531.23 \mathrm{~nm}$. No clear resonant Fabry-Perot transmission peak is 
shown inside the grating bandwidth, because the linewidth of FP peak is beyond the optical spectrum analyzer (OSA) resolution of $0.01 \mathrm{~nm}$. Reasonable agreement is found between the measured and simulated transmission spectra of this DFB grating structure. As shown in the simulated spectra, the $50 \mu \mathrm{m}$ gap in the grating ensures that only one Fabry-Perot resonance exists within the grating bandwith. This will lead the DFB fiber laser to operate at robust single longitudinal mode without mode hopping.

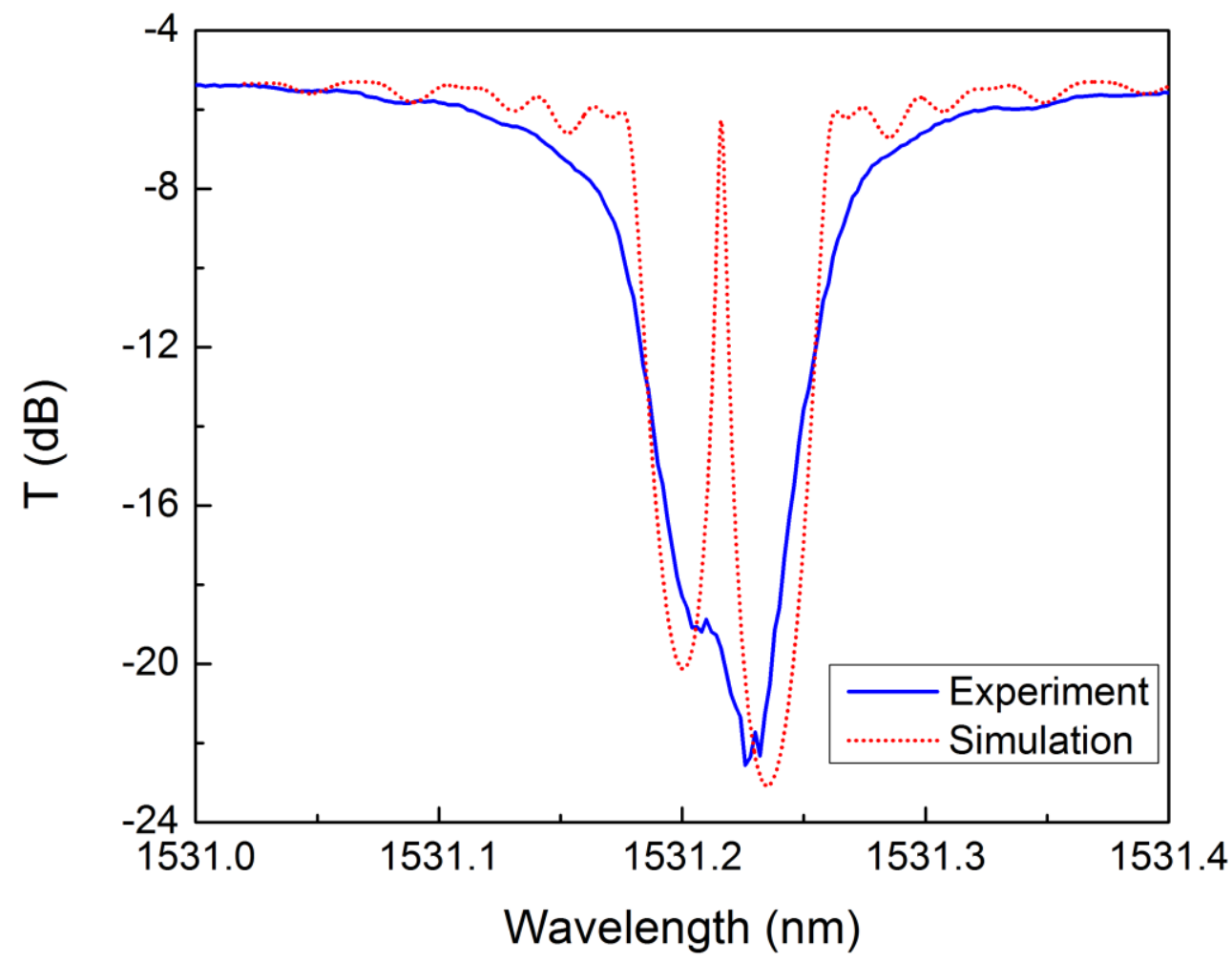

Figure 5.2 Experimentally measured (solid line) and simulated (dashed line) transmission spectra of the DFB grating in the $\mathrm{Er} / \mathrm{Yb}$ co-doped phosphate fiber.

Figure 5.3 shows the optical spectra of the single-wavelength DFB phosphate fiber laser pumped at launched pump powers of 132, 200 and $500 \mathrm{~mW}$ respectively. The laser is operating around threshold at a launched pump power of $132 \mathrm{~mW}$. As the launched pump power increases to $500 \mathrm{~mW}$, the laser power at the peak wavelength 
increases to $11.14 \mathrm{dBm}$. The signal-to-noise ratio of this single-wavelength fiber laser is higher than $70 \mathrm{~dB}$. It is worth noting that the lasing wavelength is almost stabilized at $1531.15 \mathrm{~nm}$ in spite of the big variation of pump power levels. This is helped by the fact that the laser cavity is passively cooled by mounting the active phosphate fiber on a metal plate. The excess heat generated by the high pump power is completely dissipated from the phosphate fiber to the metal plate.

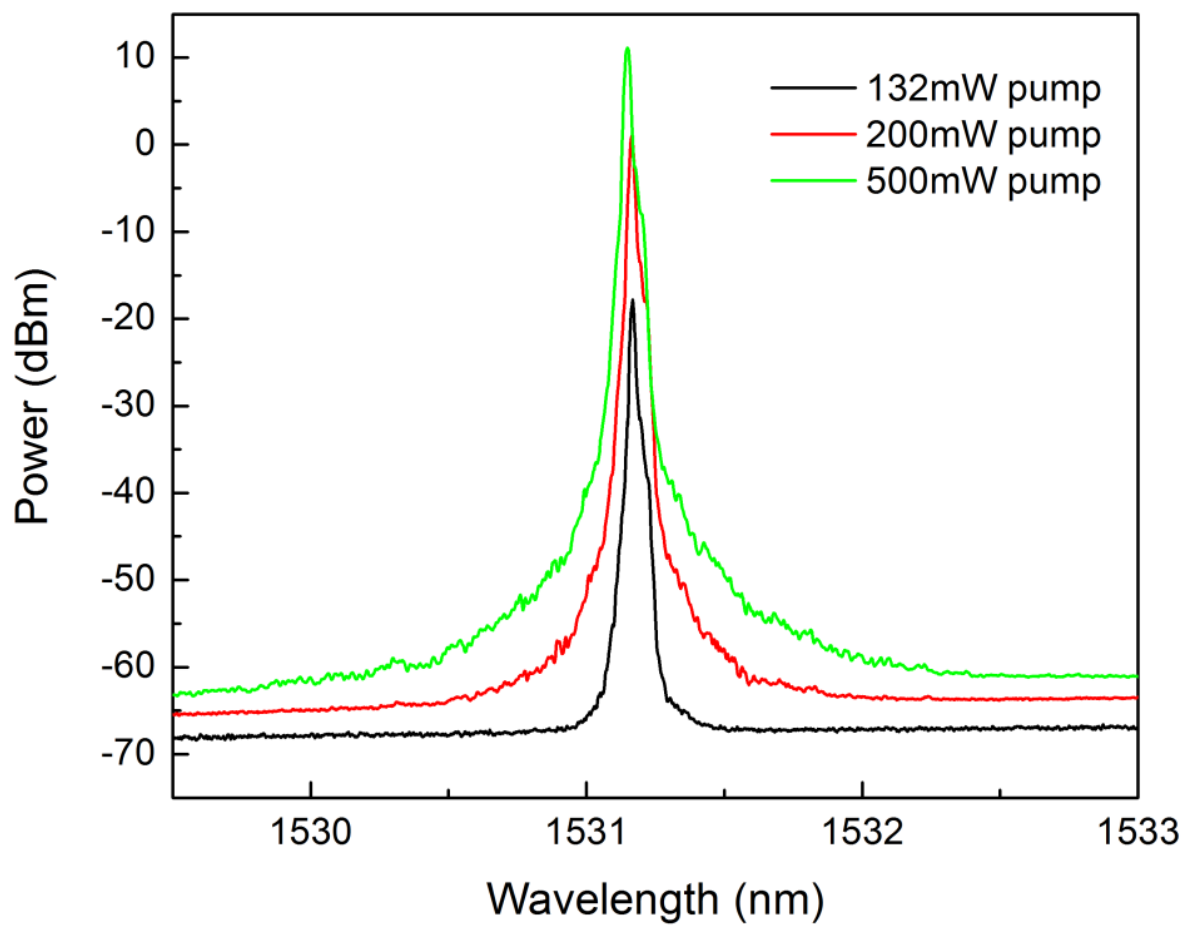

Figure 5.3 Optical spectra of the single-wavelength DFB phosphate fiber laser pumped at different launched pump power: 132, 200 and $500 \mathrm{~mW}$. 


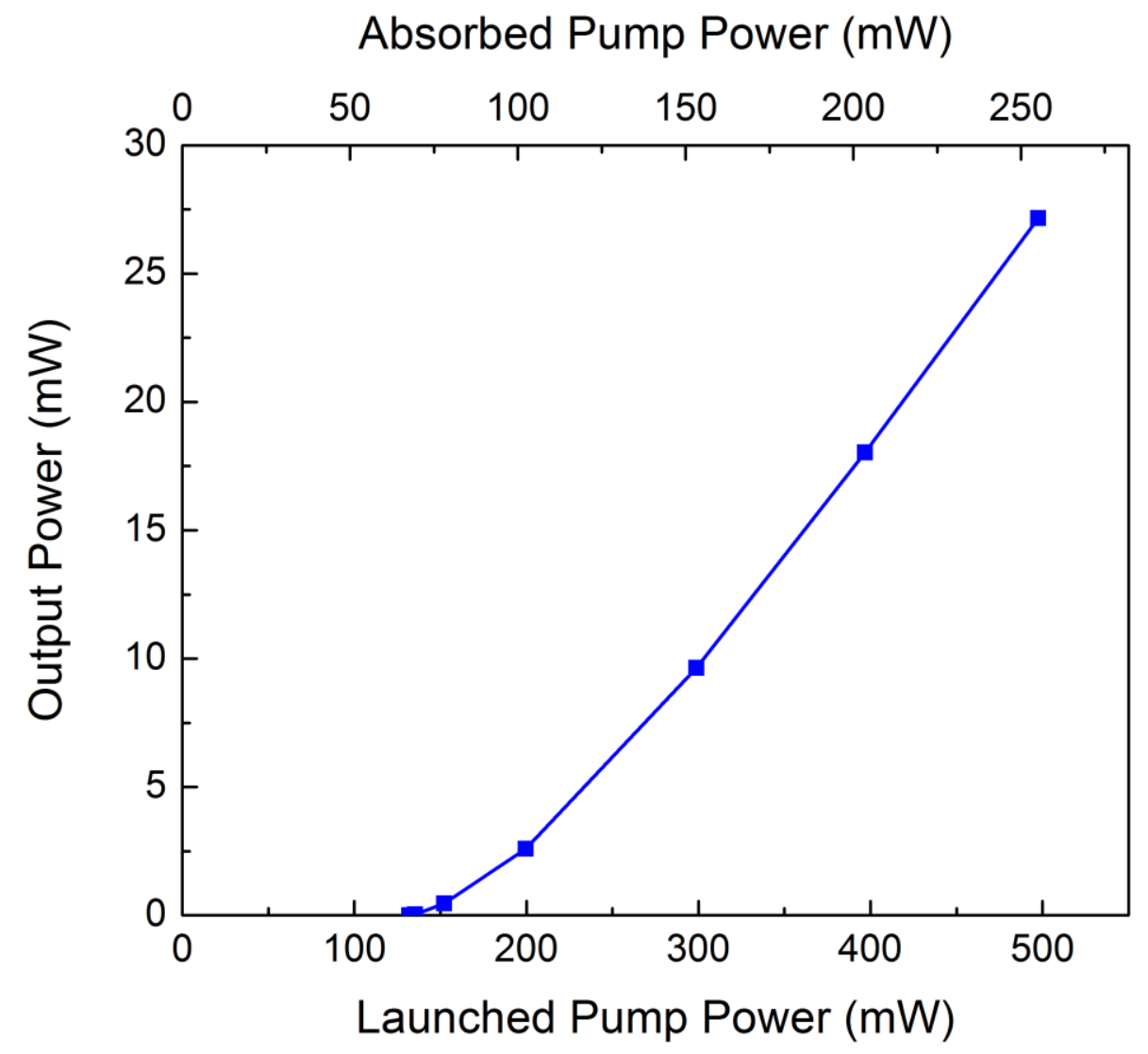

Figure 5.4 Output power as a function of launched pump power (bottom axis) and absorbed pump power (top axis) for the DFB fiber laser.

The laser output power plotted against the pump power for the single-wavelength DFB fiber laser is shown in Figure 5.4. The laser reaches threshold at a launched pump power around $132 \mathrm{~mW}$. The maximum laser output power is $27.5 \mathrm{~mW}$ as the launched pump power increases to $500 \mathrm{~mW}$. The slope efficiency with respect to launched power is $8.82 \%$ for this core-pumped DFB phosphate fiber laser. This slope efficiency is higher than that of the cladding-pumped DFB phosphate fiber laser [24,25]. Furthermore, if taking the splice loss of phosphate fiber chain and WDM coupling loss of the pump power into account, the slope efficiency with respect to the absorbed pump power is $17.2 \%$. 
The laser stability over time is checked by continually observing the optical spectra of the single-wavelength DFB fiber laser running for more than 45 min. Figure 5.5 shows the fluctuations of both the lasing wavelength and the peak power for the DFB fiber laser pumped at $500 \mathrm{~mW}$ for more than $45 \mathrm{~min}$. As shown in the figure, the laser power at the peak wavelength varies between 11.12 and $11.3 \mathrm{dBm}$ over a period of 1 hour. The fluctuation of the lasing wavelength of the DFB fiber laser is only $8 \mathrm{pm}$, which is even below the $0.01 \mathrm{~nm}$ resolution of the OSA. This observed fluctuation is mainly attributed to the measurement error of the OSA instead of the laser itself. The lasing wavelength should be more stable than the observed results.

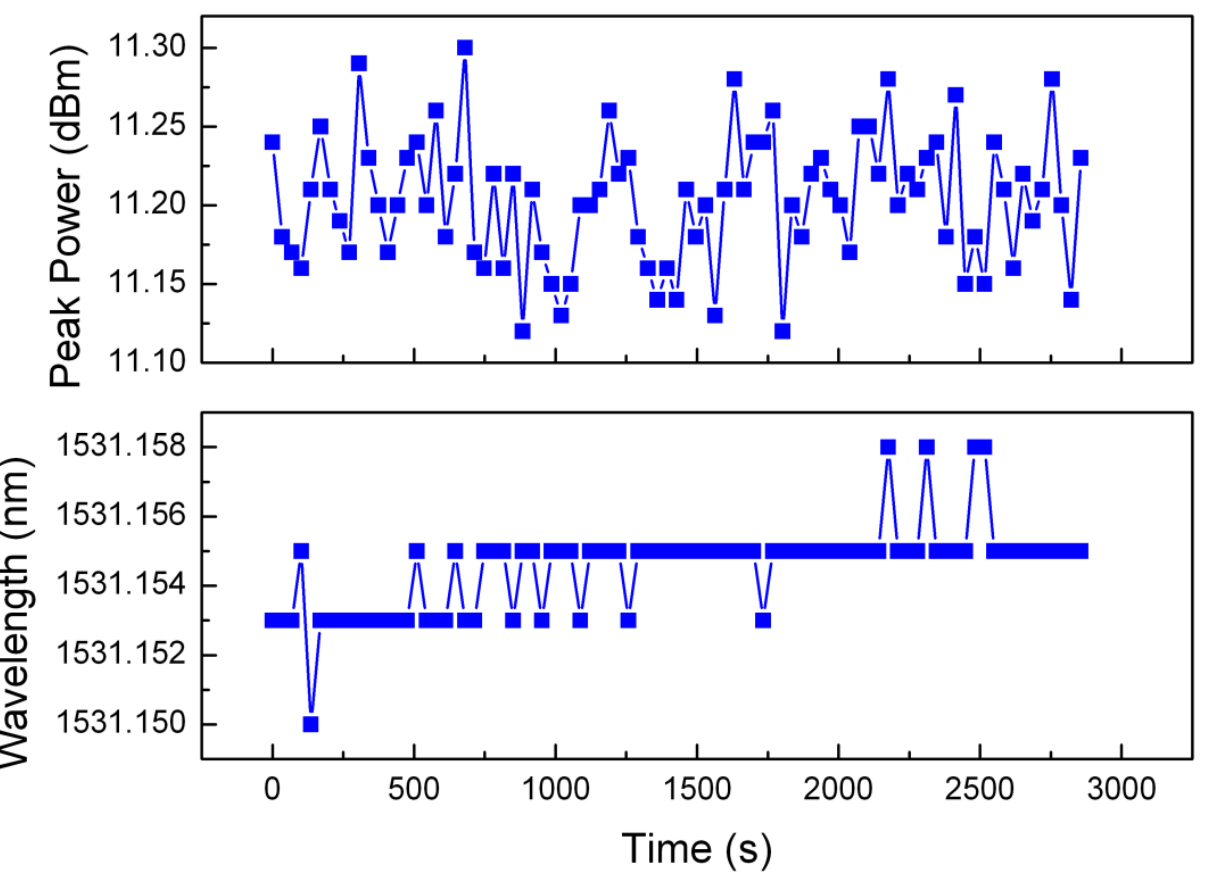

Figure 5.5 Fluctuations of both the lasing wavelength and peak power for the DFB fiber laser pumped at $500 \mathrm{~mW}$ for more than $45 \mathrm{~min}$.

The linewidth of the DFB fiber laser cannot be precisely measured by the OSA since it is well below the best resolution of the OSA. The heterodyne technique is used to 
characterize the narrow-linewidth DFB fiber laser. The laser signal is mixed with another narrow-linewidth laser (TUNICS T100S Tunable laser, linewidth $<150 \mathrm{kHz}$ ), and the mixed signal is then photodetected and measured by an electrical spectrum analyzer (ESA). Figure 5.6 shows the heterodyne spectrum of the single-wavelength DFB fiber laser pumped at $500 \mathrm{~mW}$. By adjusting the reference laser wavelength about $30 \mathrm{pm}$ longer than that of DFB laser signal under test, the beating frequency of the heterodyne signal is measured as $3.597 \mathrm{GHz}$, and the laser linewidth of the DFB fiber laser is about $20 \mathrm{MHz}$. The beating signal has a noticeable amplitude fluctuation and a $60 \mathrm{MHz}$ frequency drift, which is related to the output instability of the DFB fiber laser. It should be noted that there is another small beating signal at a frequency of $4.119 \mathrm{GHz}$ in the heterodyne spectrum. It indicates that the single-wavelength DFB fiber laser oscillates in dual polarization modes. The separation between two beating signals agrees with UVinduced birefringence as grating fabrication. Due to the small UV-induced birefringence on the active phosphate fiber during the grating fabrication, the laser will oscillate at the two orthogonal eigen polarization states. However, as a result of the difference between the cavity losses for each polarization, the laser still mainly emits at one polarization state. 


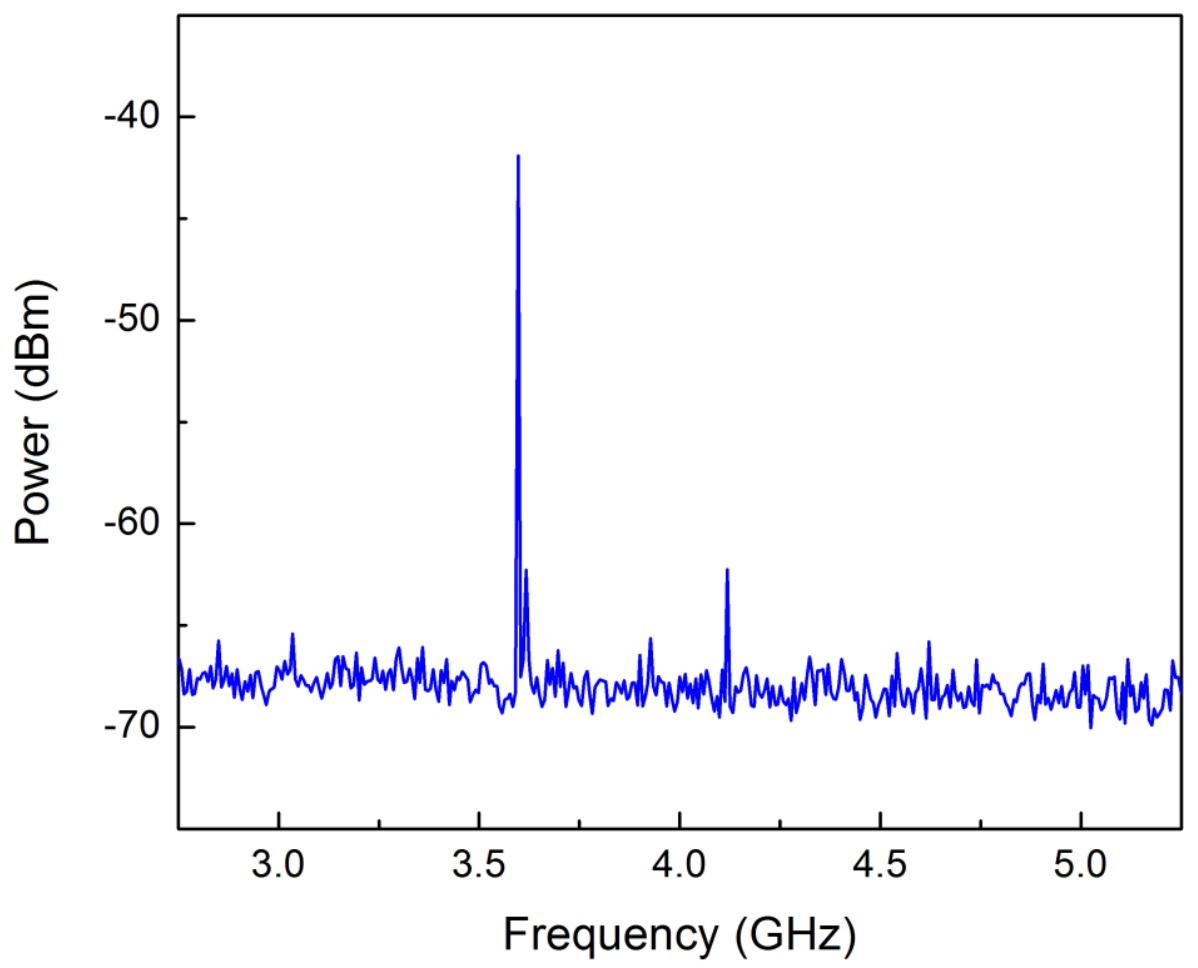

Figure 5.6 Heterodyne spectrum of the single-wavelength DFB fiber laser.

\subsection{Dependence of Laser Output Power on the DFB Grating Reflectivity}

Three DFB fiber lasers were fabricated to investigate the dependence of the laser output power on the DFB grating reflectivity. The transmission spectra of three DFB fiber laser cavities DFB1, DFB2 and DFB3 are plotted in Figure 5.7. The DFB grating structures DFB1, DFB2 and DFB3 were written with different UV irradiation times, and their grating reflectivities are in order of from low to high. The transmission notch depth of the grating structure DFB1, DFB2 and DFB3 are $7.36 \mathrm{~dB}, 9.47 \mathrm{~dB}$ and $16.55 \mathrm{~dB}$ respectively. It should be noted that the DFB3 laser cavity is the one reported in the previous section. 
All of three DFB fiber lasers are pumped up to $500 \mathrm{~mW}$ by a $980 \mathrm{~nm}$ laser diode, and single wavelength lasing emission is observed for all lasers by checking the optical spectra of laser signals. The output power as a function of launched pump power for the single-wavelength fiber lasers DFB1, DFB2 and DFB3 are plotted in Figure 5.8. As shown in the figure, the laser output powers of the three DFB fiber lasers are $7.1 \mathrm{~mW}$, $18.0 \mathrm{~mW}$ and $27.5 \mathrm{~mW}$ respectively, at the maximum launched pump power of $500 \mathrm{~mW}$. The laser output power and the slope efficiency monotonically increases as the DFB grating reflectivity increases from the level of DFB1 to that of DFB3.

There is a potential further improvement of the laser output power, which requires higher grating index modulation to form a DFB grating with higher reflectivity than DFB3. However, there should be an optimized value for the DFB grating reflectivity, over which the laser output power will drop down since the output coupling portion from the laser cavity is too low to couple the light out. In addition to UV photosensitivity of the active phosphate fiber, the fusion splice loss (about $1 \mathrm{~dB}$ per splice) between the phosphate fiber and the SMF-28 fiber is also a factor degrading the laser efficiency. This problem can be solved by optimizing splicing parameters used for phosphate-to-silica fiber splices. 


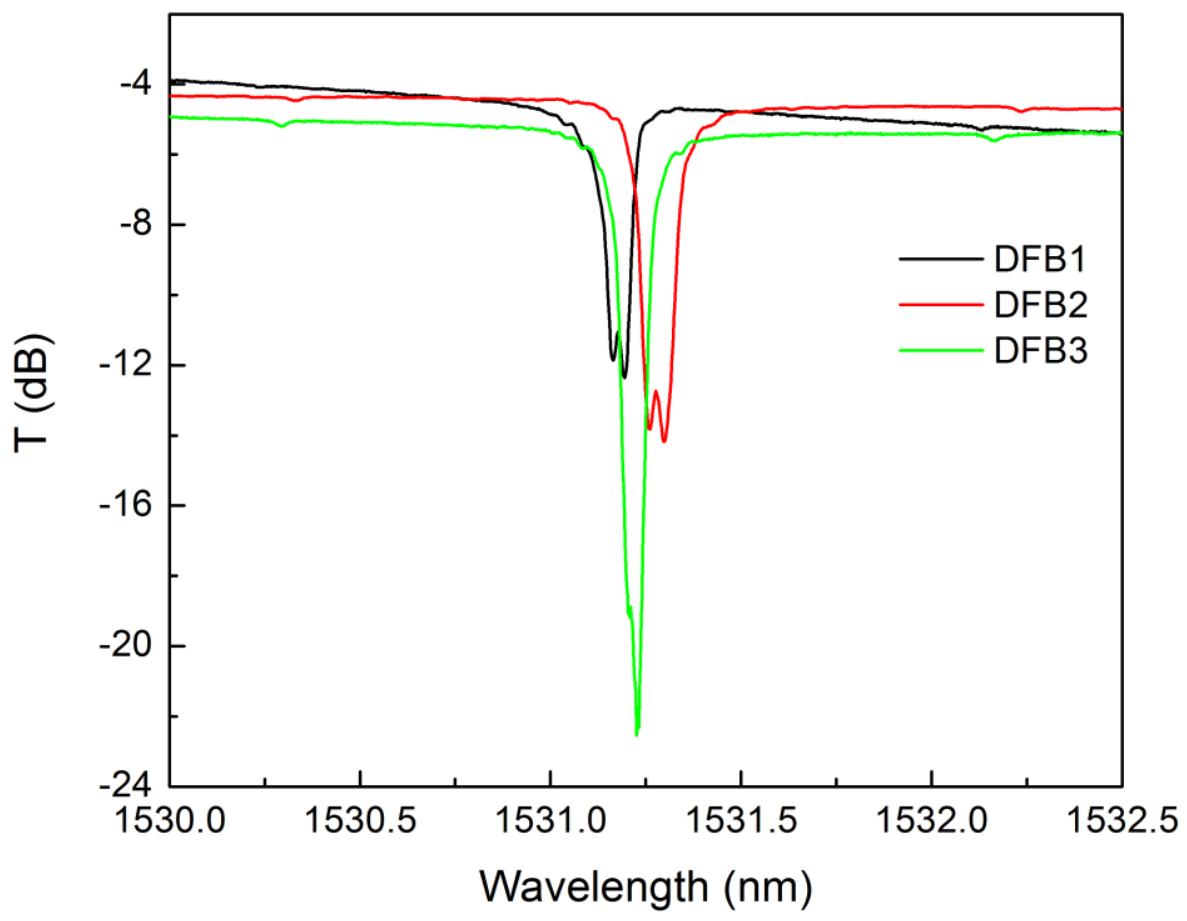

Figure 5.7 Transmission of the DFB gratings in the Er/Yb doped phosphate fiber: DFB1, DFB2 and DFB3.

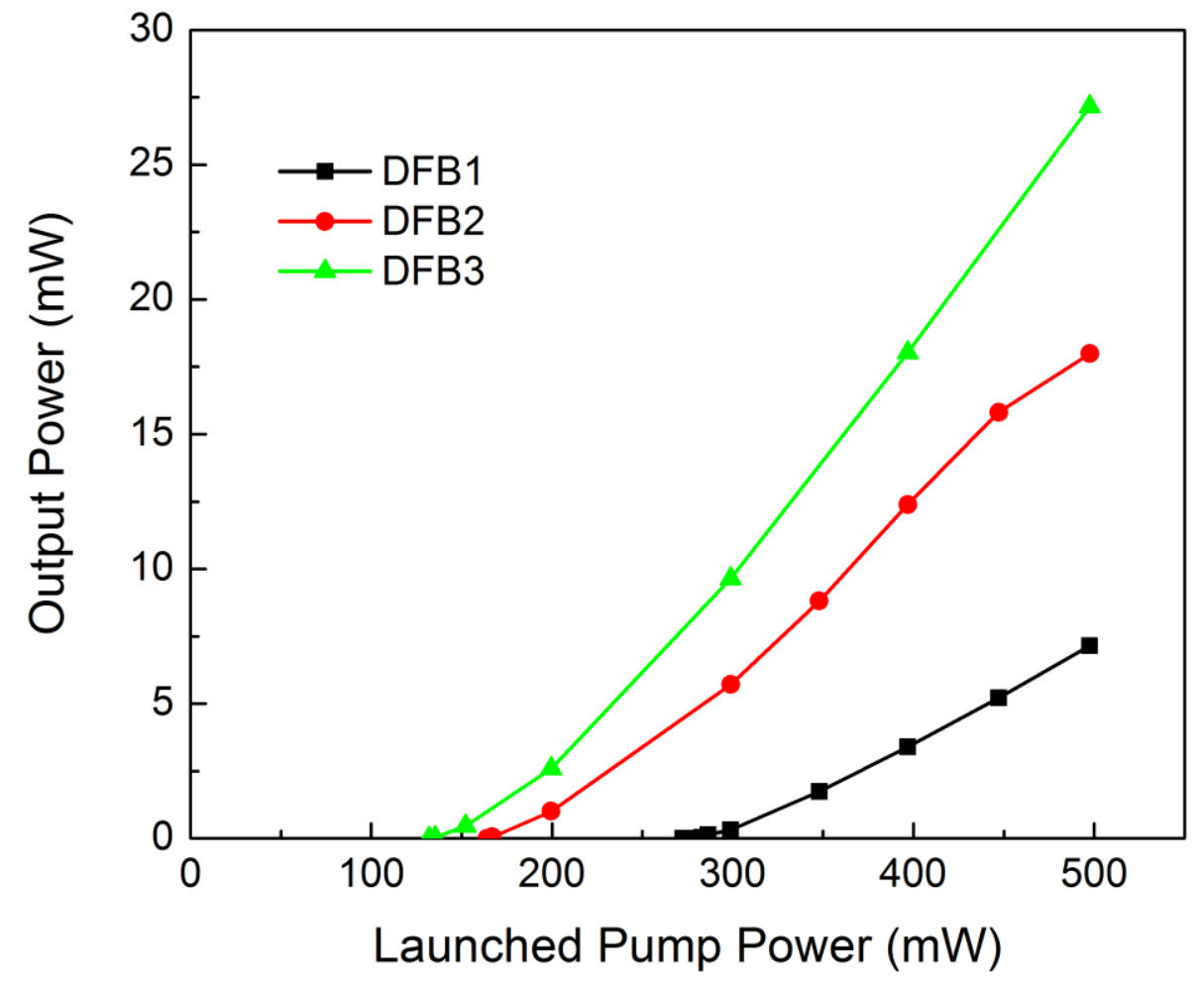

Figure 5.8 Output power as a function of launched pump power for the single-wavelength DFB fiber lasers: DFB1, DFB2 and DFB3. 


\subsection{Conclusion}

A 3.5-cm-long single-wavelength narrow-linewidth distributed feedback fiber laser is implemented in this chapter. The DFB grating structure is directly written into the 1\%-2\% Er/Yb doped phosphate glass fibers. A laser signal with an output power of 27.5 $\mathrm{mW}$ at a linewidth of $20 \mathrm{MHz}$ is achieved. The slope efficiency with respect to the absorbed pump power is $17.2 \%$. The dependence of slope efficiency on DFB grating reflectivity is also investigated. It shows that a further improvement of the slope efficiency will require a larger grating index modulation to achieve high-reflectivity laser cavity mirrors. 


\section{Chapter 6}

\section{Dual-wavelength Single-Longitudinal-Mode DBR}

\section{Phosphate Fiber Laser}

This chapter demonstrates the application of direct-writing FBGs with $193 \mathrm{~nm}$ irradiation in building a distributed Bragg reflector (DBR) fiber laser. A stable dual-wavelength single-longitudinal-mode laser emission is achieved in a 5-cm-long $\mathrm{Er} / \mathrm{Yb}$ doped phosphate fiber laser. The work in this chapter has been published in two papers $[81,82]$.

\subsection{Introduction}

Phosphate glass fiber is an important medium for building compact and monolithic optical fiber lasers, as the fiber is capable of hosting a higher concentration of Erbium and Ytterbium ions without causing cluster formation [83]. However, the lack of photosensitivity in phosphate glass fiber used to prevent the integration of UV-written intra-fiber gratings into the laser cavity. Many phosphate fiber lasers were built by incorporating external reflector mirrors, such as depositing thin film coatings on fiber facets or fusion splicing with conventional silica-based fiber Bragg gratings (FBGs) $[4,9-11,84,85]$. For these hybrid phosphate-silica glass devices, both optical losses and mechanical instabilities at the splicing points present inherent challenges due to the different thermal properties of the two glass types. Recently, our group has successfully written strong and thermally stable Bragg gratings directly in phosphate glass fibers using an $\operatorname{ArF}$ excimer laser $[12,60]$ and we have demonstrated compact and monolithic 
cladding-pumped distributed feedback (DFB) lasers in $\mathrm{Er} / \mathrm{Yb}$ co-doped phosphate fiber $[24,25,86]$. In reference [25], a spectrally narrow dual-wavelength laser emission is obtained by cascading two DFB phosphate fiber lasers with pump light propagating in the cladding of the laser chain. Dual-wavelength fiber lasers have attracted great interest because of their potential applications in optical communication, optical sensing, interferometry, and microwave photonic generation.

In this chapter, a dual-wavelength emission from one short monolithic corepumped distributed Bragg reflector (DBR) phosphate fiber laser is proposed and demonstrated. However, because erbium doped fiber is a homogenously broadened gain medium, the severe mode competition imposes a challenge to achieve simultaneous multiple wavelength oscillation. To establish stable dual-wavelength lasing at room temperature, various techniques have been employed to reduce the mode competition. These include exploiting the spatial hole burning effect in a linear cavity $[87,88]$, utilizing two spatially separated phase shifted DFB structures $[89,90]$, exploiting the polarization hole burning effect by using polarization-maintaining fiber Bragg gratings [91-94], inserting a piece of un-pumped Erbium-doped fiber as a saturable absorber $[95,96]$, and even incorporating a semiconductor optical amplifier as an inhomogenously broadened gain medium [97-100].

In this chapter, a 5-cm-long DBR cavity is formed by directly inscribing a pair of 2-cm-long uniform fiber Bragg gratings into Er/Yb co-doped phosphate fiber. We present detailed results and analysis about the DBR fiber laser fabrication and optical properties. This complete analysis helps to fully explain the underlying mechanism of the stable dual-wavelength lasing operation of the proposed fiber laser. The 1-cm-long separation 
between FBGs ensures that only two Fabry-Perot (FP) transmission resonances exist within the grating bandwidth. Therefore, only two longitudinal modes are oscillating in the laser cavity. A polarization analysis of the laser output shows that the two lasing modes belong to different linear polarization states owing to the UV-induced birefringence from the grating inscription. Hence, stable dual-wavelength emission with $38 \mathrm{pm}$ wavelength spacing is achieved by exploiting the spatial hole burning effect and the polarization hole burning effect to reduce mode competition. The characterization on the states of polarization of the dual-wavelength laser output, along microscopic images of the UV-induced surface damages on the phosphate glass fiber, suggests that the stable dual-wavelength lasing operation can be mainly attributed to the polarization hole burning effect. Compared to other dual-wavelength fiber lasers, the proposed DBR fiber laser has the advantages of simple structure, effective cost and ease of fabrication. Without using polarization maintaining fiber, the small UV-induced birefringence in the doped fiber is sufficient to achieve the stable dual-wavelength emission. The slope efficiency of the proposed dual-wavelength phosphate fiber laser is low. This is mainly because of the relatively low UV photosensitivity of phosphate fibers (compared to silica fibers) which prevents the achievement of high reflectivity gratings with optimized refractive index modulation along the fiber axis, as well as because of the large splice losses between the phosphate fiber and SMF-28 fiber. However, achieving a high slope efficiency is not the absolute requirement for most applications of this type of laser $[93,95,100]$. 


\subsection{Experimental Setup}

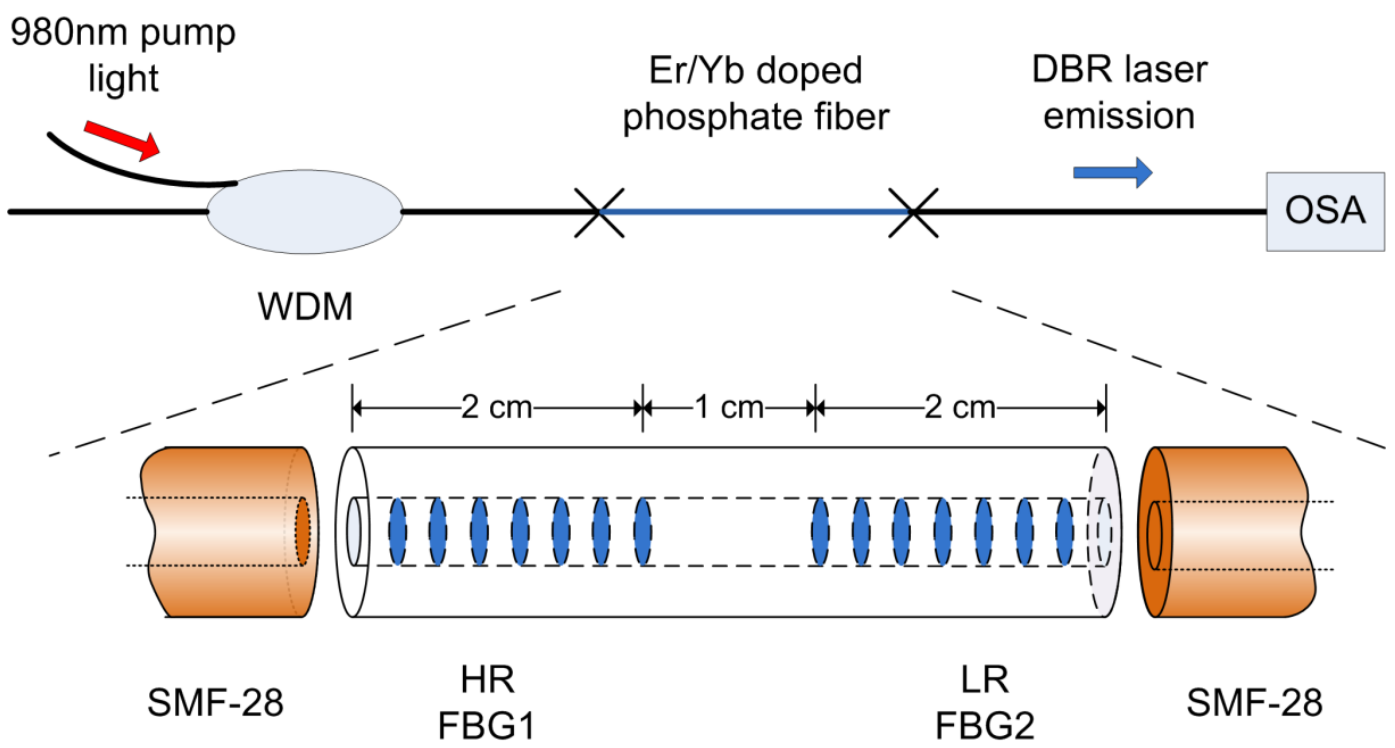

Figure 6.1 Schematic of the short dual-wavelength single-longitudinal mode DBR phosphate fiber laser.

The configuration of the proposed short dual-wavelength DBR phosphate fiber laser is shown in Figure 6.1. A $980 \mathrm{~nm}$ laser diode delivers up to $500 \mathrm{~mW}$ pump light to the doped phosphate fiber through a 980/1550 nm wavelength division multiplexer (WDM). We measure the laser signal in the same direction as the pump light propagation in this codirectional-pump configuration. The whole DBR laser cavity is fabricated on a 5-cm-long Er/Yb co-doped phosphate fiber, whose core is doped with $1 \mathrm{wt} \% \mathrm{Er}_{2} \mathrm{O}_{3}$ and 2 $\mathrm{wt} \% \mathrm{Yb}_{2} \mathrm{O}_{3}$. The phosphate fiber was fusion spliced to standard telecommunications fiber pigtails (Corning SMF-28) for compatible use with other devices. Intracore Bragg gratings were directly written in the phosphate glass fiber using a phase mask irradiated by intense UV radiation from a pulsed $193 \mathrm{~nm}$ ArF excimer laser. As shown in Figure 6.1, two uniform FBGs (FBG1 and FBG2), each $2 \mathrm{~cm}$ long, were inscribed on each side of the phosphate fiber individually. The unexposed separation between them is $1 \mathrm{~cm}$ 
long. During grating fabrication, FBG1 was first written with a 2-cm-long phase mask, and then FBG2 was written with the same phase mask after the fiber had been manually translated to its desired position. To keep the resonance peaks of the gratings matched, the same tension was applied on the phosphate fiber during the fabrication of both gratings. The reflectivities of FBG1 and FBG2 are $95 \%$ and $90 \%$, and the lower reflectivity FBG2 is used as the output coupler of the laser.

\subsection{Experimental Results and Discussion}

The spectral characteristics of the DBR gratings were measured with an optical spectrum analyzer (OSA). Figure 6.2 illustrates the transmission spectra of a single grating FBG1 and of the dual-grating DBR structure written in the doped phosphate fiber. The Bragg resonances of the gratings FBG1 and FBG2 are at $1531.25 \mathrm{~nm}$. The FP transmission maxima are not clearly observed in the grating rejection band, mainly because the narrow bandwidths of FP transmission peaks are beyond the resolution limit $(0.01 \mathrm{~nm})$ of the OSA and also because of losses within the FP cavity when the fiber is not pumped [101]. For this FP cavity the theoretical wavelength spacing between the transmission resonance peaks is calculated to be $38 \mathrm{pm}$ according to the formula given in [102]. Therefore, only two FP resonances can oscillate within the about $100 \mathrm{pm} 3 \mathrm{~dB}$ bandwidth of the FBGs. 


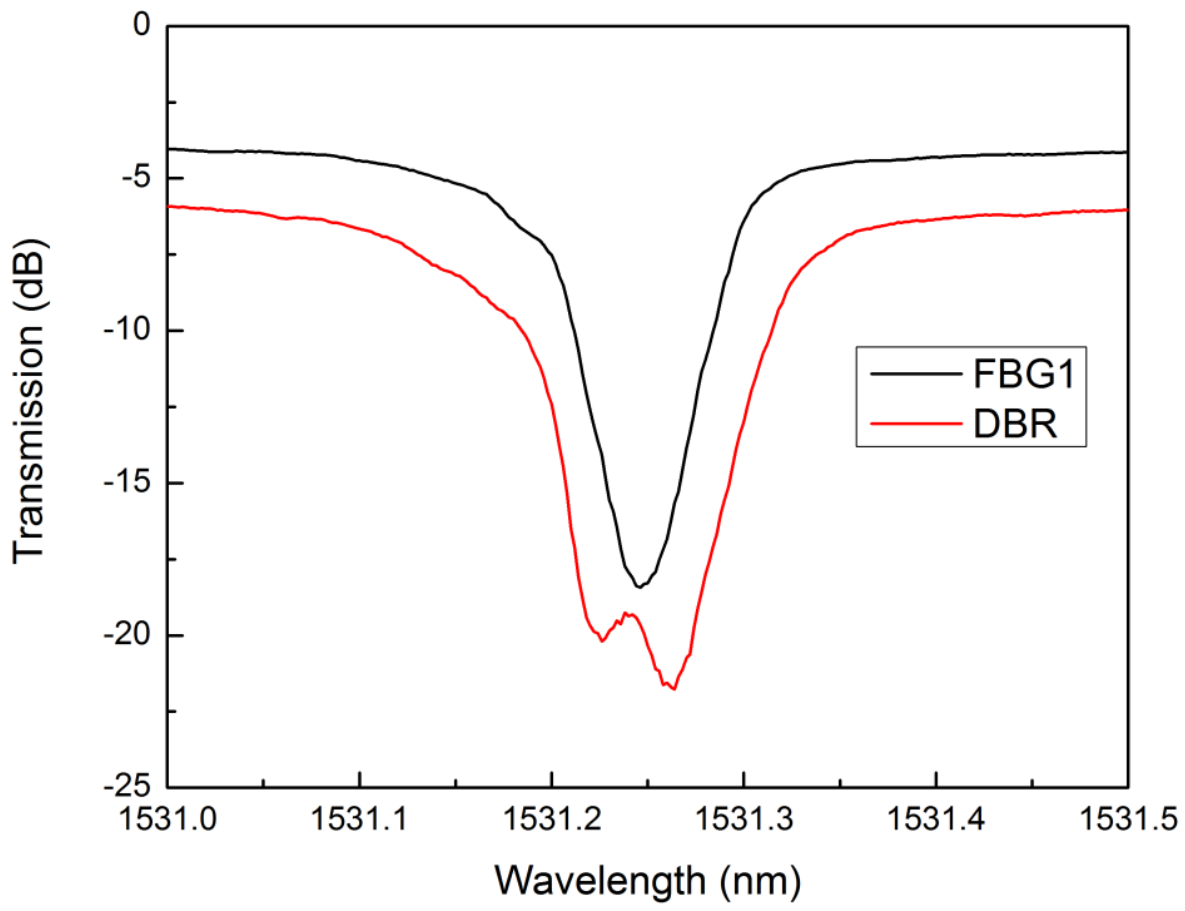

Figure 6.2 Transmission spectra of the uniform FBG1 and the DBR grating structure in the $\mathrm{Er} / \mathrm{Yb}$ doped phpshate fiber.

The fabricated DBR cavity is then employed to build the monolithic dualwavelength phosphate fiber laser, according to the schematic previously shown in Figure 6.1. As the heavily doped fiber generates the excessive heat upon high pump power, in this forward pump configuration FBG1 will experience a larger heat-induced red-shift in wavelength than FBG2. This wavelength mismatch poses a potential difficulty in achieving laser oscillation. To avoid the heat-induced wavelength mismatch between FBG1 and FBG2, a passive cooling scheme is used by mounting the doped fiber on a metal plate.

Figure 6.3 illustrates the optical spectra of the DBR phosphate fiber laser at launched pump powers of 350 and $500 \mathrm{~mW}$, respectively. It should be noted that the real launched pump powers into the doped phosphate fiber are only 223 and $319 \mathrm{~mW}$ because of the large total splice losses between $980 \mathrm{~nm}$ laser diode pigtail and the doped 
phosphate fiber. Dual-wavelength lasing at the designed Bragg wavelength is observed, and the optical signal to noise ratio is about $60 \mathrm{~dB}$. The linewidths of each lasing peak are too narrow to be resolved by the OSA. The wavelength spacing of the two lasing peaks is $38 \mathrm{pm}$, which agrees with the theoretical longitudinal mode spacing of this DBR structure. The output powers at the two laser emission peaks are nearly equal at a pump power of $350 \mathrm{~mW}$, but when the pump power is increased to $500 \mathrm{~mW}$, the long wavelength lasing peak is favored. This suggests that the cavity loss of the two longitudinal lasing modes is pump power dependent. We note that, although the laser cavity is passively cooled by mounting the fiber on a metal plate, during laser operation the transmission spectra of our DBR structure changes slightly as a result of pump induced residual temperature variations.

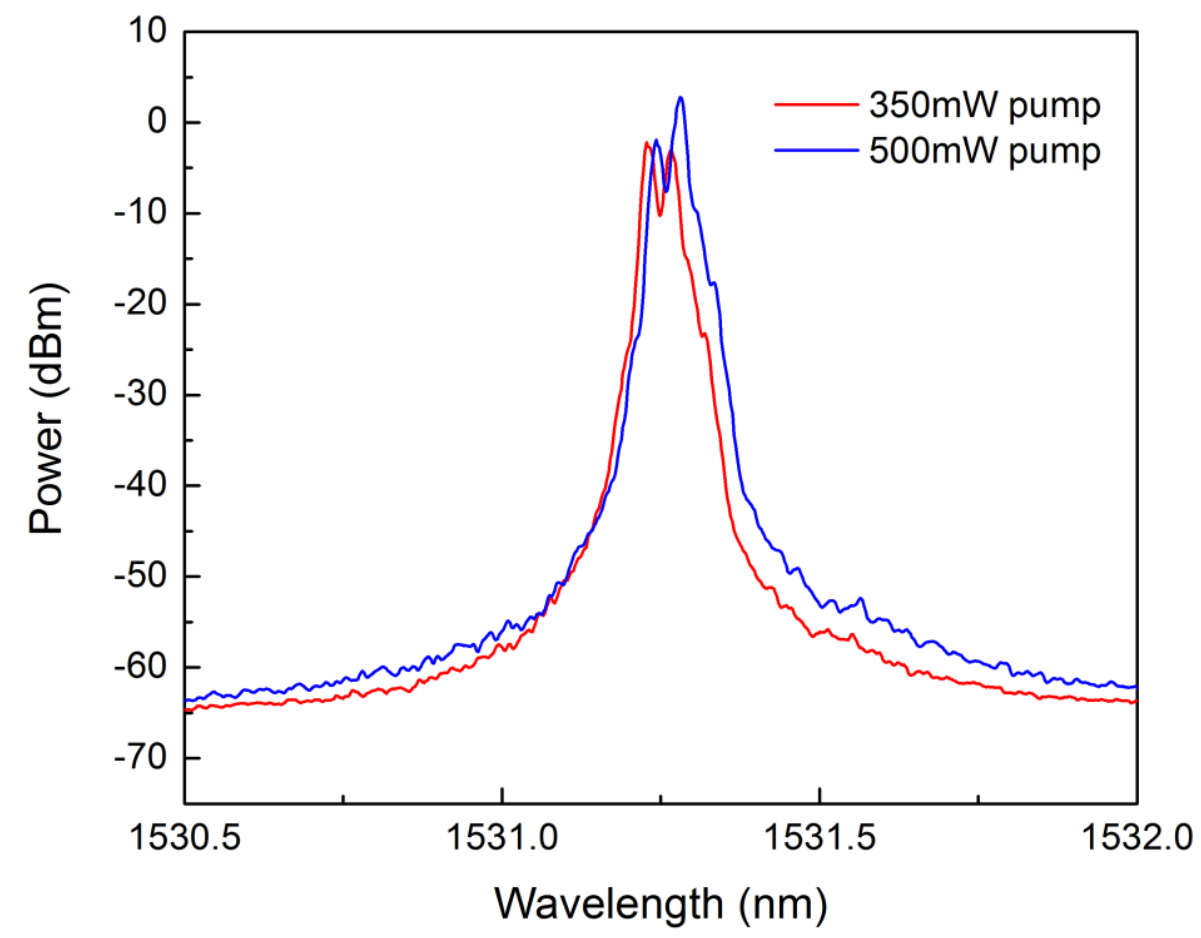

Figure 6.3 Optical spectra of the dual-wavelength DBR phosphate fiber laser pumped at $350 \mathrm{~mW}$ and $500 \mathrm{~mW}$. 
In order to investigate long term stability of the laser output, the optical spectra of the dual-wavelength fiber laser pumped at $500 \mathrm{~mW}$ are measured at time intervals of 1 min, as shown in Figure 6.4(a). The laser spectrum is stable over the observation period. The stabilities of output power and lasing wavelength of the two lasing peaks are plotted in Figure 6.4 (b) and Figure 6.4 (c).

As shown in the figures, both lasing wavelengths are very stable with variations of less than $10 \mathrm{pm}$, which is the resolution limit of the OSA. The peak output powers at each lasing wavelength exhibit different fluctuations: the strong lasing peak with an output power of $2.98 \mathrm{dBm}(1.99 \mathrm{~mW})$ varies by $0.48 \mathrm{~dB}$ over the observation period. In contrast, a larger relative power variation of up to $1.53 \mathrm{~dB}$ is observed for the weak lasing peak with an output power of $-1.16 \mathrm{dBm}(0.77 \mathrm{~mW})$. In absolute terms, the peak output powers fluctuate at both emission wavelengths by $+/-0.1 \mathrm{~mW}$. The laser performance is very stable over long-term period. One additional measurement was taken after 1 hour of the laser continuously running, showing consistent wavelength and output power with those measured during the first 15 minutes. It appears difficult at this time to increase the slope efficiency of this structure beyond about $1 \%$, as achieved here, because of the combination of constraints that come into play simultaneously: basically the limited maximum index modulation of this photosensitive process makes it difficult to achieve laser cavity mirrors with high reflectivity and sufficient bandwidth to cover the two lasing wavelengths. The index modulations of FBG1 and FBG2 are $7.1 \times 10^{-5}$ and $5.8 \times 10^{-5}$ respectively. A further improvement of index modulation can be accomplished by increasing the pulse fluence of the UV laser used for grating fabrication, or employing a 

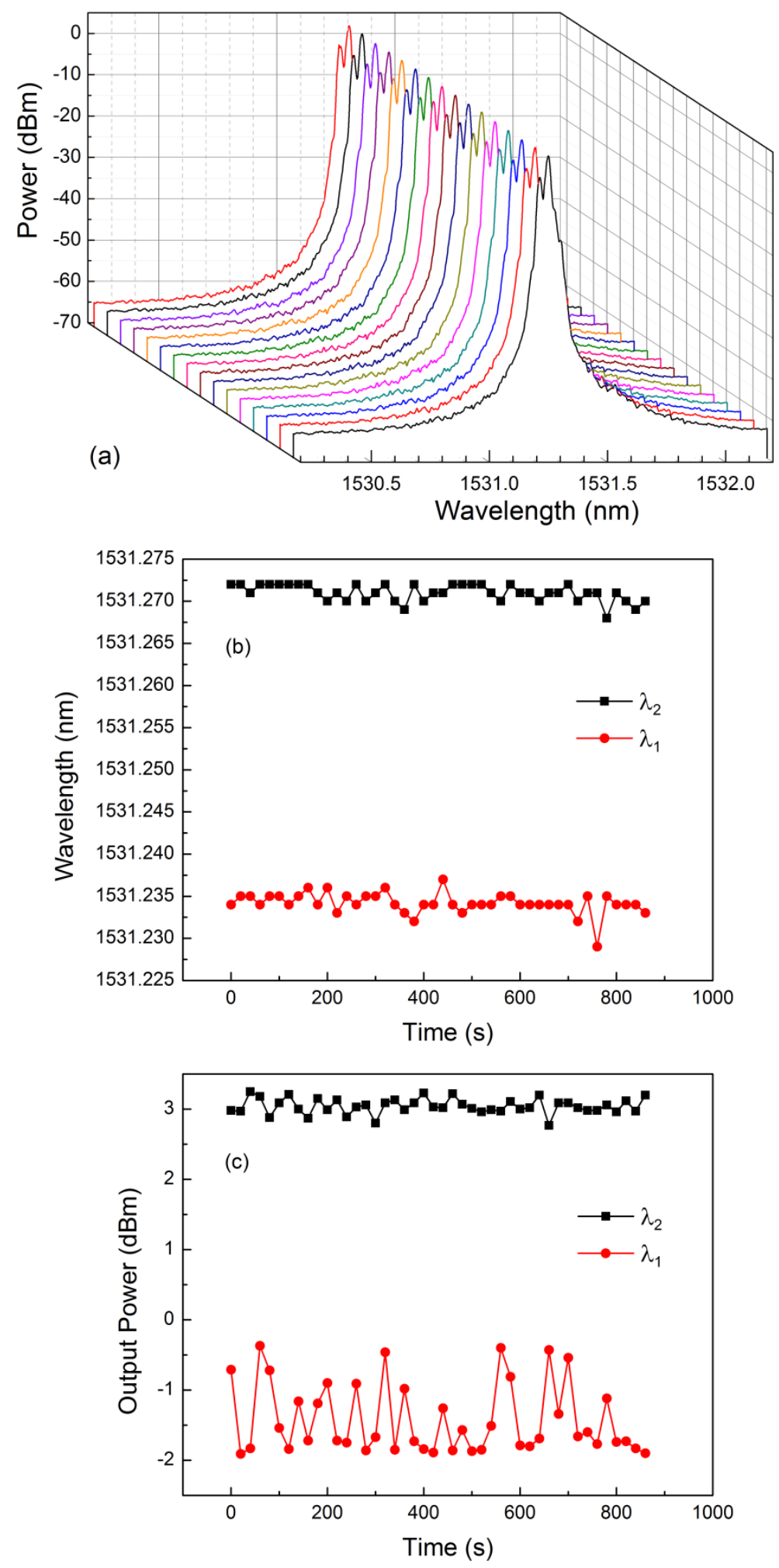

Figure 6.4 (a) Laser output spectra for 16 continuous sweeps taken at 1 min interval; fluctuation of (b) wavelengths and (c) peak output powers for dual- wavelength laser pumped at $500 \mathrm{~mW}$. 
thermal growth process by heating gratings at high temperatures which is a unique feature for phosphate FBGs $[12,60]$. Fortunately, high slope efficiency is not an absolute requirement for this type of laser application.

Figure 6.5 shows the measured microwave signal generated by photo detecting the dual-wavelength DBR fiber laser. The measurement is performed with an electrical spectrum analyzer (ESA) operating at a resolution bandwidth of $3 \mathrm{MHz}$ and a sweep span of $600 \mathrm{MHz}$. The beating signal has noticeable amplitude fluctuations due to the gain competition of the two lasing peaks, but its frequency is stabilized at $4.58 \mathrm{GHz}$ with a drift of less than $30 \mathrm{MHz}$. The 3-dB bandwidth of the signal is approximately $4 \mathrm{MHz}$. No strong microwave signal is found at other frequencies, meaning that both lasing peaks correspond to a single longitudinal mode.

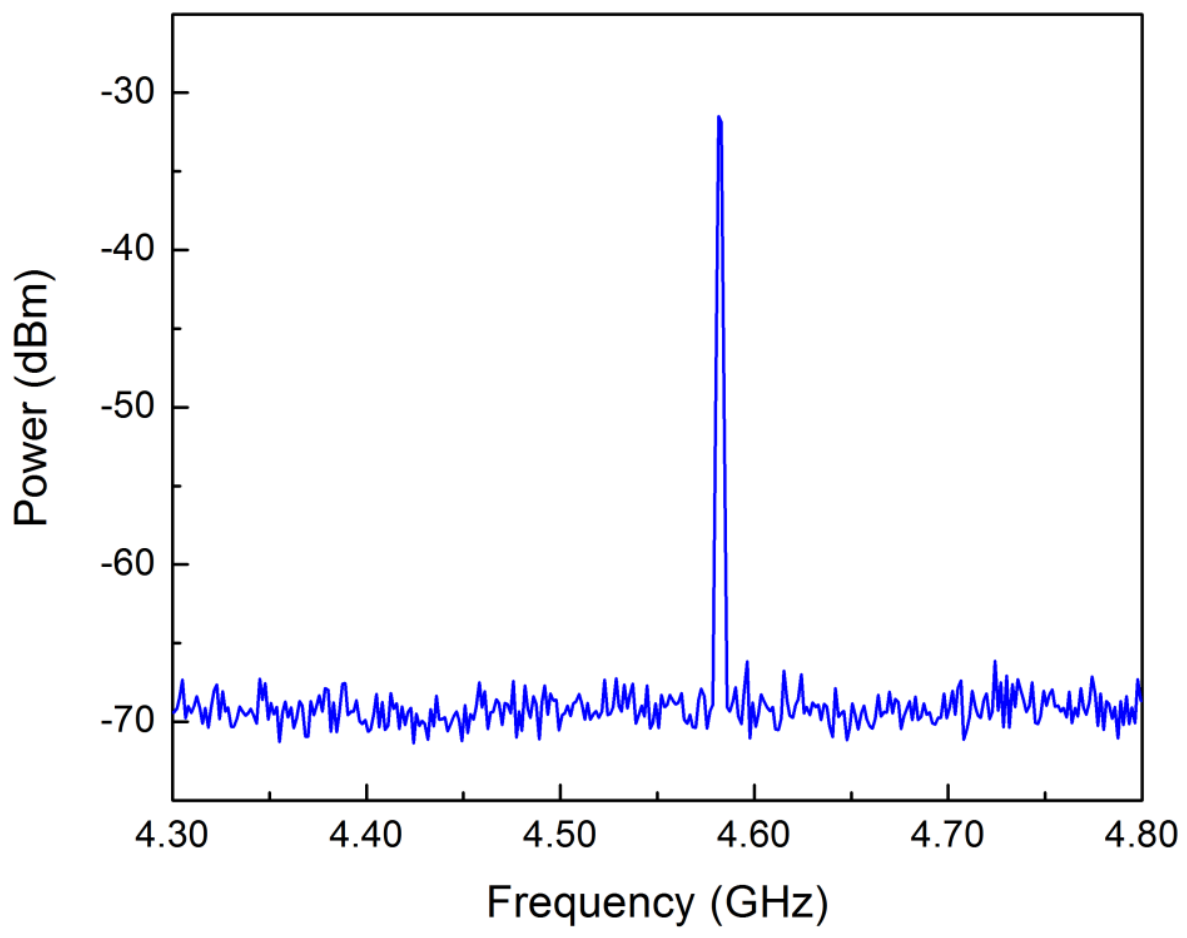

Figure 6.5 Electrical spectrum of the microwave signal generated by the dualwavelength DBR phosphate fiber laser. 
The polarization state of the dual-wavelength laser is characterized with an analyzer and the OSA. The laser output spectra through the analyzer oriented at different angles are shown Figure 6.6. The short and long lasing wavelengths are assigned as $\lambda_{1}$ and $\lambda_{2}$. The reference angle $\left(0^{\circ}\right)$ is defined for the angle corresponding to the maximum power for the dominant longitudinal lasing mode (at $\left.\lambda_{2}\right)$. Rotation of the analyzer by $90^{\circ}$ minimizes the power of the long wavelength lasing line but the maximum power output of the short wavelength $\left(\lambda_{1}\right)$ lasing line occurs at an analyzer angle of $70^{\circ}$. The polarization extinction ratio (PER) is about $20 \mathrm{~dB}$ at $\lambda_{1}$ and $10 \mathrm{~dB}$ at $\lambda_{2}$. The polarization states of $\lambda_{1}$ and $\lambda_{2}$ are schematically plotted in the inset of Figure 6.6.

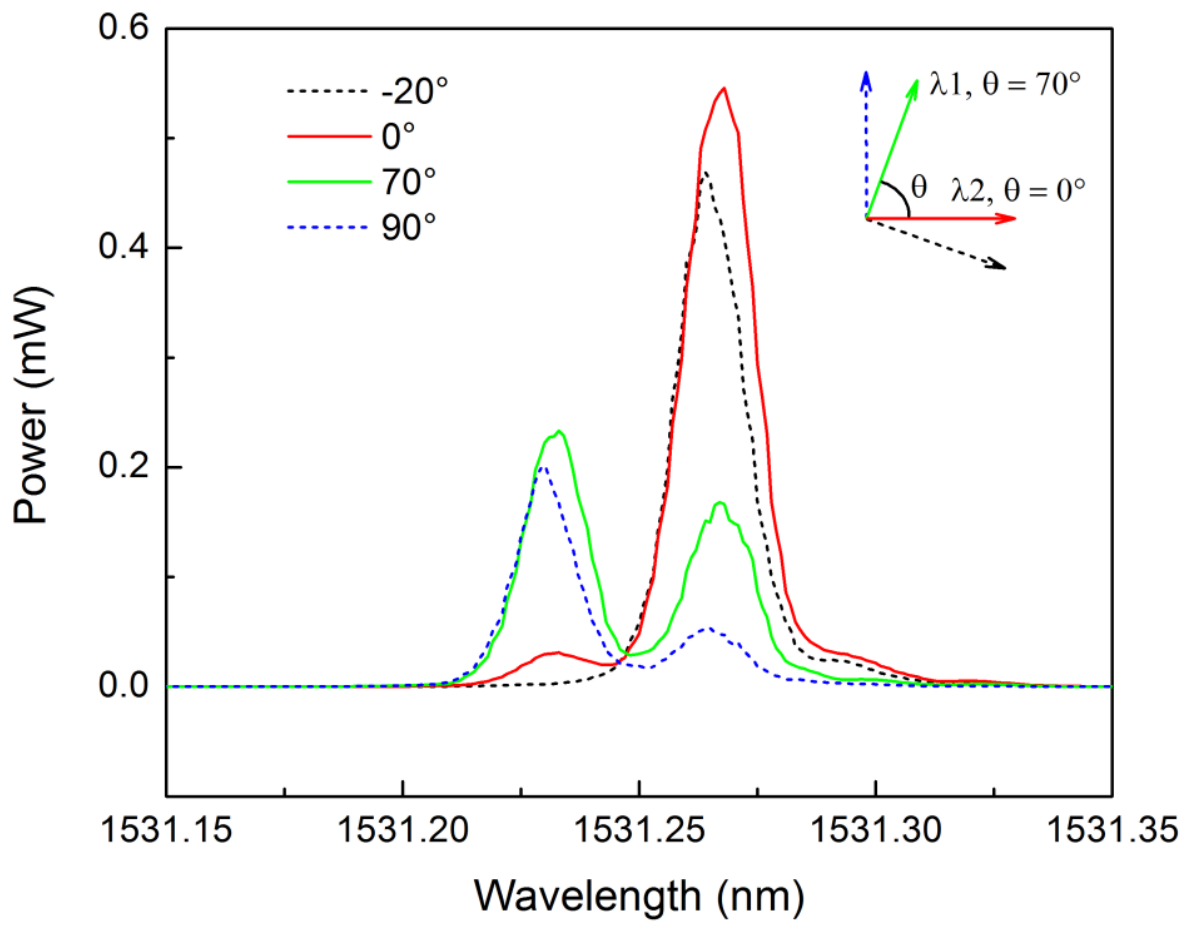

Figure 6.6 Laser output spectra transmitted through a polarization analyzer at different angles. The inset shows the polarization states of two lasing lines $\left(\lambda_{1}\right.$ and $\lambda_{2}$ : short and long lasing wavelengths). 
Ideally, the polarization hole burning effect is most effective for orthogonal polarization states but this does not appear to be the case here. This deviation of the angle between polarization states has been determined to originate from the grating fabrication process. As the FBGs are side-written with UV exposure from one direction, a UVinduced birefringence is introduced for each FBG. Because of the polarization hole burning effect, the UV-induced birefringence favors light to lase along the orthogonal eigen polarization states for FBGs. The origin of the $70^{\circ}$ rotation between the two lasing polarizations was verified to come from an accidental rotation of fiber by the same angle during the manual translation of the fiber between the fabrication of FBG1 and FBG2. This is confirmed by the following observation.

Figure 6.7 shows microscope images of the cladding surface on the ends of the UV-induced FBGs. As the phosphate glass is softer than silica glass, an ablation effect readily occurs under intense UV exposure. During the UV inscription of the gratings, small and shallow surface damage (as shown in Figure 6.7 (c)) is caused on the phosphate fiber outer cladding surface. Carefully rotating the fiber under microscope objective lens, and comparing the orientation angles of the damage for FBG1 and FBG2, the rotation angle between the UV incident directions is found to be about $70^{\circ}$. The above observation confirms that the stable dual-wavelength oscillation in the DBR cavity is mainly owing to a polarization hole burning effect. 


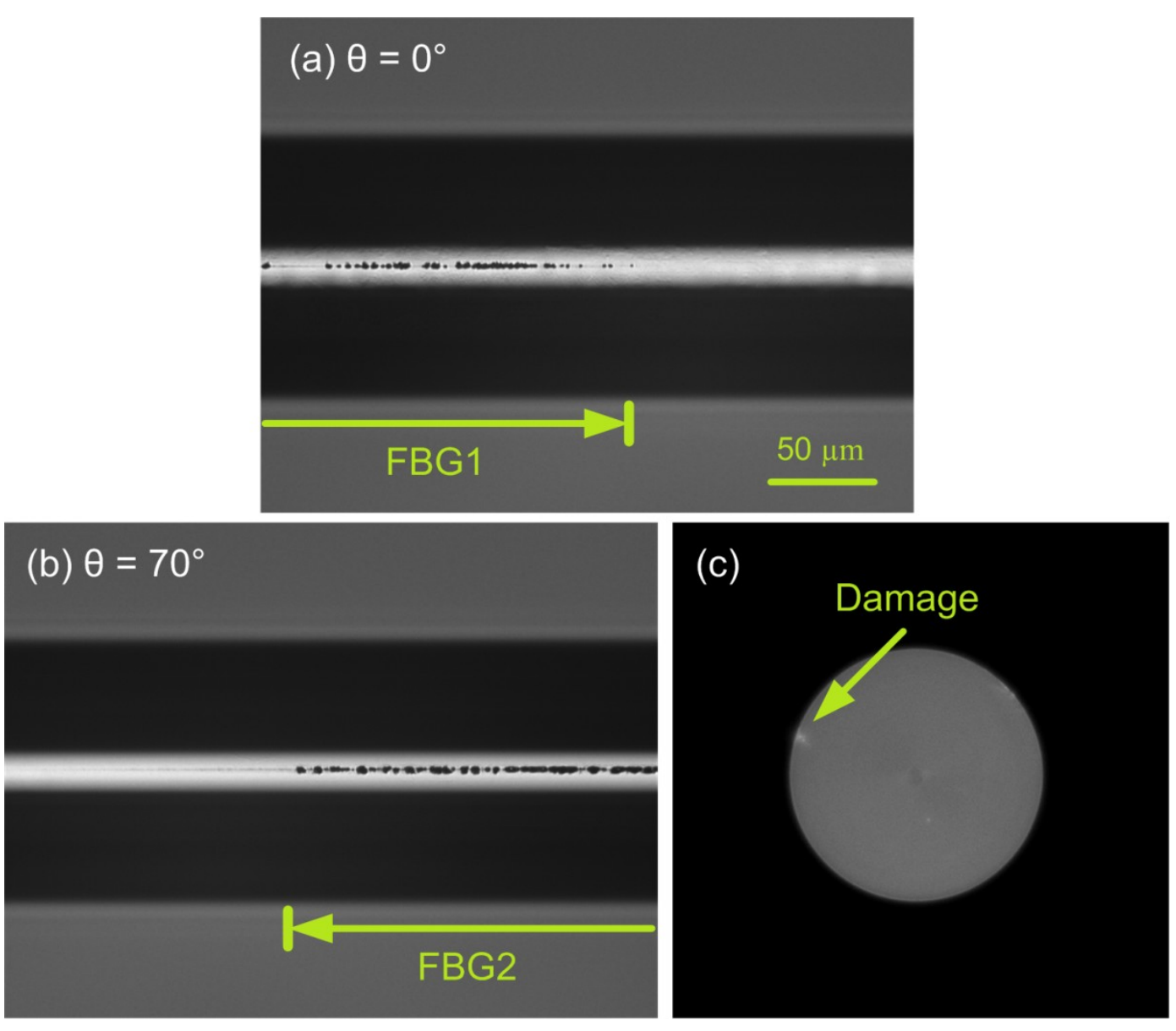

Figure 6.7 Microscope images of UV-induced damages on the phosphate fiber cladding surface for (a) FBG1 with rotation angle $\theta=0^{\circ}$; (b) FBG2 with rotation angle $\theta=70^{\circ}$; (c) cross-section of a cleaved FBG.

\subsection{Conclusion}

A 5-cm-long core-pumped monolithic DBR fiber laser is fabricated by directly writing Bragg gratings in highly Er/Yb co-doped phosphate fiber. Stable narrow-linewidth dualwavelength emission with a wavelength spacing of $38 \mathrm{pm}$ and a total emitted power of $2.8 \mathrm{~mW}$ is obtained from this DBR fiber laser. A microwave signal at a frequency of 4.58 $\mathrm{GHz}$ and stability of $30 \mathrm{MHz}$ is generated by this dual-wavelength fiber laser. 


\section{Chapter 7}

\section{Conclusions and Future Work}

\subsection{Conclusions}

The initial motivation of this thesis is to address the challenge of direct-writing fiber Bragg gratings into phosphate glass fibers. Strong gratings have been inscribed into $\mathrm{Er} / \mathrm{Yb}$ doped and undoped fibers using $193 \mathrm{~nm} \mathrm{ArF} \mathrm{excimer} \mathrm{laser} \mathrm{and} \mathrm{standard} \mathrm{phase}$ mask technology. An undesirable side effect of FBGs fabrication is discovered that a photodarkening loss of about $1 \mathrm{~dB} / \mathrm{cm}$ at C-band is also induced by UV irradiation. This brings our research effort to fully characterize the photodarkening effect by measuring its broadband VIS-NIR absorption spectrum. Photo-bleaching and thermal-bleaching processes have been demonstrated to be effective in fully erasing PD loss in phosphate fibers. Finally, the application of strong direct-written Bragg gratings in $\mathrm{Er} / \mathrm{Yb}$ doped phosphate fiber has been demonstrated in a short fiber laser. Both short monolithic single-wavelength DFB and dual-wavelength DBR phosphate fiber lasers have been successfully realized.

A complete investigation of photosensitivity of $\mathrm{Er} / \mathrm{Yb}$ doped and undoped

phosphate glass fibers has been carried out in Chapter 3, using irradiation of intense 193 $\mathrm{nm}$ ArF excimer laser light through a phase mask. Er/Yb doping is found to reduce the photosensitivity of phosphate fibers, and leads to UV-induced refractive index modulation in $1 \%-8 \% \mathrm{Er} / \mathrm{Yb}$ doped fiber to only half of that in undoped fiber. One promising feature of UV-induced Bragg gratings in phosphate fibers is their thermal 
growth of grating reflectivity upon heating at elevated temperatures. Strong gratings with reflectivity of up to $99.9 \%$ have been obtained after the photo-thermal growth. Thermal annealing experiment results demonstrated that the strong growth of the fiber Bragg grating reflectivity observed upon heating at temperatures between $100-250{ }^{\circ} \mathrm{C}$ is directly related to the UV irradiation time, but not to the size of the index modulation of the seed grating or even to the fiber type ( $\mathrm{Er} / \mathrm{Yb}$ doped or undoped). The conditions to reliably obtain final index modulations amplitudes between 5 and $10 \times 10^{-5}$ are given. A tentative model of the photosensitivity is proposed to explain the anomalous but beneficial thermal growth of UV-induced refractive index modulation of FBGs with consideration of the thermal evolution of both average and modulated index change upon heating treatment. Side effects such as ablation and photodarkening were also observed during grating fabrication in phosphate fibers.

The formation of Bragg gratings in phosphate fibers is found to be accompanied by a remarkable photodarkening loss of up to $\sim 1 \mathrm{~dB} / \mathrm{cm}$ across $\mathrm{C}$-band. This finding is very novel as photodarkening losses induced in different kinds of optical fibers were mostly reported to be noticeable at shorter wavelength band $(<1100 \mathrm{~nm})$ while becoming negligible at C-band. It is detrimental to laser efficiency as additional internal cavity losses are introduced in DFB or DBR $\mathrm{Er} / \mathrm{Yb}$ doped fiber lasers based on UV-induced FBGs. A full characterization of the photodarkening effect in phosphate fibers is performed in Chapter 4, by measuring its broadband VIS-NIR absorption spectrum. $\mathrm{Er} / \mathrm{Yb}$ doped and undoped phosphate fibers exhibit the same level of significant UVinduced photodarkening loss, which is associated with the formation of a color center band at $467 \mathrm{~nm}$. A color center species - phosphate oxygen hole center (POHC) is 
suggested to be the origin of photodarkening loss because of their similar characteristic wavelengths of absorption band. Photo-bleaching by a high power supercontinuum source, and thermal-bleaching processes are performed on photodarkened samples. Both bleaching processes are found effective in fully erasing the UV-induced photodarkening loss under condition of not diminishing the grating strength.

The success of direct-writing strong gratings with ArF laser makes it feasible to build short monolithic all-phosphate fiber lasers. A 3.5-cm-long single-wavelength narrow-linewidth distributed feedback fiber laser is implemented in Chapter 5. The DFB grating structure is directly written into the $1 \%-2 \% \mathrm{Er} / \mathrm{Yb}$ doped phosphate glass fibers. A laser signal with a linewidth of $20 \mathrm{MHz}$ and an output power of $27.5 \mathrm{~mW}$ is achieved. The slope efficiency with respect to the absorbed pump power is $17.2 \%$.

A 5-cm-long monolithic dual-wavelength single-longitudinal-mode distributed Bragg reflector all-phosphate fiber laser is demonstrated in Chapter 6. Two strong UVinduced fiber Bragg gratings are directly written in highly $\mathrm{Er} / \mathrm{Yb}$ co-doped phosphate fiber. The separation between gratings is selected as $1 \mathrm{~cm}$ so as to only excite two longitudinal modes in the DBR cavity. By exploiting the spatial hole burning effect and the polarization hole burning effect, stable narrow-linewidth dual-wavelength lasing emission with $38 \mathrm{pm}$ wavelength spacing and a total emitted power of $2.8 \mathrm{~mW}$ is obtained from this DBR fiber laser. A microwave signal at $4.58 \mathrm{GHz}$ is generated by the heterodyne detection of the dual-wavelength laser. 


\subsection{Future work}

This thesis is a starting work on direct-written fiber Bragg gratings in phosphate glass fibers with intense $193 \mathrm{~nm}$ ArF laser irradiation. Future work should be carried out in several research directions as follows.

Although the UV-induced refractive index modulation of $1 \times 10^{-4}$ is achieved, there is still a need for improvement on UV-induced index change of phosphate fibers, considering the low slope efficiency of direct-written grating-based fiber lasers compared to some current all-fiber single-longitudinal-mode lasers. One feasible way of further improving UV-induced index change is to use a longer UV irradiation, or a higher laser pulse intensity, which can be achieved by using a focusing cylindrical lens with a higher magnification factor or removing the $3 \times$ beam expander.

Two fundamental aspects of UV laser-induced modification in phosphate fibers should be also considered in order to achieve larger UV-induced index change. First, it is necessary to identify whether the refractive index modification occurs only in the fiber core region or in both fiber core and cladding regions. This can be checked by measuring the refractive index distribution profile of an exposed fiber with an interferometric fiber profilometer [44]. If a refractive index modification is observed in the cladding region, it would suggest that the cladding region could have a similar UV absorption coefficient to the core region. This absorption in fiber cladding could be a cause for the low photosensitivity of phosphate glass fibers as the UV light has been attenuated by the cladding before it reaches the fiber core.

Second, the ablation effect could also be a factor causing the reduction of the light intensity at the fiber core. As observed in Chapter 3, shallow surface damage on the 
phosphate fiber outer surface was caused by laser ablation during UV irradiation. It is necessary to identify whether the ablation occurs at the front or the rear surface of the optical fiber. If it occurs at the front surface, this damage could scatter or diffract the incident UV light and decrease light intensity at the fiber core. One possible approach to keep the influence of the ablation effect to a low level is to use a more convergent beam, which is usually used in high-intensity fs-laser grating writing setups. By using the focusing cylindrical lens with a higher magnification factor in our grating fabrication setup, the light intensity at the fiber outer surface will decrease and ablation effect will become less severe correspondingly.

The improvement of DFB laser efficiency relies on the optimization of grating reflectivity and the enhancement of grating index modulation. The above proposed research directions should help to achieve higher index modulation in phosphate fibers. A further increase of index modulation can be accomplished with the unique thermal growth feature of phosphate FBGs. For the dual-wavelength SLM DBR phosphate fiber laser, the polarization states of two lasing wavelengths were not perpendicular but at an angle of $70^{\circ}$. The laser stability can be improved with two FBGs written using the same incident direction or perpendicular directions of irradiation beams, which utilizes the polarization hole burning effect to the highest degree to suppress mode competition. 


\section{Publication List}

\section{Publication included in the thesis}

\section{Refereed Journal Papers}

1. L. Xiong, P. Hofmann, A. Schülzgen, N. Peyghambarian, and J. Albert, "Deep UV-induced near-infrared photodarkening of Er/Yb-doped and undoped phosphate fibers," Opt. Lett. 38, 4193-4196 (2013).

2. L. Xiong, P. Hofmann, A. Schülzgen, N. Peyghambarian, and J. Albert, "Short monolithic dual-wavelength single-longitudinal-mode DBR phosphate fiber laser," Appl. Opt. 53, 3848-3853 (2014).

3. L. Xiong, P. Hofmann, A. Schülzgen, N. Peyghambarian, and J. Albert, "Photosensitivity and thermal stability of UV-induced fiber Bragg gratings in phosphate glass fibers," Opt. Mater. Express 4, 1427-1435 (2014).

\section{Conference Papers}

4. L. Xiong, P. Hofmann, A. Schülzgen, N. Peyghambarian, and J. Albert, "Photo-thermal Growth of Unsaturated and Saturated Bragg Gratings in Phosphate Glass Fibers," in Bragg Gratings, Photosensitivity, and Poling in Glass Waveguides (Optical Society of America, 2010), p. BTuB1.

5. L. Xiong, P. Hofmann, A. Schülzgen, N. Peyghambarian, and J. Albert, "A Short Dualwavelength DBR Phosphate Fiber Laser," in CLEO:2011 - Laser Applications to Photonic Applications (OSA, 2011), p. CTuI3.

\section{Publication not included in the thesis}

6. L. Xiong and J. Albert, "Effect of writing beam spatial coherence on fiber Bragg grating modulation contrast and thermal stability," J. Opt. Soc. Am. B 26, 2136-2142 (2009).

7. L.-Y. Shao, L. Xiong, C. Chen, A. Laronche, and J. Albert, "Directional Bend Sensor Based on Re-Grown Tilted Fiber Bragg Grating," J. Light. Technol. 28, 2681-2687 (2010). 
8. C. Shen, L. Xiong, A. Bialiayeu, Y. Zhang, and J. Albert, "Polarization-Resolved Near- and Far-Field Radiation from Near-Infrared Tilted Fiber Bragg Gratings," J. Light. Technol. 32, 2157-2162 (2014).

9. A. Schülzgen, P. Hofmann, L. Li, N. Peyghambarian, L. Xiong, A. Laronche, and J. Albert, "Distributed Feedback Lasers in Phosphate Glass Active Fiber," in Fiber Laser Applications (OSA, 2011), p. FThA5.

10. P. Hofmann, A. Pirson-Chavez, A. Schülzgen, L. Xiong, A. Laronche, J. Albert, and N. Peyghambarian, "Low-noise single frequency all phosphate fiber laser," in Proc. SPIE 8039 , Laser Technology for Defense and Security VII (2011), Vol. 8039, pp. 803911-803916.

11. Y. Zhang, T. Sun, L. Xiong, M. Z. Alam, and J. Albert, "Distributed transverse load sensing with tilted fiber Bragg gratings using optical frequency domain reflectometry," in Proc. SPIE 8421 (2012), p. 84214S. 


\section{Bibliography}

1. M. J. F. Digonnet, Rare-Earth-Doped Fiber Lasers and Amplifiers (Marcel Dekker, 2001).

2. P. Laporta, S. Taccheo, S. Longhi, O. Svelto, and C. Svelto, "Erbium-ytterbium microlasers: optical properties and lasing characteristics," Opt. Mater. 11, 269-288 (1999).

3. B. Hwang, S. Jiang, T. Luo, J. Watson, S. Honkanen, Y. Hu, F. Smektala, J. Lucas, and N. Peyghambarian, "Erbium-doped phosphate glass fibre amplifiers with gain per unit length of $2.1 \mathrm{~dB} / \mathrm{cm}$," Electron. Lett. 35, 1007-1009 (1999).

4. C. Spiegelberg, J. Geng, Y. Hu, Y. Kaneda, S. Jiang, and N. Peyghambarian, "Low-Noise Narrow-Linewidth Fiber Laser at 1550 nm," J. Light. Technol. 22, 57-62 (2004).

5. S. Pissadakis, A. Ikiades, P. Hua, A. Sheridan, and J. Wilkinson, "Photosensitivity of ionexchanged Er-doped phosphate glass using 248nm excimer laser radiation," Opt. Express 12, 3131-3136 (2004).

6. L. Li, M. Morrell, T. Qiu, V. L. Temyanko, A. Schülzgen, A. Mafi, D. Kouznetsov, J. V. Moloney, T. Luo, S. Jiang, and N. Peyghambarian, "Short cladding-pumped Er/Yb phosphate fiber laser with 1.5 W output power," Appl. Phys. Lett. 85, 2721-2723 (2004).

7. T. Qiu, L. Li, A. Schülzgen, V. L. Temyanko, T. Luo, S. Jiang, A. Mafi, J. V. Moloney, and N. Peyghambarian, "Generation of 9.3-W Multimode and 4-W Single-Mode Output From 7-cm Short Fiber Lasers," IEEE Photon. Technol. Lett. 16, 2592-2594 (2004).

8. L. Li, A. Schülzgen, V. L. Temyanko, T. Qiu, M. M. Morrell, Q. Wang, A. Mafi, J. V. Moloney, and N. Peyghambarian, "Short-length microstructured phosphate glass fiber lasers with large mode areas," Opt. Lett. 30, 1141-1143 (2005).

9. T. Qiu, S. Suzuki, A. Schülzgen, L. Li, A. Polynkin, V. Temyanko, J. V Moloney, and N. Peyghambarian, "Generation of watt-level single-longitudinal-mode output from claddingpumped short fiber lasers," Opt. Lett. 30, 2748-2750 (2005).

10. L. Li, A. Schülzgen, V. L. Temyanko, M. M. Morrell, S. Sabet, H. Li, J. V. Moloney, and N. Peyghambarian, "Ultracompact cladding-pumped 35-mm-short fiber laser with 4.7-W single-mode output power," Appl. Phys. Lett. 88, 161106 (2006).

11. A. Schülzgen, L. Li, V. L. Temyanko, S. Suzuki, J. V Moloney, and N. Peyghambarian, "Single-frequency fiber oscillator with watt-level output power using photonic crystal phosphate glass fiber," Opt. Express 14, 7087-7092 (2006).

12. J. Albert, A. Schülzgen, V. L. Temyanko, S. Honkanen, and N. Peyghambarian, "Strong Bragg gratings in phosphate glass single mode fiber," Appl. Phys. Lett. 89, 101127 (2006). 
13. R. M. Rogojan, A. Schülzgen, N. Peyghambarian, A. Laronche, and J. Albert, "Photothermal gratings in $\mathrm{Er}^{3+} / \mathrm{Yb}^{3+}$-doped core phosphate glass single mode fibers," in Bragg Gratings, Photosensitivity, and Poling in Glass Waveguides (Optical Society of America, 2007), p. BTuC3.

14. J. L. Zyskind, V. Mizrahi, D. J. DiGiovanni, and J. W. Sulhoff, "Short single frequency erbium-doped fibre laser," Electron. Lett. 28, 1385-1387 (1992).

15. G. A. Ball, W. W. Morey, G. Hull-Allen, and C. Holton, "Low noise single frequency linear fibre laser," Electron. Lett. 29, 1623-1625 (1993).

16. V. Mizrahi, D. J. DiGiovanni, R. M. Atkins, S. G. Grubb, and J.-M. P. Delavaux, "Stable single-mode erbium fiber-grating laser for digital communication," J. Light. Technol. 11, 2021-2025 (1993).

17. G. A. Ball, C. E. Holton, G. Hull-Allen, and W. W. Morey, "60 mW $1.5 \mu \mathrm{m}$ singlefrequency low-noise fiber laser MOPA," IEEE Photon. Technol. Lett. 6, 192-194 (1994).

18. W. H. Loh and R. I. Laming, "1.55 $\mu \mathrm{m}$ phase-shifted distributed feedback fibre laser," Electron. Lett. 31, 1440-1442 (1995).

19. J. Hübner, P. Varming, M. Sejka, and M. Kristensen, "Distributed feedback $\mathrm{Er}^{3+}$-doped fibre laser," Electron. Lett. 31, 1445-1446 (1995).

20. M. Svalgaard and S. L. Gilbert, "Stability of short, single-mode erbium-doped fiber lasers," Appl. Opt. 36, 4999-5009 (1997).

21. G. Bonfrate, F. Vaninetti, and F. Negrisolo, "Single-frequency MOPA $\mathrm{Er}^{3+} \mathrm{DBR}$ fiber laser for WDM digital telecommunication systems," IEEE Photon. Technol. Lett. 10, 1109-1111 (1998).

22. L. Dong, W. H. Loh, J. E. Caplen, J. D. Minelly, K. Hsu, and L. Reekie, "Efficient singlefrequency fiber lasers with novel photosensitive Er/Yb optical fibers," Opt. Lett. 22, 694696 (1997).

23. W. H. Loh, B. N. Samson, L. Dong, G. J. Cowle, and K. Hsu, "High performance single frequency fiber grating-based erbium/ytterbium-codoped fiber lasers," J. Light. Technol. 16, 114-118 (1998).

24. A. Schülzgen, L. Li, D. Nguyen, C. Spiegelberg, R. M. Rogojan, A. Laronche, J. Albert, and N. Peyghambarian, "Distributed feedback fiber laser pumped by multimode laser diodes," Opt. Lett. 33, 614-616 (2008).

25. L. Li, A. Schülzgen, X. Zhu, J. V. Moloney, J. Albert, and N. Peyghambarian, "1 W tunable dual-wavelength emission from cascaded distributed feedback fiber lasers," Appl. Phys. Lett. 92, 051111 (2008).

26. A. Othonos and K. Kalli, Fiber Bragg Gratings: Fundamentals and Applications in Telecommunications and Sensing (Artech House, 1999). 
27. D. K. Lam and B. K. Garside, "Characterization of single-mode optical fiber filters," Appl. Opt. 20, 440-445 (1981).

28. T. Erdogan, "Fiber grating spectra," J. Light. Technol. 15, 1277-1294 (1997).

29. A. W. Snyder and J. Love, Optical Waveguide Theory (Chapman and Hall, 1983).

30. G. Meltz, W. W. Morey, and W. H. Glenn, "Formation of Bragg gratings in optical fibers by a transverse holographic method," Opt. Lett. 14, 823-825 (1989).

31. K. O. Hill, B. Malo, F. Bilodeau, D. C. Johnson, and J. Albert, "Bragg gratings fabricated in monomode photosensitive optical fiber by UV exposure through a phase mask," Appl. Phys. Lett. 62, 1035-1037 (1993).

32. D. Z. Anderson, V. Mizrahi, T. Erdogan, and A. E. White, "Production of in-fibre gratings using a diffractive optical element," Electron. Lett. 29, 566-568 (1993).

33. R. Kashyap, Fiber Bragg Gratings, 2nd ed. (Academic Press, 2009).

34. K. O. Hill, Y. Fujii, D. C. Johnson, and B. S. Kawasaki, "Photosensitivity in optical fiber waveguides: Application to reflection filter fabrication," Appl. Phys. Lett. 32, 647-649 (1978).

35. D. N. Nikogosyan, "Multi-photon high-excitation-energy approach to fibre grating inscription," Meas. Sci. Technol. 18, R1-R29 (2007).

36. M. Lancry and B. Poumellec, "UV laser processing and multiphoton absorption processes in optical telecommunication fiber materials," Phys. Rep. 523, 207-229 (2013).

37. R. M. Atkins, V. Mizrahi, and T. Erdogan, "248 nm induced vacuum UV spectral changes in optical fibre preform cores: support for a colour centre model of photosensitivity," Electron. Lett. 29, 385-387 (1993).

38. D. P. Hand and P. S. J. Russell, "Photoinduced refractive-index changes in germanosilicate fibers," Opt. Lett. 15, 102-104 (1990).

39. B. Poumellec, I. Riant, P. Niay, P. Bernage, and J. F. Bayon, "UV induced densification during Bragg grating inscription in $\mathrm{Ge}: \mathrm{SiO} 2$ preforms: interferometric microscopy investigations," Opt. Mater. 4, 404-409 (1995).

40. S. Suzuki, A. Schülzgen, S. Sabet, J. V. Moloney, and N. Peyghambarian, "Photosensitivity of Ge-doped phosphate glass to $244 \mathrm{~nm}$ irradiation," Appl. Phys. Lett. 89, 171913 (2006).

41. S. Yliniemi, S. Honkanen, A. Ianoul, A. Laronche, and J. Albert, "Photosensitivity and volume gratings in phosphate glasses for rare-earth-doped ion-exchanged optical waveguide lasers," J. Opt. Soc. Am. B 23, 2470-2478 (2006). 
42. M. Sozzi, A. Rahman, and S. Pissadakis, "Non-monotonous refractive index changes recorded in a phosphate glass optical fibre using 248nm, 500fs laser radiation," Opt. Mater. Express 1, 121-127 (2011).

43. D. Grobnic, S. J. Mihailov, R. B. Walker, C. W. Smelser, C. Lafond, and A. Croteau, "Bragg Gratings Made With a Femtosecond Laser in Heavily Doped Er-Yb Phosphate Glass Fiber," IEEE Photon. Technol. Lett. 19, 943-945 (2007).

44. P. Hofmann, C. Voigtlander, S. Nolte, N. Peyghambarian, and A. Schülzgen, "550-mW Output Power From a Narrow Linewidth All-Phosphate Fiber Laser," J. Light. Technol. 31, 756-760 (2013).

45. P. Dekker, M. Ams, G. D. Marshall, D. J. Little, and M. J. Withford, "Annealing dynamics of waveguide Bragg gratings: evidence of femtosecond laser induced colour centres," Opt. Express 18, 3274-3283 (2010).

46. L. B. Fletcher, J. J. Witcher, N. Troy, S. T. Reis, R. K. Brow, and D. M. Krol, "Direct femtosecond laser waveguide writing inside zinc phosphate glass," Opt. Express 19, 7929-7936 (2011).

47. L. B. Fletcher, J. J. Witcher, N. Troy, S. T. Reis, R. K. Brow, R. M. Vazquez, R. Osellame, and D. M. Krol, "Femtosecond laser writing of waveguides in zinc phosphate glasses," Opt. Mater. Express 1, 845-855 (2011).

48. D. J. Little, M. Ams, P. Dekker, G. D. Marshall, and M. J. Withford, "Mechanism of femtosecond-laser induced refractive index change in phosphate glass under a low repetition-rate regime," J. Appl. Phys. 108, 033110 (2010).

49. S. Gross, M. Ams, G. Palmer, C. T. Miese, R. J. Williams, G. D. Marshall, A. Fuerbach, D. G. Lancaster, H. Ebendorff-Heidepriem, and M. J. Withford, "Ultrafast Laser Inscription in Soft Glasses: A Comparative Study of Athermal and Thermal Processing Regimes for Guided Wave Optics," Int. J. Appl. Glas. Sci. 3, 332-348 (2012).

50. K. Seneschal, F. Smektala, B. Bureau, M. Le Floch, S. Jiang, T. Luo, J. Lucas, and N. Peyghambarian, "Properties and structure of high erbium doped phosphate glass for short optical fibers amplifiers," Mater. Res. Bull. 40, 1433-1442 (2005).

51. R. K. Brow, "Review: the structure of simple phosphate glasses," J. Non. Cryst. Solids 263-264, 1-28 (2000).

52. U. Natura and D. Ehrt, "Generation and healing behavior of radiation-induced optical absorption in fluoride phosphate glasses: The dependence on UV radiation sources and temperature," Nucl. Instrum. Meth. B 174, 143-150 (2001).

53. U. Natura, T. Feurer, and D. Ehrt, "Kinetics of UV laser radiation defects in high performance glasses," Nucl. Instrum. Meth. B 166-167, 470-475 (2000). 
54. D. Ehrt, P. Ebeling, and U. Natura, "UV Transmission and radiation-induced defects in phosphate and fluoride-phosphate glasses," J. Non. Cryst. Solids 263-264, 240-250 (2000).

55. J. W. Chan, T. Huser, J. S. Hayden, S. H. Risbud, and D. M. Krol, "Fluorescence Spectroscopy of Color Centers Generated in Phosphate Glasses after Exposure to Femtosecond Laser Pulses," J. Am. Ceram. Soc. 85, 1037-1040 (2004).

56. P. Ebeling, D. Ehrt, and M. Friedrich, "X-ray induced effects in phosphate glasses," Opt. Mater. 20, 101-111 (2002).

57. I. Michelakaki and S. Pissadakis, "Atypical behaviour of the surface hardness and the elastic modulus of a phosphate glass matrix under 193 nm laser irradiation," Appl. Phys. A 95, 453-456 (2008).

58. L. B. Fletcher, J. J. Witcher, W. B. Reichman, A. Arai, J. Bovatsek, and D. M. Krol, "Changes to the network structure of $\mathrm{Er}-\mathrm{Yb}$ doped phosphate glass induced by femtosecond laser pulses," J. Appl. Phys. 106, 083107 (2009).

59. L. Xiong, P. Hofmann, A. Schülzgen, N. Peyghambarian, and J. Albert, "Photosensitivity and thermal stability of UV-induced fiber Bragg gratings in phosphate glass fibers," Opt. Mater. Express 4, 1427-1435 (2014).

60. L. Xiong, P. Hofmann, A. Schülzgen, N. Peyghambarian, and J. Albert, "Photo-thermal Growth of Unsaturated and Saturated Bragg Gratings in Phosphate Glass Fibers," in Bragg Gratings, Photosensitivity, and Poling in Glass Waveguides (Optical Society of America, 2010), p. BTuB1.

61. P. E. Dyer, R. J. Farley, R. Giedl, and D. M. Karnakis, "Excimer laser ablation of polymers and glasses for grating fabrication," Appl. Surf. Sci. 96-98, 537-549 (1996).

62. J. D. Musgraves, K. Richardson, and H. Jain, "Laser-induced structural modification, its mechanisms, and applications in glassy optical materials," Opt. Mater. Express 1, 921-935 (2011).

63. A. Hidayat, Q. Wang, P. Niay, M. Douay, B. Poumellec, F. Kherbouche, and I. Riant, "Temperature-Induced Reversible Changes in the Spectral Characteristics of Fiber Bragg Gratings," Appl. Opt. 40, 2632 (2001).

64. S. Bandyopadhyay, J. Canning, M. Stevenson, and K. Cook, "Ultrahigh-temperature regenerated gratings in boron-codoped germanosilicate optical fiber using $193 \mathrm{~nm}$," Opt. Lett. 33, 1917-1919 (2008).

65. L. Xiong, P. Hofmann, A. Schülzgen, N. Peyghambarian, and J. Albert, "Deep UVinduced near-infrared photodarkening of $\mathrm{Er} / \mathrm{Yb}$-doped and undoped phosphate fibers," Opt. Lett. 38, 4193-4196 (2013).

66. J. Albert, "Permanent Photoinduced Changes for Bragg Gratings in Silicate Glass Waveguides and Fibers," MRS Bull. 23, 36-42 (1998). 
67. Y. W. Lee, S. Sinha, M. J. F. Digonnet, R. L. Byer, and S. Jiang, "Measurement of high photodarkening resistance in heavily $\mathrm{Yb}^{3+}$-doped phosphate fibres," Electron. Lett. 44, 14 16 (2008).

68. M. M. Broer, D. M. Krol, and D. J. DiGiovanni, "Highly nonlinear near-resonant photodarkening in a thulium-doped aluminosilicate glass fiber," Opt. Lett. 18, 799-801 (1993).

69. G. R. Atkins and A. L. G. Carter, "Photodarkening in $\mathrm{Tb}^{3+}$-doped phosphosilicate and germanosilicate optical fibers," Opt. Lett. 19, 874-876 (1994).

70. J. J. Koponen, M. J. Söderlund, H. J. Hoffman, and S. K. T. Tammela, "Measuring photodarkening from single-mode ytterbium doped silica fibers," Opt. Express 14, 11539 11544 (2006).

71. J. Koponen, M. Söderlund, H. J. Hoffman, D. A. V. Kliner, J. P. Koplow, and M. Hotoleanu, "Photodarkening rate in Yb-doped silica fibers," Appl. Opt. 47, 1247-1256 (2008).

72. M. J. Söderlund, J. J. Montiel i Ponsoda, J. P. Koplow, and S. Honkanen, "Thermal bleaching of photodarkening-induced loss in ytterbium-doped fibers," Opt. Lett. 34, 2637 2639 (2009).

73. C. Ye, J. J. Montiel i Ponsoda, A. Tervonen, and S. Honkanen, "Refractive index change in ytterbium-doped fibers induced by photodarkening and thermal bleaching," Appl. Opt. 49, 5799-5805 (2010).

74. J. Canning, A. L. G. Carter, and M. G. Sceats, "Correlation between photodarkening and index change during $193 \mathrm{~nm}$ irradiation of germanosilicate and phosphosilicate fibers," J. Light. Technol. 15, 1348-1356 (1997).

75. S. Yoo, C. Basu, A. J. Boyland, C. Sones, J. Nilsson, J. K. Sahu, and D. Payne, "Photodarkening in Yb-doped aluminosilicate fibers induced by $488 \mathrm{~nm}$ irradiation," Opt. Lett. 32, 1626-1628 (2007).

76. M. Engholm, L. Norin, and D. Aberg, "Strong UV absorption and visible luminescence in ytterbium-doped aluminosilicate glass under UV excitation," Opt. Lett. 32, 3352-3354 (2007).

77. R. Peretti, C. Gonnet, and A.-M. Jurdyc, "Revisiting literature observations on photodarkening in $\mathrm{Yb}^{3+}$ doped fiber considering the possible presence of Tm impurities," J. Appl. Phys. 112, 093511 (2012).

78. J. Fiebrandt, S. Jetschke, M. Leich, M. Rothhardt, and H. Bartelt, "UV-induced photodarkening and photobleaching in UV-femtosecond-pulse-written fibre Bragg gratings," Laser Phys. Lett. 10, 085102 (2013).

79. Q. Zhou, L. Xu, L. Liu, W. Wang, C. Zhu, and F. Gan, "Study on the laser-induced darkening in Nd-doped laser glasses," Opt. Mater. 25, 313-319 (2004). 
80. M. Leich, J. Fiebrandt, A. Schwuchow, S. Unger, S. Jetschke, and H. Bartelt, "Femtosecond pulse-induced fiber Bragg gratings for in-core temperature measurement in optically pumped Yb-doped silica fibers," Opt. Commun. 285, 4387-4390 (2012).

81. L. Xiong, P. Hofmann, A. Schülzgen, N. Peyghambarian, and J. Albert, "A Short Dualwavelength DBR Phosphate Fiber Laser," in CLEO:2011 - Laser Applications to Photonic Applications (OSA, 2011), p. CTuI3.

82. L. Xiong, P. Hofmann, A. Schülzgen, N. Peyghambarian, and J. Albert, "Short monolithic dual-wavelength single-longitudinal-mode DBR phosphate fiber laser," Appl. Opt. 53, 3848-3853 (2014).

83. B. Hwang, S. Jiang, T. Luo, J. Watson, G. Sorbello, and N. Peyghambarian, "Cooperative upconversion and energy transfer of new high $\mathrm{Er}^{3+}$ - and $\mathrm{Yb}^{3+}-\mathrm{Er}^{3+}$-doped phosphate glasses," J. Opt. Soc. Am. B 17, 833 (2000).

84. X. Zhu, A. Schülzgen, H. Li, L. Li, Q. Wang, S. Suzuki, V. L. Temyanko, J. V Moloney, and N. Peyghambarian, "Single-transverse-mode output from a fiber laser based on multimode interference," Opt. Lett. 33, 908-910 (2008).

85. S. H. Xu, Z. M. Yang, T. Liu, W. N. Zhang, Z. M. Feng, Q. Y. Zhang, and Z. H. Jiang, "An efficient compact $300 \mathrm{~mW}$ narrow-linewidth single frequency fiber laser at $1.5 \mu \mathrm{m}$," Opt. Express 18, 1249-1254 (2010).

86. P. Hofmann, A. Pirson-Chavez, A. Schülzgen, L. Xiong, A. Laronche, J. Albert, and N. Peyghambarian, "Low-noise single frequency all phosphate fiber laser," in Proc. SPIE 8039, Laser Technology for Defense and Security VII (2011), Vol. 8039, pp. 803911803916.

87. S. Pradhan, G. E. Town, and K. J. Grant, "Dual-wavelength DBR fiber laser," IEEE Photon. Technol. Lett. 18, 1741-1743 (2006).

88. Y. Yao, X. Chen, Y. Dai, and S. Xie, "Dual-wavelength erbium-doped fiber laser with a simple linear cavity and its application in microwave generation," IEEE Photon. Technol. Lett. 18, 187-189 (2006).

89. Y. Dai, X. Chen, J. Sun, Y. Yao, and S. Xie, "Dual-Wavelength DFB Fiber Laser Based on a Chirped Structure and the Equivalent Phase Shift Method," IEEE Photon. Technol. Lett. 18, 1964-1966 (2006).

90. G. E. Villanueva, P. Perez-Millan, J. Palaci, J. L. Cruz, M. V. Andres, and J. Marti, "DualWavelength DFB Erbium-Doped Fiber Laser With Tunable Wavelength Spacing," IEEE Photon. Technol. Lett. 22, 254-256 (2010).

91. L. Sun, X. Feng, W. Zhang, L. Xiong, Y. Liu, G. Kai, S. Yuan, and X. Dong, "Beating frequency tunable dual-wavelength erbium-doped fiber laser with one fiber Bragg grating," IEEE Photon. Technol. Lett. 16, 1453-1455 (2004). 
92. S. Feng, O. Xu, S. Lu, X. Mao, T. Ning, and S. Jian, "Single-polarization, switchable dualwavelength erbium-doped fiber laser with two polarization-maintaining fiber Bragg gratings," Opt. Express 16, 11830-11835 (2008).

93. W. Liu, M. Jiang, D. Chen, and S. He, "Dual-Wavelength Single-Longitudinal-Mode Polarization-Maintaining Fiber Laser and Its Application in Microwave Generation," J. Light. Technol. 27, 4455-4459 (2009).

94. P. Rugeland, Z. Yu, O. Tarasenko, G. Tengstrand, and W. Margulis, "Tunable Photonic Microwave Generation Based on a Novel Dual-Polarization Fiber Laser Cavity," IEEE Photon. Technol. Lett. 23, 1878-1880 (2011).

95. X. He, X. Fang, C. Liao, D. N. Wang, and J. Sun, "A tunable and switchable singlelongitudinal-mode dual-wavelength fiber laser with a simple linear cavity," Opt. Express 17, 21773-21781 (2009).

96. B. Lin, S. Chuan Tjin, M. Jiang, and P. Shum, "Tunable microwave generation based on a dual-wavelength fiber laser with an inverse-Gaussian apodized fiber Bragg grating," Appl. Opt. 50, 4912-4916 (2011).

97. S. Pan, X. Zhao, and C. Lou, "Switchable single-longitudinal-mode dual-wavelength erbium-doped fiber ring laser incorporating a semiconductor optical amplifier," Opt. Lett. 33, 764-766 (2008).

98. B. Lin, S. C. Tjin, H. Zhang, D. Tang, J. Hao, B. Dong, and S. Liang, "Switchable dualwavelength single-longitudinal-mode erbium-doped fiber laser using an inverse-Gaussian apodized fiber Bragg grating filter and a low-gain semiconductor optical amplifier," Appl. Opt. 49, 6855-6860 (2010).

99. M. Tang, H. Minamide, Y. Wang, T. Notake, S. Ohno, and H. Ito, "Tunable Terahertzwave generation from DAST crystal pumped by a monolithic dual-wavelength fiber laser," Opt. Express 19, 779-786 (2011).

100. M. A. Ummy, N. Madamopoulos, M. Razani, A. Hossain, and R. Dorsinville, "Switchable dual-wavelength SOA-based fiber laser with continuous tunability over the C-band at room-temperature," Opt. Express 20, 23367-23373 (2012).

101. Y. Liu, L. Wei, and J. W. Y. Lit, "Transmission loss of phase-shifted fiber Bragg gratings in lossy materials: a theoretical and experimental investigation," Appl. Opt. 46, 67706773 (2007).

102. Y. O. Barmenkov, D. Zalvidea, S. Torres-Peiró, J. L. Cruz, and M. V Andrés, "Effective length of short Fabry-Perot cavity formed by uniform fiber Bragg gratings," Opt. Express 14, 6394-6399 (2006). 